Cochrane Database of Systematic Reviews

\title{
Prophylactic plasma transfusion for patients without inherited bleeding disorders or anticoagulant use undergoing non-cardiac surgery or invasive procedures (Review)
}

Huber J, Stanworth SJ, Doree C, Fortin PM, Trivella M, Brunskill SJ, Hopewell S, Wilkinson KL, Estcourt LJ

Huber J, Stanworth SJ, Doree C, Fortin PM, Trivella M, Brunskill SJ, Hopewell S, Wilkinson KL, Estcourt LJ.

Prophylactic plasma transfusion for patients without inherited bleeding disorders or anticoagulant use undergoing non-cardiac surgery or invasive procedures.

Cochrane Database of Systematic Reviews 2019, Issue 11. Art. No.: CD012745.

DOI: 10.1002/14651858.CD012745.pub2. 
TABLE OF CONTENTS

HEADER 1

ABSTRACT

PLAIN LANGUAGE SUMMARY

SUMMARY OF FINDINGS

BACKGROUND

OBJECTIVES

METHODS

RESULTS

Figure 1.

Figure 2.

Figure 3.

DISCUSSION

AUTHORS' CONCLUSIONS

ACKNOWLEDGEMENTS

REFERENCES

CHARACTERISTICS OF STUDIES

DATA AND ANALYSES

Analysis 1.1. Comparison 1 Prophylactic plasma transfusion before surgery versus no prophylactic plasma transfusion before surgery (colloid, crystalloid, placebo, or no treatment), Outcome 1 All-cause mortality.

Analysis 1.2. Comparison 1 Prophylactic plasma transfusion before surgery versus no prophylactic plasma transfusion before surgery (colloid, crystalloid, placebo, or no treatment), Outcome 2 Major bleeding.

Analysis 2.1. Comparison 2 Prophylactic plasma transfusion before surgery versus alternative haemostatic agents, Outcome 1 Serious adverse event due to plasma transfusion.

Analysis 2.2. Comparison 2 Prophylactic plasma transfusion before surgery versus alternative haemostatic agents, Outcome 2 Coagulation test abnormalities.

Analysis 3.1. Comparison 3 Thromboelastography threshold versus standard of care (laboratory parameters), Outcome 1 Allcause mortality.

Analysis 3.2. Comparison 3 Thromboelastography threshold versus standard of care (laboratory parameters), Outcome 2 Major bleeding.

Analysis 3.3. Comparison 3 Thromboelastography threshold versus standard of care (laboratory parameters), Outcome 3 Number of individuals requiring a transfusion.

Analysis 3.4. Comparison 3 Thromboelastography threshold versus standard of care (laboratory parameters), Outcome 4 Serious adverse event due to plasma transfusion.

ADDITIONAL TABLES

APPENDICES

CONTRIBUTIONS OF AUTHORS

DECLARATIONS OF INTEREST

SOURCES OF SUPPORT

DIFFERENCES BETWEEN PROTOCOL AND REVIEW

INDEX TERMS 
[Intervention Review]

\section{Prophylactic plasma transfusion for patients without inherited bleeding disorders or anticoagulant use undergoing non-cardiac surgery or invasive procedures}

Jonathan Huber ${ }^{1}$, Simon J Stanworth², Carolyn Doree ${ }^{3}$, Patricia M Fortin 4 , Marialena Trivella 5 , Susan J Brunskill33, Sally Hopewell6, Kirstin L Wilkinson? ${ }^{7}$ Lise J Estcourt8

1Shackleton Department of Anaesthesia, University Hospital Southampton NHS Foundation Trust, Southampton, UK. ${ }^{2 N a t i o n a l ~ I n s t i t u t e ~}$ for Health Research (NIHR) Oxford Biomedical Research Centre, Oxford University Hospitals NHS Foundation Trust and University of Oxford, Oxford, UK. ${ }^{3}$ Systematic Review Initiative, NHS Blood and Transplant, Oxford, UK. ${ }^{4}$ Sechelt, Canada. ${ }^{5}$ Centre for Statistics in Medicine, University of Oxford, Oxford, UK. ${ }^{6}$ Nuffield Department of Orthopaedics, Rheumatology and Musculoskeletal Sciences (NDORMS), University of Oxford, Oxford, UK. 7 Paediatric and Adult Cardiothoracic Anaesthesia, Southampton University NHS Hospital, Southampton, UK. ${ }^{8}$ Haematology/Transfusion Medicine, NHS Blood and Transplant, Oxford, UK

Contact address: Lise J Estcourt, Haematology/Transfusion Medicine, NHS Blood and Transplant, Level 2, John Radcliffe Hospital, Headington, Oxford, OX3 9BQ, UK. lise.estcourt@nhsbt.nhs.uk, lise.estcourt@ndcls.ox.ac.uk.

Editorial group: Cochrane Haematology Group.

Publication status and date: New, published in Issue 11, 2019.

Citation: Huber J, Stanworth SJ, Doree C, Fortin PM, Trivella M, Brunskill SJ, Hopewell S, Wilkinson KL, Estcourt LJ. Prophylactic plasma transfusion for patients without inherited bleeding disorders or anticoagulant use undergoing non-cardiac surgery or invasive procedures. Cochrane Database of Systematic Reviews 2019, Issue 11. Art. No.: CD012745. DOI: 10.1002/14651858.CD012745.pub2.

Copyright () 2019 The Cochrane Collaboration. Published by John Wiley \& Sons, Ltd.

\section{A B S T R A C T}

\section{Background}

In the absence of bleeding, plasma is commonly transfused to people prophylactically to prevent bleeding. In this context, it is transfused before operative or invasive procedures (such as liver biopsy or chest drainage tube insertion) in those considered at increased risk of bleeding, typically defined by abnormalities of laboratory tests of coagulation. As plasma contains procoagulant factors, plasma transfusion may reduce perioperative bleeding risk. This outcome has clinical importance given that perioperative bleeding and blood transfusion have been associated with increased morbidity and mortality. Plasma is expensive, and some countries have experienced issues with blood product shortages, donor pool reliability, and incomplete screening for transmissible infections. Thus, although the benefit of prophylactic plasma transfusion has not been well established, plasma transfusion does carry potentially life-threatening risks.

\section{Objectives}

To determine the clinical effectiveness and safety of prophylactic plasma transfusion for people with coagulation test abnormalities (in the absence of inherited bleeding disorders or use of anticoagulant medication) requiring non-cardiac surgery or invasive procedures.

\section{Search methods}

We searched for randomised controlled trials (RCTs), without language or publication status restrictions in: Cochrane Central Register of Controlled Trials (CENTRAL; 2017 Issue 7); Ovid MEDLINE (from 1946); Ovid Embase (from 1974); Cumulative Index to Nursing and Allied Health Literature (CINAHL; EBSCOHost) (from 1937); PubMed (e-publications and in-process citations ahead of print only); Transfusion Evidence Library (from 1950); Latin American Caribbean Health Sciences Literature (LILACS) (from 1982); Web of Science: Conference Proceedings Citation Index-Science (CPCI-S) (Thomson Reuters, from 1990); ClinicalTrials.gov; and World Health Organization (WHO) International Clinical Trials Registry Search Platform (ICTRP) to 28 January 2019. 


\section{Selection criteria}

We included RCTs comparing: prophylactic plasma transfusion to placebo, intravenous fluid, or no intervention; prophylactic plasma transfusion to alternative pro-haemostatic agents; or different haemostatic thresholds for prophylactic plasma transfusion. We included participants of any age, and we excluded trials incorporating individuals with previous active bleeding, with inherited bleeding disorders, or taking anticoagulant medication before enrolment.

\section{Data collection and analysis}

We used standard methodological procedures expected by Cochrane.

\section{Main results}

We included five trials in this review, all were conducted in high-income countries. Three additional trials are ongoing.

One trial compared fresh frozen plasma (FFP) transfusion with no transfusion given. One trial compared FFP or platelet transfusion or both with neither FFP nor platelet transfusion given. One trial compared FFP transfusion with administration of alternative prohaemostatic agents (factors II, IX, and X followed by VII). One trial compared the use of different transfusion triggers using the international normalised ratio measurement. One trial compared the use of a thromboelastographic-guided transfusion trigger using standard laboratory measurements of coagulation.

Four trials enrolled only adults, whereas the fifth trial did not specify participant age. Four trials included only minor procedures that could be performed by the bedside. Only one trial included some participants undergoing major surgical operations. Two trials included only participants in intensive care. Two trials included only participants with liver disease.

Three trials did not recruit sufficient participants to meet their pre-calculated sample size. Overall, the quality of evidence was low to very low across different outcomes according to GRADE methodology, due to risk of bias, indirectness, and imprecision.

One trial was stopped after recruiting two participants, therefore this review's findings are based on the remaining four trials (234 participants).

When plasma transfusion was compared with no transfusion given, we are very uncertain whether there was a difference in 30-day mortality ( 1 trial comparing FFP or platelet transfusion or both with neither FFP nor platelet transfusion, 72 participants; risk ratio (RR) 0.38 , 95\% confidence interval (CI) 0.13 to 1.10 ; very low-quality evidence).

We are very uncertain whether there was a difference in major bleeding within 24 hours (1 trial comparing FFP transfusion vs no transfusion, 76 participants; RR $0.33,95 \% \mathrm{Cl} 0.01$ to 7.93 ; very low-quality evidence; 1 trial comparing FFP or platelet transfusion or both with neither FFP nor platelet transfusion, 72 participants; RR 1.59, 95\% Cl 0.28 to 8.93 ; very low-quality evidence).

We are very uncertain whether there was a difference in the number of blood product transfusions per person ( 1 trial, 76 participants; study authors reported no difference; very low-quality evidence) or in the number of people requiring transfusion ( 1 trial comparing FFP or platelet transfusion or both with neither FFP nor platelet transfusion, 72 participants; study authors reported no blood transfusion given; very low-quality evidence) or in the risk of transfusion-related adverse events (acute lung injury) (1 trial, 76 participants; study authors reported no difference; very low-quality evidence).

When plasma transfusion was compared with other pro-haemostatic agents, we are very uncertain whether there was a difference in major bleeding (1 trial; 21 participants; no events; very low-quality evidence) or in transfusion-related adverse events (febrile or allergic reactions) (1 trial, 21 participants; RR 9.82, 95\% Cl 0.59 to 162.24 ; very low-quality evidence).

When different triggers for FFP transfusion were compared, the number of people requiring transfusion may have been reduced (for overall blood products) when a thromboelastographic-guided transfusion trigger was compared with standard laboratory tests (1 trial, 60 participants; RR $0.18,95 \% \mathrm{Cl} 0.08$ to 0.39 ; low-quality evidence). We are very uncertain whether there was a difference in major bleeding (1 trial, 60 participants; RR $0.33,95 \% \mathrm{Cl} 0.01$ to 7.87 ; very low-quality evidence) or in transfusion-related adverse events (allergic reactions) (1 trial; 60 participants; RR $0.33,95 \% \mathrm{Cl} 0.01$ to 7.87 ; very low-quality evidence).

Only one trial reported 30-day mortality. No trials reported procedure-related harmful events (excluding bleeding) or quality of life.

\section{Authors' conclusions}

Review findings show uncertainty for the utility and safety of prophylactic FFP use. This is due to predominantly very low-quality evidence that is available for its use over a range of clinically important outcomes, together with lack of confidence in the wider applicability of study findings, given the paucity or absence of study data in settings such as major body cavity surgery, extensive soft tissue surgery, orthopaedic surgery, or neurosurgery. Therefore, from the limited RCT evidence, we can neither support nor oppose the use of prophylactic FFP in clinical practice. 


\section{PLAIN LANGUAGE SUMMARY}

\section{Plasma transfusions before major surgery (other than heart surgery) or invasive procedures, to prevent bleeding}

\section{Review question}

Human plasma, a blood component, is often transfused to people before undergoing surgery or other procedures (such as inserting a chest drainage tube) when blood tests show that their blood may not clot adequately. Aims of this review were to assess how effective this practice is in reducing subsequent bleeding or need for blood transfusion, and whether this approach increases risk of death or other significantly harmful effects. The review excluded people with inherited bleeding disorders and those taking medication that reduces their blood's clotting ability.

\section{Background}

Human plasma is obtained from blood donors. It contains many factors that help blood to clot. Although plasma may be transfused to people based on blood tests suggesting that their blood may not clot adequately, these tests have limitations. A person's blood may clot adequately despite abnormal test results. Abnormal test results also do not clearly predict those people who will go on to bleed. Furthermore, plasma transfusion corrects abnormal blood tests to varying degrees.

Plasma is also expensive, and some countries have issues with blood product shortages, donor reliability, and incomplete screening for infections that could be transmitted through blood product transfusion. Given the potential for life-threatening complications from plasma transfusion, its use in this setting carries risk of harm without clear evidence of benefit.

\section{Study characteristics}

We included five trials which were all conducted in high-income countries.

Our search is current up until 28 January 2019. One trial compared plasma transfusion with no transfusion given. Another trial compared plasma or platelet transfusion or both with neither plasma nor platelet transfusion given. One trial compared plasma transfusion with alternative products given to help blood clot. Another trial compared different blood tests to trigger a plasma transfusion, and still another trial compared different transfusion triggers using the same blood test.

Four trials involved adult participants over 18 years old, and the fifth trial did not specify age of participants. In four trials, participants underwent bedside procedures. Only one trial involved some participants undergoing major surgical operations. Two trials included only participants in intensive care, and two trials included only participants with liver disease.

One trial recruited only two participants. Therefore review results include the remaining four trials, incorporating 234 participants. Three further trials are ongoing.

\section{Key results}

When plasma transfusion was compared with no transfusion given, we are very uncertain whether there was a difference in major bleeding, number of blood transfusions per participant, or harmful effects from the transfusion ( 1 trial; very low-quality evidence). When plasma or platelet transfusion or both were compared with neither plasma nor platelet transfusion, we are very uncertain whether there was a difference in mortality within 30 days, or in the number of individuals requiring a transfusion (1 trial; very low-quality evidence).

When plasma transfusion was compared with other haemostatic agents, we are very uncertain whether there was a difference in major bleeding or in harmful effects from the transfusion (1 trial; very low-quality evidence).

When different triggers for plasma transfusion were compared ( 1 trial; 60 participants), we are very uncertain whether there was a difference in major bleeding or in harmful effects from the transfusion due to very low-quality evidence for these outcomes. The number of people requiring blood products may have been reduced overall, although this is based on low-quality evidence.

No trials reported procedure-related harmful events or quality of life as an outcome.

\section{Quality of the evidence}

The overall quality of the evidence was predominantly very low over a range of clinically important outcomes due to combinations of issues within the studies, such as potential for bias, limited clinical settings, and imprecise estimates of intervention effects.

\section{Authors' conclusions}

We are very uncertain of the effectiveness and safety of the use of plasma in non-cardiac operations or invasive procedures due to very low-quality evidence. Furthermore, as trials do not cover a wide range of surgical contexts, our confidence in applying study results to the wider surgical setting is limited. Overall limited evidence for the utility of plasma transfused to people within this context is of insufficient quality to support or oppose its use.

Prophylactic plasma transfusion for patients without inherited bleeding disorders or anticoagulant use undergoing non-cardiac surgery 
SUMMARY OF FINDINGS

Summary of findings for the main comparison. Prophylactic plasma transfusion before surgery/invasive procedures compared to no prophylactic plasma transfusion before surgery/invasive procedures (colloid, crystalloid, placebo, or no treatment) for patients undergoing non-cardiac surgery or invasive procedures

Prophylactic plasma transfusion before surgery/invasive procedures compared to no prophylactic plasma transfusion before surgery/invasive procedures (colloid, crystalloid, placebo, or no treatment) for patients undergoing non-cardiac surgery or invasive procedures

Patient or population: patients undergoing non-cardiac surgery or invasive procedures

Setting: individuals in ICU undergoing invasive procedures. Studies conducted in The Netherlands

Intervention: prophylactic plasma transfusion before surgery/invasive procedures

Comparison: no prophylactic plasma transfusion before surgery/invasive procedures (colloid, crystalloid, placebo, or no treatment)

$\begin{array}{lll}\text { Outcomes } & \text { Relative effect Anticipated absolute effects }{ }^{\star}\left(95 \% C_{i}\right)\end{array}$

$(95 \% \mathrm{Cl})$

Without prophylactic

Without prophylactic
plasma transfusion be-

fore surgery/invasive

procedures dures

With prophylactic plas-

ma transfusion before

surgery/invasive proce-

All-cause mortality up to $\mathbf{3 0}$ days

№ of participants: 72

Study population

RR 0.38

dures

(1 RCT)

(0.13 to 1.10$)$

297 per 1000

113 per 1000
(39 to 327 )

Certainty of

the evidence

(GRADE)

Major bleeding within 24 hours

№ of participants: 148

(2 RCTs)

Two studies; reported separatelyc

$\oplus \ominus \ominus \ominus$

FFP group events 0/38; no FFP group events 1/38; RR 0.33, 95\% Cl 0.01 to 7.93 (Müller 2015)

Very lowb,d,e

FFP or platelets or both group events $3 / 35$; no transfusion group events $2 / 37$; RR $1.59,95 \% \mathrm{Cl} 0.28$ to 8.93 (Veelo 2012)

Number of transfusions per participant within

7 days

Study authors reported no difference ("P $=0.91$ (RBC), $P=0.06$ (FFP), $P=0.43$ (PLT)") between the 2

$\oplus \odot \odot \ominus$

№ of participants: 76

Study authors reported no difference ("P $=0.91$ (RBC
groups (76 participants; Müller 2015). See Table 1

Very low d,f,g

(1 RCT)

Number of individuals requiring a transfusion

within 7 days

Study authors reported "no... transfusion of packed red cells for blood loss during or after [the proce-

dure]" (72 participants; Veelo 2012)

$\oplus \odot \odot \ominus$

№ of participants: 72

(1 RCT) 
Intervention: prophylactic plasma transfusion before surgery/invasive procedures Comparison: alternative haemostatic agents

\begin{tabular}{|c|c|c|c|c|c|}
\hline \multirow[t]{2}{*}{ Outcomes } & \multirow{2}{*}{$\begin{array}{l}\text { Relative ef- } \\
\text { fect } \\
(95 \% \mathrm{CI})\end{array}$} & \multicolumn{3}{|c|}{ Anticipated absolute effects ${ }^{\star}(95 \% \mathrm{Cl})$} & \multirow{2}{*}{$\begin{array}{l}\text { Certainty of } \\
\text { the evidence } \\
\text { (GRADE) }\end{array}$} \\
\hline & & $\begin{array}{l}\text { Without prophylac- } \\
\text { tic plasma transfu- } \\
\text { sion before surgery/ } \\
\text { invasive procedures }\end{array}$ & $\begin{array}{l}\text { With prophylactic } \\
\text { plasma transfusion } \\
\text { before surgery/inva- } \\
\text { sive procedures }\end{array}$ & Difference & \\
\hline All-cause mortality up to $\mathbf{3 0}$ days - not reported & - & - & - & & - \\
\hline $\begin{array}{l}\text { Number of transfusions per participant within } 7 \text { days - not } \\
\text { reported }\end{array}$ & - & - & - & & - \\
\hline $\begin{array}{l}\text { Number of individuals requiring a transfusion within } 7 \text { days } \\
\text { - not reported }\end{array}$ & - & - & - & & - \\
\hline \multirow{6}{*}{$\begin{array}{l}\text { Serious adverse events measured by plasma transfusion-re- } \\
\text { lated complications within } 24 \text { hours } \\
\text { № of participants: } 21 \\
\text { (1 RCT) }\end{array}$} & \multirow{6}{*}{$\begin{array}{l}\text { RR } 9.82 \\
(0.59 \text { to } \\
162.24)\end{array}$} & \multicolumn{3}{|l|}{ Low } & \multirow{6}{*}{$\begin{array}{l}\oplus \ominus \ominus \ominus \\
\text { Very lowd,e }\end{array}$} \\
\hline & & 10 per $1000 c$ & 98 per 1000 & 88 more per 1000 & \\
\hline & & & (6 to 1000$)$ & $\begin{array}{l}\text { (4 fewer to } 1612 \\
\text { more) }\end{array}$ & \\
\hline & & \multicolumn{3}{|l|}{ High } & \\
\hline & & 100 per $1000^{c}$ & 982 per 1000 & 882 more per 1000 & \\
\hline & & & (59 to 1000$)$ & $\begin{array}{l}\text { ( } 41 \text { fewer to } 16124 \\
\text { more) }\end{array}$ & \\
\hline $\begin{array}{l}\text { Serious adverse events measured by surgery or proce- } \\
\text { dure-related complications within } \mathbf{3 0} \text { days - not reported }\end{array}$ & - & - & - & & - \\
\hline Quality of life - not reported & - & - & - & & - \\
\hline
\end{tabular}

${ }^{\star}$ The risk in the intervention group (and its $95 \%$ confidence interval) is based on the assumed risk in the comparison group and the relative effect of the intervention (and its $95 \% \mathrm{Cl}$ ). 


\section{GRADE Working Group grades of evidence.}

High certainty: we are very confident that the true effect lies close to that of the estimate of the effect.

Moderate certainty: we are moderately confident in the effect estimate: the true effect is likely to be close to the estimate of the effect, but there is a possibility that it is substantially different.

Low certainty: our confidence in the effect estimate is limited: the true effect may be substantially different from the estimate of the effect.

Very low certainty: we have very little confidence in the effect estimate: the true effect is likely to be substantially different from the estimate of effect.

aDowngraded two points due to indirectness (single study, 21 participants, single bedside procedure; Mannucci 1976).

bDowngraded one point due to imprecision (low or absent event incidence, small study).

CAssumed risks taken from available drug product information by Baxter AG, Austria, for the use of Prothromplex TOTAL (HPRA 2018). Prothromplex TOTAL is a 4 factor concentrate (II, VII, IX, and X) with added protein C. Prothromplex, Immuno Vienna as used in Mannucci 1976 was a three-factor concentrate (II, VII, IX) after which factor VII, Immuno, Vienna was administered. No published safety data found for Prothromplex, Immuno, Austria, as used in Mannucci 1976.

$d$ Would have been downgraded two points due to indirectness (differences in plasma preparation in modern practice limit applicability of results from a study conducted in 1976 , and use of prophylactic steroids before plasma transfusion in the study is not recommended by modern guidelines; Mannucci 1976). However downgraded only one point as already downgraded two points in another domain (maximum three downgrade points allowed by GRADE method).

eDowngraded two points due to imprecision (very wide confidence intervals, crossing the line of no difference, which could include both significant harm and benefit, and clinicians instituted a practice change in a single arm during the study, by giving participants prophylactic steroids before FFP transfusion, following four febrile or allergic reactions in the group).

Summary of findings 3. Thromboelastography threshold compared to standard of care (laboratory parameters) for patients undergoing non-cardiac surgery or invasive procedures

Thromboelastography threshold compared to standard of care (laboratory parameters) for patients undergoing non-cardiac surgery or invasive procedures

Patient or population: patients undergoing non-cardiac surgery/invasive procedures

Setting: individuals with cirrhosis undergoing invasive procedures. Study conducted in Italy

Intervention: thromboelastography threshold

Comparison: standard of care (laboratory parameters)

\begin{tabular}{|c|c|c|c|c|c|}
\hline \multirow[t]{2}{*}{ Outcomes } & \multirow{2}{*}{$\begin{array}{l}\text { Relative ef- } \\
\text { fect } \\
(95 \% \mathrm{CI})\end{array}$} & \multicolumn{3}{|c|}{ Anticipated absolute effects ${ }^{\star}(95 \% \mathrm{Cl})$} & \multirow{2}{*}{$\begin{array}{l}\text { Certainty of } \\
\text { the evidence } \\
\text { (GRADE) }\end{array}$} \\
\hline & & $\begin{array}{l}\text { Without } \\
\text { thromboe- } \\
\text { lastography } \\
\text { threshold }\end{array}$ & $\begin{array}{l}\text { With thromboe- } \\
\text { lastography } \\
\text { threshold }\end{array}$ & Difference & \\
\hline All-cause mortality up to $\mathbf{3 0}$ days - not reported & - & - & - & & - \\
\hline $\begin{array}{l}\text { Major bleeding within } 24 \text { hours } \\
\text { № of participants: } 60\end{array}$ & $\begin{array}{l}\text { RR } 0.33 \\
\text { (0.01 to } 7.87 \text { ) }\end{array}$ & \multicolumn{3}{|c|}{ Study population } & $\begin{array}{l}\oplus \ominus \ominus \ominus \\
\text { Very lowa,b,c }\end{array}$ \\
\hline
\end{tabular}




\section{RR 0.33}

(1 RCT)

\section{(0.01 to 7.87$)$}

Study population

\section{Serious adverse events measured by surgery or procedure-related}

complications within $\mathbf{3 0}$ days - not reported

Quality of life - not reported

${ }^{*}$ The risk in the intervention group (and its $95 \%$ confidence interval) is based on the assumed risk in the comparison group and the relative effect of the intervention (and its $95 \% \mathrm{Cl}$ ).

Cl: confidence interval; RCT: randomised controlled trial; RR: risk ratio.

\section{GRADE Working Group grades of evidence.}

High certainty: we are very confident that the true effect lies close to that of the estimate of the effect.

Moderate certainty: we are moderately confident in the effect estimate: the true effect is likely to be close to the estimate of the effect, but there is a possibility that it is

substantially different.

Low certainty: our confidence in the effect estimate is limited: the true effect may be substantially different from the estimate of the effect.

Very low certainty: we have very little confidence in the effect estimate: the true effect is likely to be substantially different from the estimate of effect.

aDowngraded by one point due to high risk of bias across multiple domains (De Pietri 2016).

bowngraded one point due to indirectness (single trial, 60 participants with cirrhosis; De Pietri 2016).

cWould be downgraded two points due to very serious imprecision (low event incidence, very wide confidence intervals including both serious harm and benefit). However downgraded only one point as already downgraded two points in other domains (maximum three downgrade points allowed by GRADE method). 


\section{B A C K G R O U N D}

\section{Description of the condition}

A coagulopathy has been defined as a condition leading to impairment of the blood's clotting ability (Hunt 2014). People undergoing surgical procedures may have a coagulopathy for a myriad of reasons including co-existing medical conditions; nutritional or absorptive abnormalities leading to vitamin $\mathrm{K}$ deficiency (which results in a reduction in vitamin $\mathrm{K}$ dependent clotting factors); abnormal physiological states such as hypothermia or acidosis; coagulant factor dilution due to intravenous fluids or red cell transfusion; use of antiplatelet or anticoagulant medication; or clotting factor consumption due to bleeding (McGilvray 2001).

There are also people requiring surgery in whom both prothrombotic (procoagulant or hyper-coagulant) and coagulopathic (anticoagulant) states may coexist, such as those with liver disease, disseminated intravascular coagulation, renal failure, and systemic inflammatory response syndrome or sepsis (Martlew 2000). People with perioperative critical illness and sepsis are associated with a net procoagulant state, despite laboratory measurements of coagulopathy (McGilvray 2001).

Preoperative screening for people with coagulopathy historically involved measurements of activated partial thromboplastin time (aPTT) and prothrombin time (PT) (van Veen 2011), both of which measure the time for blood to clot, and are affected by the function of different clotting factors. The PT is often presented as the international normalised ratio (INR), which controls for variations in PT measurements due to sensitivity differences among the commercial reagents (Rand 2005).

Clinicians have used abnormal laboratory coagulation results as a marker of coagulopathy, and these abnormalities have formed the rationale for replacing coagulation factors through transfusion of human plasma prophylactically (in the absence of bleeding) before invasive procedures or surgery (Stanworth 2007).

\section{Description of the intervention}

Human plasma is the non-cellular component of blood, containing proteins that help the blood to clot (procoagulants) such as fibrinogen and factors II, V, VII, VIII, IX, X, and XI; anticoagulant proteins $\mathrm{C}$ and $\mathrm{S}$ and antithrombin; and immunoglobulins, water, albumin, and acute phase proteins (Desborough 2015). Plasma is collected either from a single whole blood donation following separation from red cell and platelet components, or from plasmapheresis.

One unit contains a variable volume of plasma (Desborough 2012), typically $200 \mathrm{~mL}$ to $300 \mathrm{~mL}$ (Benjamin 2012), and different preparations are available. Fresh frozen plasma (FFP) is frozen within eight hours of collection and contains greater concentrations of temperature-labile factors $\mathrm{V}$ and VIII than frozen plasma (FP), which is frozen within 24 hours (Benjamin 2012). FFP is stored typically at $-30^{\circ} \mathrm{C}$ for up to 36 months (Norfolk 2013; Stanworth 2007). Once thawed to $1^{\circ} \mathrm{C}$ to $6^{\circ} \mathrm{C}$, FFP retains overall coagulation factor content for up to five days, although factors V and VIII undergo the greatest degradation during this time (Stanworth 2007).
There is also variability of factor concentrations in pathogeninactivated preparations, such as solvent-detergent treated FFP, which contains reduced fibrinogen, factor VIII, and protein S (Norfolk 2013), or methylene blue-treated FFP, which contains reduced fibrinogen and factor VIII (Pamphilon 2000).

Given the variability of clotting factor levels in healthy donors, together with processing, storage, and preparation differences (Stanworth 2007), the potency of coagulation factors in plasma can vary between pooled units from $50 \%$ to $150 \%$ of pooled standardised controls (Benjamin 2012), and even more between units of single donors. Indeed, mean factor VIII concentration has been the only quality-controlled measure for the specification of plasma in the European Union (Stanworth 2007).

\section{Risks associated with the intervention}

Plasma transfusion has the potential to cause life-threatening complications and carries higher risks compared with transfusion of other blood components (Khan 2007; MacLennan 2006). These include transfusion-related acute lung injury (TRALI) (Eder 2007; Holness 2004), transfusion-associated circulatory overload (TACO) (Narick 2012), anaphylaxis or acute allergic reactions - common in $1 \%$ to $3 \%$ of transfusions (Desborough 2015) - ABO incompatibilityinduced haemolysis (Norfolk 2013), multi-organ failure (Watson 2009), and transfusion-transmitted infection. Plasma transfusion is also independently associated with nosocomial infection and sepsis (Karam 2013; Sarani 2008).

Globally, a significant difference exists in the risk of transfusiontransmitted infections between high-income and low-income countries (Dhingra 2013). World Health Organization (WHO) data from 2013 show the incomplete ability of 13 of 173 reporting countries to screen all collected blood for one or more of four transmissible infections - HIV, hepatitis B, hepatitis C, or syphilis - with limited access to test kits representing one such barrier to screening. Furthermore only $66 \%$ of donations in lowincome countries were tested following basic quality-assured procedures (WHO 2017). Blood shortages and an unreliable donor base have historically encouraged the use of paid donors or transfusion without prior testing (WHO 2008). Evidence for increased prevalence and transmission of infections such as HIV in the commercial plasma-donor population has been demonstrated (Volkow 2005; Wu 2001), and this has remained a concern (Abolghasemi 2010). Although an increase of 10.7 million blood donations from voluntary non-renumerated donors between 2008 and 2013 was reported from 159 reporting countries, 71 of 178 countries in 2013 remained dependent on family/replacement and paid donations for more than $50 \%$ of their blood supplies (WHO 2017).

\section{How the intervention might work}

Plasma is a source of procoagulant factors, and a current practice exists to transfuse plasma prophylactically (in the absence of bleeding), based on the rationale that replacing clotting factors through plasma transfusion will correct a coagulopathy and reduce perioperative bleeding risk (Desborough 2012; Rutherford 2008; Stanworth 2007). Reducing this risk has clinical importance given that perioperative bleeding and blood transfusion have been associated with increased morbidity and mortality (Glance 2011; Shander 2007). 
Although further research into exact mechanisms is needed, more recent research has demonstrated that plasma transfusion is associated with protective and restorative effects on the integrity of the lining of blood vessel walls (vascular endothelial glycocalyx layer) (Kozar 2011; Peng 2013; Potter 2015; Rahbar 2015). The lining of blood vessels plays a fundamental role in the initiation and regulation of coagulation, and it is easily damaged by haemorrhagic shock, hypovolaemia, or trauma (Schott 2016).

Prophylactic administration of plasma is often based on mildly deranged laboratory tests (Luk 2002; Palo 2006; Stanworth 2011a; Triulzi 2015), despite evidence that coagulation factors at an INR less than two remain at concentrations adequate to support haemostasis (Deitcher 2002). Furthermore, the degree to which plasma transfusion corrects mildly abnormal coagulation tests is poor (Abdel-Wahab 2006; Holland 2006a; Stanworth 2011a; Williamson 1999).

Second, the underlying premise that abnormal coagulation tests are associated with an increased bleeding risk should be treated with caution (Desborough 2012). Studies suggest no difference in bleeding risk between people with normal or abnormal PT or aPTT undergoing a range of interventions, including spinal surgery (Schramm 2001), angiography (Darcy 1996), liver biopsy (McGill 1990; McVay 1990), thoracocentesis (Puchalski 2013), and abdominocentesis (McVay 1991). These laboratory tests may be prolonged for a variety of reasons (Stanworth 2007), and they are not validated in non-bleeding individuals (Dzik 2004). Their poor predictive value for bleeding risk - as reported in Chee 2008 and Segal 2005 - and their role as a poor marker for haemostasis as discussed in Desborough 2012 and Stanworth 2007 - are not surprising in light of the complexity of haemostatic mechanisms in vivo involving interplay of the endothelium, inflammatory mediators, procoagulant and anticoagulant factors, platelets, and fibrinolysis.

An alternative approach to transfusing plasma based on an INR or PT threshold (which detects only low coagulation factor levels) involves using a test such as rotational thromboelastometry (ROTEM) or thromboelastography (TEG) that assesses how well a blood clot forms in whole blood (haemostasis) (Kinard 2013). ROTEM and TEG not only assess coagulation factor function but also platelet function, strength of the clot, and whether the clot is rapidly broken down (Whiting 2014). TEG may reduce prophylactic transfusions without increasing bleeding complications in people with liver disease undergoing invasive procedures (De Pietri 2016).

These issues may place people with presumed or confirmed coagulopathy undergoing prophylactic FFP transfusion before surgery at risk of potentially life-threatening transfusion-related complications (Khan 2007; MacLennan 2006), without clear evidence that the intervention has benefit.

Furthermore, plasma transfusion is expensive and, together with other blood product use, incurs costs of collecting the product, along with substantial administration costs pertaining to laboratory and staff utilisation, product wastage, and management of transfusion-related complications. These processes generate significant expense for the healthcare provider (Shander 2016; Stokes 2018).

Alternatives to prophylactic plasma transfusion include the following.
- No treatment or placebo.

- Intravenous fluids, including:

* crystalloids, such as saline, dextrose, or balanced electrolyte solutions; or

* colloids, which contain a suspension of macromolecules such as starches, gelatins, or dextrans (Lira 2014).

- Other prohaemostatic agents such as:

* prothrombin complex concentrate, which is produced from plasma and contains a rich source of the vitamin $\mathrm{K}$ dependent factors II, VII, IX, and X in a more concentrated volume compared with plasma;

* cryoprecipitate, which is produced from plasma and is a rich source of fibrinogen, factor VIII, and von Willebrand factor in a concentrated volume and can increase fibrinogen levels with lower transfusion volumes compared with plasma (Norfolk 2013);

* cryosupernatant, which is cryoprecipitate-depleted plasma that is used as an alternative to plasma for individuals with thrombotic thrombocytopenia purpura (O'Shaughnessy 2004);

* fibrinogen concentrate, which contains the substrate converted to fibrin during the final step in the coagulation cascade and formation of a fibrin clot, and may reduce surgical bleeding when administered preoperatively;

* antifibrinolytics, which increase clot strength by inhibiting the body's mechanism for lysis of formed clots; or

* recombinant factor VIIa ( $\mathrm{FFVIIa),} \mathrm{which} \mathrm{is} \mathrm{licensed} \mathrm{for}$ congenital factor VII deficiency, haemophilia, and inhibitory allo-antibodies but is also used off-licence in the setting of uncontrolled haemorrhage refractory to other treatments (Desborough 2016a).

\section{Why it is important to do this review}

There is significant variation in plasma transfusion and prescribing practice (Toumi 2015; Whitaker 2016), together with evidence of inappropriate use (Ejaz 2015; Luk 2002; Moylan 2008; Pahuja 2012; Palo 2006; Prathiba 2001; Stanworth 2011a; Stanworth 2011b; Tinmouth 2013; Triulzi 2015). Furthermore, evidence shows variation in dosing (Stanworth 2011a; Tinmouth 2013; Triulzi 2015), as well as coagulopathy thresholds for transfusion (Stanworth 2011b), with evidence of significant transfusions occurring for mild INR/PT derangement (Ejaz 2015; Triulzi 2015). These variations highlight inconsistencies in management strategies between clinicians.

Although numerous guidelines for plasma usage exist, there is significant variation in the guidance or quality of the evidence base. Some guidelines specifically highlight the absence of high-quality evidence (O'Shaughnessy 2004; Roback 2010; Szczepiorkowski 2013; Yaddanapudi 2014). Another - NICE 2015 recommends plasma transfusion for people undergoing surgery with coagulopathy and risk of significant bleeding based on very low-quality randomised controlled trial (RCT) evidence and the opinion of the Guideline Development Group (Padhi 2015). One guideline recommends that prophylactic transfusion should be avoided (Liumbruno 2009). Another recommends prophylactic transfusion dependent on severity of the coagulation test derangement (Wong 2007), and another recommends seeking specialist advice for consideration of transfusion for people with coagulopathy undergoing intracranial, intraocular, or neuraxial

Prophylactic plasma transfusion for patients without inherited bleeding disorders or anticoagulant use undergoing non-cardiac surgery 
procedures (National Blood Authority 2012). Other guidelines make no mention of prophylactic use in non-cardiac surgery (American Society of Anesthesiologists 2006; American Society of Anesthesiologists 2015).

These variations in FFP usage, dosing, and thresholds, together with variations in guidance, highlight the need to review highquality RCT evidence for the role of plasma. A systematic review first published in 2004 and updated in 2012 demonstrated lack of consistent evidence for prophylactic use across a range of clinical settings (Stanworth 2004; Yang 2012). Although a Cochrane Review has been performed to examine the role of FFP in cardiovascular surgery (Desborough 2015), as yet no Cochrane Review has targeted non-cardiovascular surgery. In the cardiovascular review, 14 trials compared prophylactic use versus no FFP associated with cardiac surgery. Overall, these trials were small and were not powered to determine changes in mortality as a primary outcome. Review authors recommended that large studies are required to assess the therapeutic effects of FFP on clinical outcomes following bleeding.

This Cochrane Review is needed to update previous reviews with recent RCT evidence (Stanworth 2004; Yang 2012), specifically targeting prophylactic use of plasma in non-cardiac surgery or invasive procedures, given that its role is currently uncertain, whereas transfusions carry risk of harm. This review will examine the evidence for FFP compared with no plasma or alternative prohaemostatic agents. It will also examine coagulopathy thresholds for transfusion and will include studies that utilise classical laboratory measurements (PT, INR, aPTT).

\section{OB JECTIVES}

To determine the clinical effectiveness and safety of prophylactic plasma transfusion for people with coagulation test abnormalities (in the absence of inherited bleeding illnesses or use of anticoagulant medication) requiring non-cardiac surgery or invasive procedures.

\section{MET HOD S}

\section{Criteria for considering studies for this review Types of studies}

We included randomised controlled trials (RCTs), with no restriction on language or publication status.

\section{Types of participants}

We included people of all ages with laboratory confirmed or presumed abnormal coagulation (as defined by the study) undergoing non-cardiac surgery or invasive procedures.

We excluded:

- people with clinical evidence of bleeding before enrolment (as they would likely have received blood products); and

- people with inherited bleeding disorders or using anticoagulants (e.g. warfarin, rivaroxaban, apixaban).

We used a broad definition of the term 'surgery' to capture as comprehensive an evidence base as possible. We used the definition created by the American College of Surgeons in 2007 (ACS 2007), given that it encompasses not only the definition of incision and destruction of tissues, but also diagnostic and therapeutic treatments using a variety of instruments including probes or needles (Appendix 1).

We also used the section "Classification of procedures" from the International Classification of Diseases, Ninth Revision, Clinical Modification (ICD9-CM 2011), which classifies operations for guidance. Examples such as oesophago-gastro-duodenoscopy and liver biopsy, or tracheostomy and chest drain insertion, are registered under the group of operative procedures of the digestive system or the respiratory system, respectively.

We excluded studies that assessed only central line insertion because these studies are already included in another Cochrane Review (Hall 2016).

\section{Types of interventions}

We included RCTs comparing three types of plasma transfusion regimens.

- Prophylactic plasma transfusion before surgery/invasive procedures versus no prophylactic plasma transfusion before surgery/invasive procedures (colloid, crystalloid, placebo, or no treatment).

- Plasma transfusion before surgery/invasive procedures compared to alternative pro-haemostatic agents (prothrombin complex concentrate, cryosupernatant, fibrinogen concentrate; antifibrinolytics, and rFVIIa).

- Different haemostatic thresholds for administering a prophylactic plasma transfusion before surgery/invasive procedures (INR, PT, thromboelastography variables).

If sufficient data were available, we would have performed separate meta-analyses for these three comparisons, and we would have assessed age, type and dose of plasma components, and procedure type in subgroup analyses for each of these. We will do this in future updates should the opportunity arise.

\section{Types of outcome measures}

\section{Primary outcomes}

- All-cause mortality (up to 24 hours, and up to 30 days)

- Major bleeding within 24 hours and within seven days as defined by the study, or by the following (based on Schulman 2010).

- Fatal bleeding.

- Intracranial/intraspinal/pericardial/intraocular/ retroperitoneal, into a non-operated joint, or intramuscular causing compartment syndrome.

- Surgical/invasive procedure site bleeding requiring a second intervention or reoperation.

- Surgical/invasive procedure site bleeding that causes a haematoma or haemarthrosis of sufficient size to delay mobilisation or wound healing.

- Surgical/invasive procedure site bleeding that is unexpected and prolonged or causes haemodynamic instability (as defined by the study) and is associated with a $20-\mathrm{g} / \mathrm{L}$ drop in haemoglobin $(\mathrm{Hb})$, or requiring two or more units of whole blood/red cells within 24 hours of bleeding.

- Extrasurgical/invasive procedure site bleeding associated with a $20-\mathrm{g} / \mathrm{L}$ drop in $\mathrm{Hb}$, or requiring two or more units of whole blood/red cells within 48 hours of bleeding.

Prophylactic plasma transfusion for patients without inherited bleeding disorders or anticoagulant use undergoing non-cardiac surgery 


\section{Secondary outcomes}

- Transfusion requirements (within seven days of surgery/invasive procedure).

* Number of individuals requiring a transfusion.

* Mean number of transfusions per participant.

- Use of haemostatic agents (within seven days of surgery/ invasive procedure).

- Volume of blood loss (within seven days of surgery/invasive procedure).

- Serious adverse events (as defined in Appendix 2) due to:

* plasma transfusion (e.g. TRALI, TACO, transfusion-related infection, transfusion-related dyspnoea, acute transfusion reaction) within 24 hours; or

* surgery/invasive procedure (e.g. delayed wound healing, infection) within 30 days after the operation/invasive procedure.

- Resource use: hospital/intensive treatment unit (ITU) length of stay, operating time, return to theatre for management of bleeding.

- Venous and arterial thromboembolism (including deep vein thrombosis; pulmonary embolism; stroke; myocardial infarction) (within 30 days of surgery/invasive procedure, and within 90 days of surgery/invasive procedure).

- Coagulation test abnormalities PT, INR, aPTT, or as defined by the study (within 24 hours of surgery/invasive procedure).

- Quality of life, as defined by individual studies.

\section{Search methods for identification of studies}

The Systematic Review Initiative's Information Specialist working in collaboration with the Cochrane Haematological Malignancies Group devised the search strategy. These are listed in the appendices.

\section{Electronic searches}

We searched the following databases with no limitation on dates or language or publication status. Before review submission, we reran the search and, if additional studies were identified, we would have incorporated these into the review and would have updated findings as required. This strategy served to avoid missing new studies completed during the review process.

Databases searched included the following.

- Cochrane Central Register of Controlled Trials (CENTRAL), in the Cochrane Library (www.cochranelibrary.com/) (Appendix 3).

- MEDLINE (OvidSP, 1946 to 28 January 2019) (Appendix 4).

- Embase (OvidSP, 1974 to 28 January 2019) (Appendix 5).

- Cumulative Index to Nursing and Allied Health Literature (CINAHL) (EBSCOHost) (1937 to 28 January 2019) (Appendix 6).

- PubMed (e-publications and in-process citations ahead of print only) (www.ncbi.nlm.nih.gov/pubmed) (Appendix 7).

- Transfusion Evidence Library (1950 to 28 January 2019) (www.transfusionevidencelibrary.com) (Appendix 8).

- Latin American Caribbean Health Sciences Literature (LILACS) (1982 to 28 January 2019) (lilacs.bvsalud.org/en/) (Appendix 9).

- Web of Science: Conference Proceedings Citation Index-Science (CPCI-S) (Thomson Reuters, 1990 to 28 January 2019) (Appendix $10)$.
Ongoing trial databases included:

- ClinicalTrials.gov (clinicaltrials.gov) (Appendix 11); and

- WHO International Clinical Trials Registry Search Platform (ICTRP) (apps.who.int/trialsearch/) (Appendix 12).

\section{Searching other resources}

We conducted handsearches of the reference lists of included studies and any relevant systematic reviews to identify further relevant studies. We made contact with the lead authors of relevant studies to identify any unpublished material, missing data, or information regarding ongoing studies.

\section{Data collection and analysis}

\section{Selection of studies}

We managed study selection with reference to Chapter 7 of the Cochrane Handbook for Systematic Reviews of Interventions (Higgins 2011a). Two review authors (JH, KW) had planned to independently screen titles and abstracts identified by the search of databases for relevance against the eligibility criteria and immediately excluded clearly irrelevant studies. Given the large numbers of titles and abstracts identified, an additional review author (LE) assisted with the screening process. Two review authors $(\mathrm{JH}, \mathrm{KW})$ screened all titles and abstracts independently. We retrieved full-text papers for all references for which a decision on eligibility could not be made from title and abstract alone.

Three review authors ( $\mathrm{JH}, \mathrm{KW}, \mathrm{LE})$ then assessed the references for relevance from full text. Two (of the three review authors) assessed full text independently. They were not blinded to individual study meta-data such as author, institution, or publication journal. We requested additional information from study authors as necessary to assess the eligibility of individual studies for inclusion.

We used Covidence software to perform simultaneous independent screening and to assist with discrepancy resolution (Covidence 2016). We resolved disagreement between review authors regarding a study's eligibility through discussion and consensus, and through consultation with the third review author as necessary.

We reported the search results and the screening and selection process using a PRISMA flow diagram (Liberati 2009). We recorded the reasons for excluding studies based on full-text assessment and added those to the Characteristics of excluded studies table.

We collated multiple reports of one study so that the study, and not the report, was the unit of analysis.

\section{Data extraction and management}

As recommended in Chapter 7 of the Cochrane Handbook for Systematic Reviews of Interventions (Higgins 2011a), two review authors (JH, LE) independently extracted data using Covidence onto standardised pre-piloted forms, and performed a crosscheck for agreement of data. These review authors were not blinded to names of authors, institutions, journals, or study outcomes. We reported characteristics of the included studies in the Characteristics of included studies table.

We exported data from Covidence into Cochrane's systematic review software Review Manager 5 (RevMan 2014).

Prophylactic plasma transfusion for patients without inherited bleeding disorders or anticoagulant use undergoing non-cardiac surgery 
Data collected included:

- source: study ID, report ID, study author ID, citation and contact details, date of extraction;

- general study details and eligibility: eligibility for inclusion confirmed, reason for exclusion, funding source, conflict of interest declared, references to other relevant studies;

- study methods: location and country, clinical setting, number of centres, study design/type, recruitment dates and study duration, length of follow-up, power calculation, stopping rules, method of sequence generation, method of allocation sequence concealment, method of blinding, bias concerns;

- participant characteristics: age, gender, study population, primary diagnosis and/or operation, baseline laboratory measures of coagulopathy (PT, INR, aPTT, thromboelastography variables, platelet count, $\mathrm{Hb}$ ) or evidence of presumed coagulopathy, total number screened, number included, number excluded, arm sample size, number analysed, number who received treatment, dropout rate, protocol violations, missing data;

- intervention characteristics: number of study arms, description of arms, type of plasma, control product (e.g. crystalloid, colloid, placebo, alternative pro-haemostatic agent), no treatment, haemostatic threshold for administering transfusion, dose of intervention/control; and

- outcomes and results: all-cause mortality within 24 hours and 30 days, major bleeding within 24 hours and seven days, transfusion requirements or number of patients requiring transfusion, use of haemostatic agents, blood loss volume, serious adverse events due to transfusion within 24 hours or surgery within 30 days, operating time, return to theatre for haemostatic control, hospital/critical care length of stay, venous and arterial thromboembolism, change in laboratory measures of coagulation (PT, INR, aPTT, thromboelastography variables) within 24 hours of plasma transfusion, estimate of effects with confidence intervals, key conclusions from study authors, miscellaneous comments from review authors, correspondence with study authors required.

\section{Assessment of risk of bias in included studies}

We assessed the risk of bias for all included RCTs using the Cochrane 'Risk of bias' tool according to Chapter 8 of the Cochrane Handbook for Systematic Reviews of Interventions (Higgins 2011b). Two review authors (JH, LE) worked independently to assess each domain of potential bias listed below as 'high', 'unclear', or 'low' risk of bias. We reported a brief description of the judgement statements upon which we assessed potential bias in the Characteristics of included studies table. We ensured that a consensus on the degree of risk of bias was met through comparison of review authors' statements. If necessary, we had planned to consult with a third review author (KW). We used the Cochrane tool for assessing risk of bias, which included the following domains.

\footnotetext{
- Selection bias.

- Performance bias.

- Detection bias.

- Attrition bias.

- Reporting bias.

- Other bias.
}

\section{Measures of treatment effect}

For continuous outcomes, we recorded mean, standard deviation, and total number of participants in both treatment and control groups. We were unable to perform analyses given the small number of included studies.

In the future, we will perform analyses using the mean difference (MD) with 95\% confidence intervals (Cls) for continuous outcomes using the same scale, and standardised mean difference (SMD) when the scales are different. If available, we will extract and report hazard ratios (HRs) for mortality data. If HRs are not available, we will make every effort to estimate the HR as accurately as possible using available data and a purpose-built method based on the Parmar and Tierney approach (Parmar 1998; Tierney 2007). If sufficient studies provide HRs, we will use HRs in favour of risk ratios (RRs) in a meta-analysis, but for completeness, we will also perform a separate meta-analysis of data from studies providing only RRs for the same outcome.

For dichotomous data, we recorded the number of events and the total number of participants in both treatment and control groups. We were unable to pool data due to the small number of included studies.

In future updates, we will report the pooled RR with $95 \% \mathrm{Cl}$, or when the number of observed events is small ( $<5 \%$ of sample per group) and when trials include balanced treatment groups, we will report the Peto odds ratio (OR) with $95 \% \mathrm{Cl}$ (Deeks 2011). We will report the number needed to treat for an additional beneficial outcome (NNTB) and the number needed to treat for an additional harmful outcome (NNTH), and we will perform quantitative measurements, or will provide a narrative report, as appropriate. If data allow, we will undertake quantitative assessments using Review Manager 5.

\section{Unit of analysis issues}

We did not include any clustered or cross-over trials. In the future, if clustered or cross-over trials will be included, we anticipate that unit of analysis issues may arise with recurring events or multiple treatment events. We would follow guidance from Chapter 16 of the Cochrane Handbook for Systematic Reviews of Interventions (Higgins 2011c). We would report adverse event outcomes as groups of transfusion-related and surgery-related adverse events, as well as venous or arterial events. However often this is not possible due to duplicate counting of the same participant who may have experienced more than one adverse event of the same category (e.g. more than one transfusion-related adverse event). In this case, we would report subgroup categories of adverse events separately and would report the $99 \% \mathrm{Cl}$ of the pooled RR to allow for multiple statistical testing. If this is not possible, we would provide a narrative summary.

\section{Dealing with missing data}

We recorded participants lost to follow-up for each study. We contacted the authors of two trials by email for further information and are currently awaiting responses (Boyd 1996; De Pietri 2016). We utilised the assistance of Cochrane Russia for translation of one study to assess eligibility (Tsermakh 2008).

\section{Assessment of heterogeneity}

Given the small number of included studies, we were unable to combine studies to perform a meta-analysis. In the future, if

Prophylactic plasma transfusion for patients without inherited bleeding disorders or anticoagulant use undergoing non-cardiac surgery 
clinical and methodological characteristics of individual studies are sufficiently homogeneous, we would combine the data to perform a meta-analysis. We would evaluate the extent of heterogeneity by visually inspecting forest plots as well as by utilising statistical methods. We would assess statistical heterogeneity of treatment effects between studies using a $\mathrm{Chi}^{2}$ test (with $\mathrm{P}<0.1$ ). We would quantify heterogeneity using the $\mathrm{I}^{2}$ statistic and would classify it as low $\left(I^{2} \leq 50 \%\right)$, moderate $(50 \%$ to $80 \%)$, or considerable (> 80\%) (Deeks 2011). We would use a randomeffects model for low to moderate statistical heterogeneity given that we anticipate different but related effects across studies. If statistical heterogeneity is considerable, we would not report the overall summary statistic. We would explore potential causes of heterogeneity by performing sensitivity and subgroup analyses as appropriate (Deeks 2011).

\section{Assessment of reporting biases}

We were unable to complete a formal analysis of publication bias, given the small number of included studies. In the future, when at least 10 studies are incorporated into a meta-analysis, we will explore potential publication bias (small-trial bias) by generating a funnel plot and performing an appropriate test for asymmetry as recommended in Chapter 10 of the Cochrane Handbook for Systematic Reviews of Interventions (Sterne 2011).

\section{Data synthesis}

We planned to perform meta-analyses based on recommendations from Chapter 9 of the Cochrane Handbook for Systematic Reviews of Interventions (Deeks 2011). However, we identified an insufficient number of studies to do this.

In the future, if further studies are included, and provided they are sufficiently homogenous in their study design, we will conduct separate meta-analyses (and subgroup analyses) for the three intervention comparisons. In the event of limited quantitative data for statistical analysis and synthesis, we will report findings through qualitative narrative summaries and tables. If sufficient data are available for meta-analysis, we will perform this using Review Manager 5. One review author will enter data into the software programme, which will be independently checked for errors by a second review author. Given the likely variation in intervention practice, we will use a random-effects model in the first instance. We will use the Mantel-Haenszel method for dichotomous data and the inverse variance method for continuous data. We will use the Peto method when event numbers are small.

If heterogeneity is found to be above $80 \%$, and if we identify a cause for the heterogeneity, we will explore this by performing subgroup analyses. If we cannot find a cause for the heterogeneity, we will not perform a meta-analysis but will comment on the results as a narrative while presenting results from all studies in tables.

\section{Subgroup analysis and investigation of heterogeneity}

We planned to carry out separate subgroup analyses. However given the small number of included studies, it was not possible to do this. In the future, if sufficient data are available, we will carry out separate subgroup analyses for the three intervention comparisons to assess heterogeneity for the following.

- Age of individual (neonate, infant, child, adult).

- Type of procedure.
- Plasma type.

- Plasma dose.

We will categorise control interventions into three groups.

- No prophylactic plasma transfusion before surgery (colloid, crystalloid, placebo, or no treatment).

- Alternative pro-haemostatic agents (prothrombin complex concentrate, cryosupernatant, fibrinogen concentrate; antifibrinolytics, and rFVIIa).

- Different haemostatic thresholds for administering a prophylactic plasma transfusion before surgery (INR, PT, thromboelastography variables).

\section{Sensitivity analysis}

We were unable to perform sensitivity analyses due to the small number of included studies. If possible, in the future, we will perform sensitivity analyses to examine the robustness of our findings, by considering only:

- studies with low risk of bias; or

- studies with a low dropout rate $(<20 \%)$.

\section{'Summary of findings' table}

We used GRADEproGDT and the guidance provided in Chapters 11 and 12 of the Cochrane Handbook for Systematic Reviews of Interventions to produce a 'Summary of findings' table for each of the three intervention comparisons (GRADEpro 2015; Schünemann 2011a; Schünemann 2011b). We will utilise the GRADE approach, which defines the quality of the body of evidence as 'high', 'moderate', 'low', or 'very low', based on the following five considerations: design and implementation limitations causing risk of bias, indirectness of evidence, inconsistency or imprecision of results, and risk of publication bias. These tables will include the following outcomes.

- Mortality within 30 days.

- Major bleeding within 24 hours.

- Transfusion requirements measured by mean number of transfusions per participant.

- Transfusion requirements measured by number of individuals requiring a transfusion.

- Serious adverse events measured by plasma transfusion-related complications within 24 hours.

- Serious adverse events measured by surgery-related complications within 30 days.

- Quality of life, as defined by individual studies.

\section{RES U LTS}

\section{Description of studies}

See Characteristics of included studies, Characteristics of excluded studies, Characteristics of studies awaiting classification, and Characteristics of ongoing studies.

\section{Results of the search}

A literature search, conducted by $C D$ and current up to 28 January 2019 , identified a total of 10,448 references plus 758 ongoing studies. After removing duplicates, we screened 6326 references 
and 706 ongoing studies. Any two of three review authors $(\mathrm{JH}$ LE, or KW) excluded 6974 records independently on the basis of the abstract. We retrieved 58 full-text references for independent assessment by the same review authors.
We identified 11 studies within 17 full-text references as potentially eligible for inclusion: five completed studies (De Pietri 2016; Mannucci 1976; Müller 2015; NCT00953901; Veelo 2012); three ongoing studies (NCT02561026; NCT02637427; Smart 2017); and three studies awaiting classification (Boyd 1996; NCT02777424; Tsermakh 2008). (See the PRISMA study flow diagram in Figure 1.) 
Figure 1. PRISMA study flow diagram.

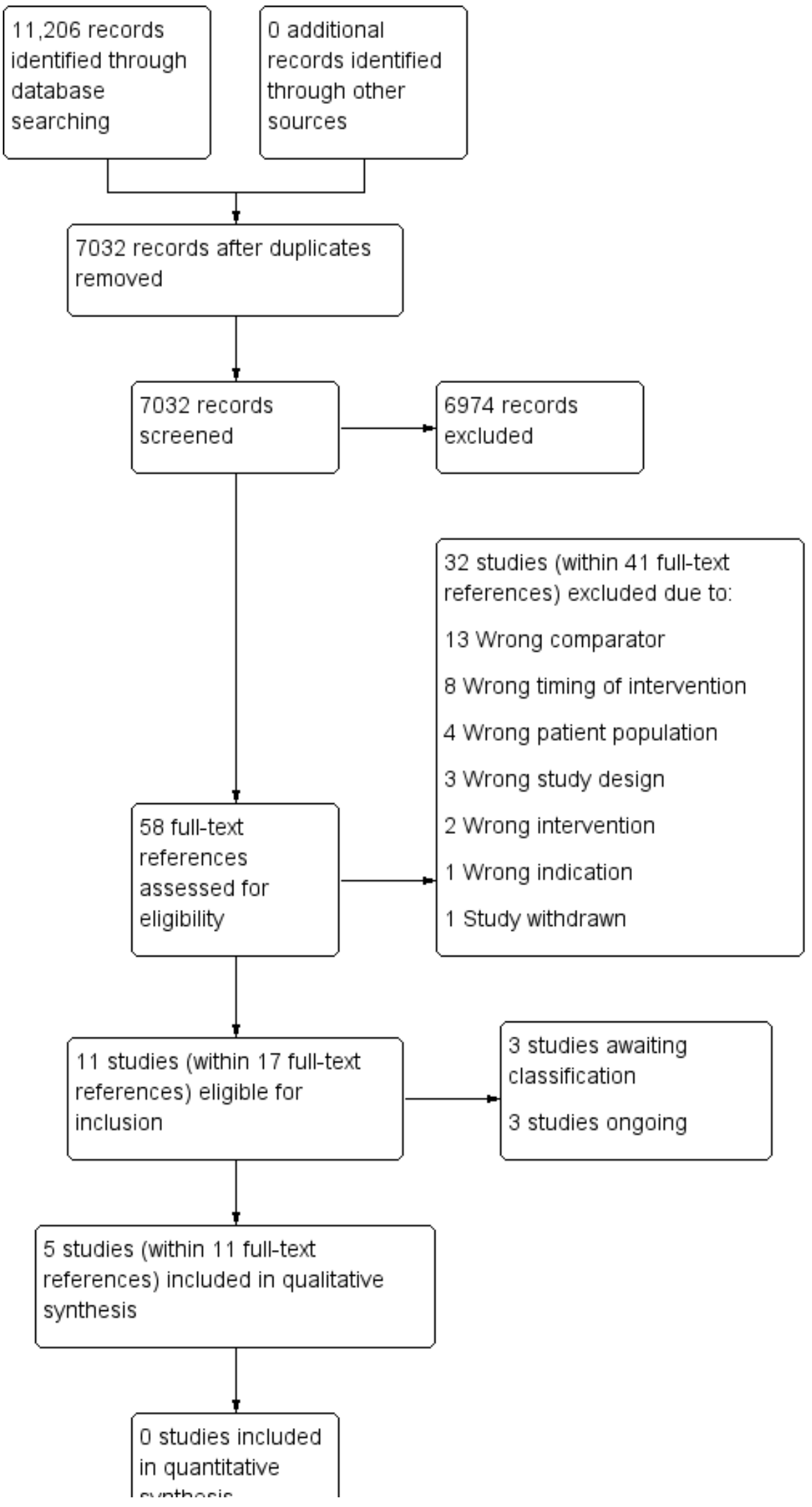

Prophylactic plasma transfusion for patients without inherited bleeding disorders or anticoagulant use undergoing non-cardiac surgery 
Figure 1. (Continued)

\begin{tabular}{|l|} 
in quantitative \\
synthesis \\
(meta-analysis)
\end{tabular}

\section{Included studies}

We included five studies in the review (see Characteristics of included studies).

We grouped the included trials by type of intervention. One trial compared prophylactic plasma transfusion before invasive procedures versus no prophylactic plasma transfusion before invasive procedures (Müller 2015). Another trial compared prophylactic transfusion with FFP or platelets or both with neither FFP nor platelets before invasive procedures (Veelo 2012); one trial compared plasma transfusion before an invasive procedure versus alternative pro-haemostatic agents (prothrombin complex concentrate) (Mannucci 1976); and two trials compared different haemostatic thresholds for administering a prophylactic plasma transfusion before surgical/invasive procedures (INR, PT, thromboelastography variables) (De Pietri 2016; NCT00953901).

\section{Design}

Four trials were published in English between 1976 and 2016. The remaining trial, whose full-text report has not been published, was started in 2006 and was stopped in 2008, after enrolling two participants (NCT00953901). Four studies were parallel-group twoarm studies (De Pietri 2016; Müller 2015; NCT00953901; Veelo 2012). In the fifth trial, after a two-arm study was completed, a third additional arm was added, in which both interventions from the two separate arms were combined (Mannucci 1976).

\section{Sample size}

The trials recruited 236 participants, ranging from 2 in NCT00953901 to 81 in Müller 2015. Three trials were stopped early due to poor recruitment (Müller 2015; NCT00953901; Veelo 2012). The initial plan was to recruit 400 participants in Müller 2015, 188 participants in NCT00953901, and 152 participants in Veelo 2012. Reasons reported for poor recruitment included short time frame for the opportunity to recruit (due to procedure urgency), refusal of consent (Müller 2015), and physician preference for management leading to resistance to recruit (Müller 2015; Veelo 2012).

\section{Setting}

All included studies were set in high-income countries according to the World Bank classification (WB 2017). They were conducted in three countries: two in Italy (De Pietri 2016; Mannucci 1976); two in the Netherlands (Müller 2015; Veelo 2012); and one in the United States (NCT00953901). One was a multi-centre trial incorporating four centres (Müller 2015).

\section{Type of procedure}

Four studies investigated solely bedside invasive procedures (such as chest drain insertion, needle liver biopsy, or abdominal paracentesis) (Mannucci 1976; Müller 2015; NCT00953901; Veelo 2012). One study investigated invasive procedures, which included bedside, radiologically interventional procedures (such as transjugular intrahepatic porto-systemic shunt), other invasive procedures (such as oesophago-gastro-duodenoscopy or colonoscopy), and major surgically operative procedures (17\% of study participants, including hepatic resection, thoracotomy, or other abdominal surgery) (De Pietri 2016). No study solely investigated major operative surgical procedures.

\section{Participants}

Four trials included only adults (De Pietri 2016; Müller 2015; NCT00953901; Veelo 2012), and one study did not report the age of participants (Mannucci 1976). Two trials included only participants with chronic liver disease (De Pietri 2016; Mannucci 1976), and two trials included only participants in intensive care (Müller 2015; Veelo 2012).

One study excluded participants taking anticoagulants or antiplatelets at the time of, or within seven days of, enrolment (De Pietri 2016). One study excluded participants with a bleeding time greater than seven minutes (Mannucci 1976). Another study excluded participants with an INR greater than three, and those taking vitamin $\mathrm{K}$ antagonists, activated protein $\mathrm{C}$, abciximab, tirofiban, ticlopidine, or prothrombin complex concentrates, although heparin and low molecular weight heparin were not exclusion criteria provided they were discontinued for an 'appropriate period' (Müller 2015). One study excluded participants with prothrombin time greater than 20 seconds and those receiving clopidogrel (Veelo 2012).

\section{Interventions}

One study compared solely the use of FFP versus no FFP (Müller 2015). Another study compared the use of FFP or platelets or both with neither FFP nor platelets (Veelo 2012). One study compared use of FFP versus use of factors II, IX, and X followed by VII (Mannucci 1976). Two studies compared use of FFP versus different coagulopathy test thresholds (De Pietri 2016; NCT00953901).

\section{Studies comparing FFP vs no FFP}

One study compared FFP transfusion versus no FFP transfusion (Müller 2015). The other study compared transfusion with FFP or platelets (if low count and/or acetylsalicylic acid used) or both with neither FFP nor platelet transfusion (Veelo 2012). In this study, of the 35 participants randomised to the correction arm, $18(51 \%)$ underwent FFP transfusion, and $23(66 \%)$ underwent platelet transfusion. Given that more than $50 \%$ of participants in the group received FFP, and given the paucity of trials available for inclusion, we currently included this study and its data in the review. However, we also contacted the first author to request data related only to participants who received FFP. We are grateful for her acknowledgement of this request, and we are awaiting further correspondence.

\section{Studies comparing FFP vs alternative pro-haemostatic agents}

One study compared FFP versus factors II, IX, and X, followed by VII (Mannucci 1976). In this study, after completing recruitment

Prophylactic plasma transfusion for patients without inherited bleeding disorders or anticoagulant use undergoing non-cardiac surgery 
of participants to the two arms, researchers recruited a further cohort of participants to each receive a combination of FFP and prothrombin complex concentrate (PCC), thereby subsequently generating a third (non-parallel) arm.

\section{Studies comparing different transfusion thresholds}

One study compared transfusing FFP using a thromboelastography R-time threshold $>40$ minutes as a transfusion trigger versus using an INR $>1.8$ as a transfusion trigger (De Pietri 2016). Another study compared liberal (to keep INR < 1.6) versus restrictive (INR 1.6 to 3 ) FFP transfusion regimens (NCT00953901).

\section{Outcomes}

No studies measured all primary outcomes defined by the review. Two studies measured all-cause mortality up to 24 hours - Müller 2015 - or at 30 days - Veelo 2012 - as defined in the review criteria above. One study measured 90-day mortality (De Pietri 2016). We contacted study authors for information regarding 24-hour and 30day mortality, and we are currently awaiting a response.

Four studies measured major bleeding within 24 hours (De Pietri 2016; Mannucci 1976; Müller 2015; Veelo 2012), and one study within seven days (De Pietri 2016).

No studies measured all secondary outcomes defined by the review.

\section{Ongoing studies}

We identified three ongoing studies (NCT02561026; NCT02637427; Smart 2017). Both NCT02561026 and NCT02637427 are pilot studies determining feasibility for a large trial (Characteristics of ongoing studies). NCT02561026 is an open-label parallel-group two-arm trial conducted in Canada to compare prophylactic FFP transfusion versus no transfusion before an invasive procedure. This multicentre trial aimed to recruit 80 participants across three hospitals in an intensive care unit setting. Recruitment was expected to be complete by August 2018.

NCT02637427 is a parallel-group two-arm, single-blinded (to outcome assessor) trial conducted in the United States to compare prophylactic FFP transfusion versus no transfusion before an invasive procedure outside of the operating room. This multi-centre trial aimed to recruit 110 participants over two locations. It is expected to complete recruitment by April 2020.

Smart 2017 is a parallel-group, two-arm, prospective, randomised controlled trial comparing blood product use, bleeding events, and costs during and after endoscopic procedures in participants with liver cirrhosis with transfusion as guided by thromboelastometry versus conventional coagulation tests. An abstract has been published, and a full study report has not been published.

\section{Studies awaiting classification}

Three studies are awaiting classification (Boyd 1996; NCT02777424; Tseimakh 2008). The timing of the intervention in Boyd 1996 requires further clarification. Following contact with the study author, we are awaiting further information. In NCT02777424, the need for 'surgery' is not an absolute requirement to meet the study's inclusion criteria for enrolment. Given that the review's inclusion criteria require that participants undergo either surgery or invasive procedures, further assessment of this study will be needed following its completion to determine whether a subgroup of participants undergoing surgery (or invasive procedures) can be included in the review. Tseimakh 2008 was written in Russian, and we are awaiting a translation of the full text by Cochrane Russia. See Characteristics of studies awaiting classification for further information.

\section{Excluded studies}

We excluded 32 studies within 41 full-text references after assessing their eligibility through a full-text review (see Characteristics of excluded studies).

We excluded:

- 13 studies as the comparator did not meet our eligibility criteria (ChiCTR-INR-17013901; Freeman 1998; Galganski 2017; Laine 2003; Lerner 1997; Mintz 2006; NCT00235183; NCT03700723; Palmieri 2013; Ramies 2002; Tinmouth 2008; Wieding 1999; Williamson 1999);

- eight studies as timing of the intervention did not meet our eligibility criteria (Hedstrand 1987; Liu 1994; NCT02352181; NCT00994045; Pieters 2015; Sommoggy 1990; Urwyler 2009; Wang 2010);

- four studies as the participant population did not meet our eligibility criteria (Cao 2016; Jilma-Stohlawetz 2011; Lance 2012; NCT00302965);

- three studies as the study design did not meet our eligibility criteria (Gazzard 1975; Hildebrandt 2007; Kerner 2008);

- two studies as the intervention did not meet our eligibility criteria (Bauer 1986; NCT00656396);

- one study as the indication for the intervention did not meet our eligibility criteria (Rocha 2017); and

- one study as the study was withdrawn before participant enrolment was complete (NCT00233246).

\section{Risk of bias in included studies}

See Figure 2 and Figure 3 for visual representations of 'Risk of bias' assessments across all included trials and for each individual item in the included trials. See the Characteristics of included studies section 'Risk of bias' table for further information about bias identified within individual trials. 
Figure 2. Risk of bias summary: review authors' judgements about each risk of bias item for each included study.

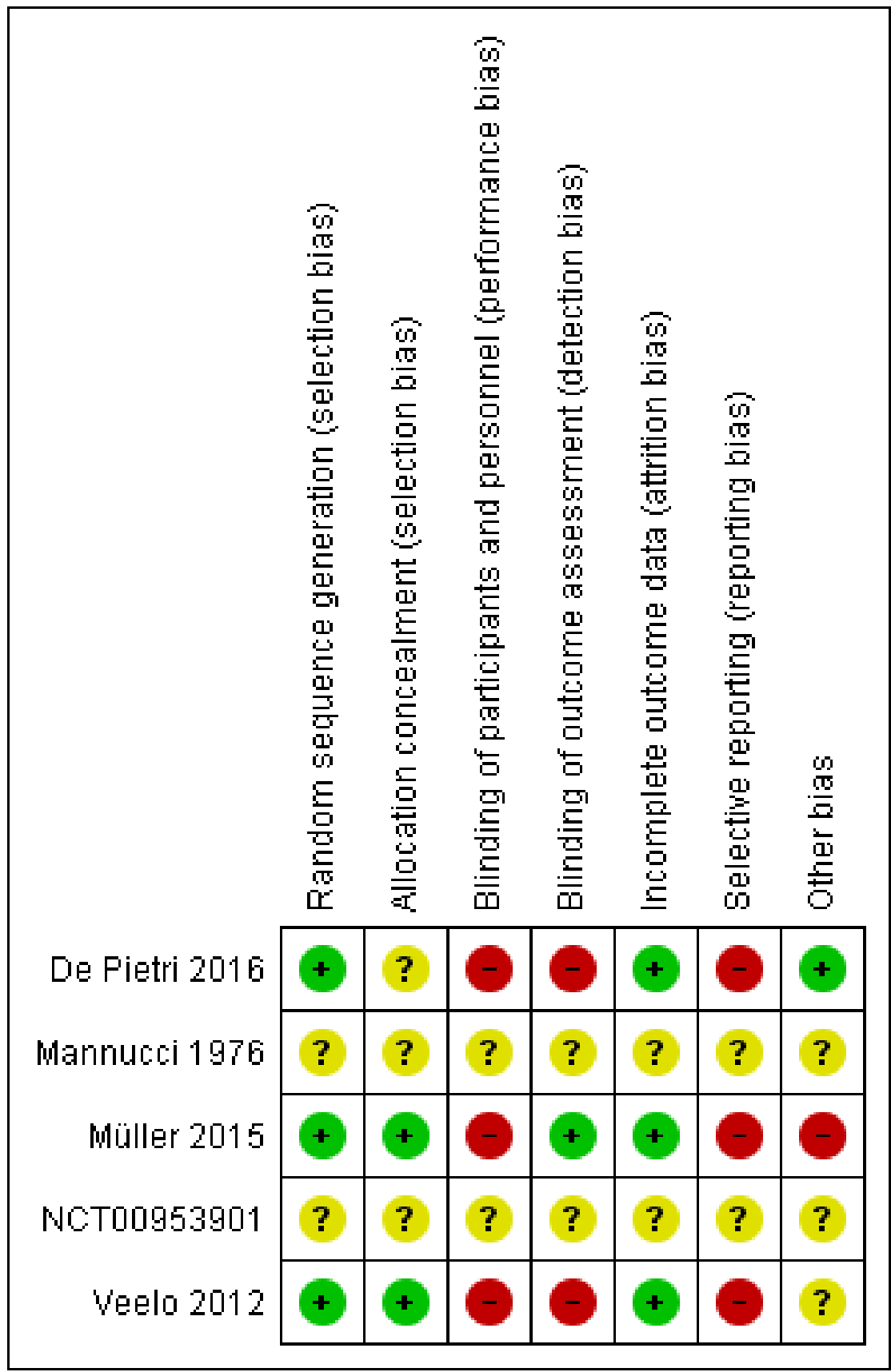


Figure 3. Risk of bias graph: review authors' judgements about each risk of bias item presented as percentages across all included studies.

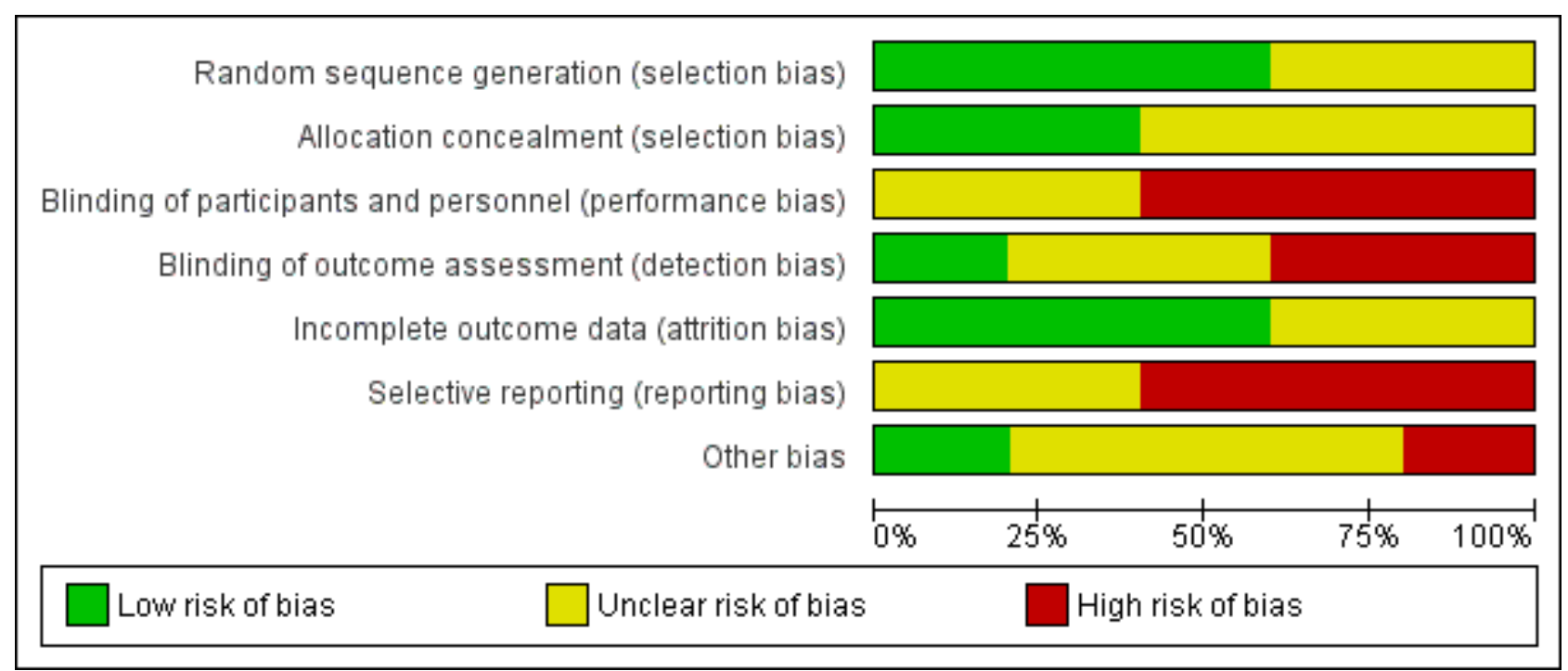

One study was completed in 2008 after recruiting two patients (NCT00953901). No report has been published, thereby precluding an analysis.

\section{Allocation}

Two trials were at low risk of selection bias due to adequate methods of sequence generation and allocation concealment (Müller 2015; Veelo 2012).

\section{Random sequence generation}

Three trials were at low risk of bias due to random sequence generation because they used computer-generated - Müller 2015 and Veelo 2012 - or electronically generated randomisation (De Pietri 2016).

One trial was at unclear risk of bias because it did not provide any

\section{Allocation concealment}

Two trials were at low risk of bias due to allocation concealment because they used either a web-based permuted block system - Müller 2015 - or consecutively numbered, opaque, sealed envelopes (Veelo 2012).

Two trials were at unclear risk of bias due to allocation concealment. Mannucci 1976 used a system of sealed envelopes, but it is unclear from the report whether opaque, sequentially numbered, sealed envelopes were used. De Pietri 2016 provided no information about the method of allocation concealment.

\section{Blinding}

\section{Blinding of participants and personnel}

One study was at unclear risk of performance bias because information in the study report was insufficient to make an assessment (Mannucci 1976). details about the method of sequence generation (Mannucci 1976).

Three studies were at high risk of performance bias (except for allcause mortality) because of their open-label design (De Pietri 2016; Müller 2015; Veelo 2012).

\section{Blinding of outcome assessors}

One study was at low risk of detection bias, given its blinded endpoint evaluation design, which blinded the outcome assessor from the intervention during assessment of bleeding (Müller 2015).

One study was at unclear risk of detection bias given absence of information in the study report to permit assessment (Mannucci 1976).

Two studies were at high risk of bias (except for all-cause mortality) because of their open-label design (De Pietri 2016; Veelo 2012).

\section{Incomplete outcome data}

Three studies were at a low risk of attrition bias. In one study, all randomised participants were included in an intention-to-treat (ITT) analysis (De Pietri 2016). In another study, all participants who were randomised and underwent a procedure were included (Müller 2015). In the third study, a clear CONSORT diagram was reported, demonstrating an equal number of participants in each study arm who did not undergo the procedure after randomisation (Veelo 2012).

One study was at an unclear risk of attrition bias because information was insufficient to permit assessment (Mannucci 1976).

\section{Selective reporting}

One study was at unclear risk of bias as no protocol or trial registration was found, thereby precluding assessment (Mannucci 1976).

Three studies were at high risk of bias (De Pietri 2016; Müller 2015; Veelo 2012). One study recognised limitations in an outcome assessment method, leading to risk of under-reporting (Müller 2015). In another study, the time span for the primary outcome was

Prophylactic plasma transfusion for patients without inherited bleeding disorders or anticoagulant use undergoing non-cardiac surgery 20 or invasive procedures (Review)

Copyright $\odot 2019$ The Cochrane Collaboration. Published by John Wiley \& Sons, Ltd. 
not clearly reported (De Pietri 2016), whereas in yet another study, the primary outcomes were not defined in the report (Veelo 2012). Two studies reported an outcome that was not predefined in their protocol - De Pietri 2016 - or public registry (Veelo 2012). All three studies did not report an outcome that had been predefined in their protocol - De Pietri 2016 and Müller 2015 - or in the public registry (Veelo 2012).

\section{Other potential sources of bias}

\section{Balance of baseline characteristics}

One study was at low risk of bias because it reported and demonstrated similar baseline characteristics between arms (De Pietri 2016).

Two studies were at unclear risk of bias. The first study did not report participant baseline characteristics (Mannucci 1976). The second reported baseline characteristics but did not highlight nor discuss a statistically significant difference (among patients with expected prolonged duration of mechanical ventilation) between arms (Veelo 2012). The material risk of this difference is unclear.

One study was at high risk of bias because it reported a statistically significant imbalance in participants with liver disease between groups (Müller 2015).

\section{Early termination of study}

One study was at unclear risk of bias because it was terminated early after randomising only $47 \%$ of its recruitment target due to increasing clinician resistance to transfusing FFP following recognition of a low incidence of bleeding in the no FFP group (Veelo 2012).

One study was at high risk of bias because it was terminated early after randomising only $20 \%$ of its recruitment target owing to a slow rate of inclusion (Müller 2015). As a result, study authors could not demonstrate non-inferiority between the two arms.

\section{Effects of interventions}

See: Summary of findings for the main comparison Prophylactic plasma transfusion before surgery/invasive procedures compared to no prophylactic plasma transfusion before surgery/invasive procedures (colloid, crystalloid, placebo, or no treatment) for patients undergoing non-cardiac surgery or invasive procedures; Summary of findings 2 Prophylactic plasma transfusion before surgery/invasive procedures compared to alternative haemostatic agents for patients undergoing non-cardiac surgery/invasive procedures; Summary of findings 3 Thromboelastography threshold compared to standard of care (laboratory parameters) for patients undergoing non-cardiac surgery or invasive procedures

See Summary of findings for the main comparison, Summary of findings 2, and Summary of findings 3 for the main comparisons.

\section{Studies comparing fresh frozen plasma (FFP) vs no FFP}

One study compared FFP transfusion versus no FFP transfusion (Müller 2015). Another study compared FFP or platelet transfusion or both versus neither FFP nor platelet transfusion (Veelo 2012).

\section{Primary outcomes \\ Mortality up to 24 hours and up to 30 days}

One study reported all-cause mortality up to 24 hours (Müller 2015). We are uncertain whether there is any difference between the two groups ( 1 trial, 76 participants; risk ratio (RR) $0.70,95 \%$ confidence interval (Cl) 0.48 to 1.03 ; low-quality evidence) (Analysis 1.1).

Another study reported intensive care unit (ICU) mortality up to 30 days (Veelo 2012). We are very uncertain whether there is any difference between the two groups ( 1 trial, 72 participants; RR 0.38 , $95 \% \mathrm{Cl} 0.13$ to 1.10 ; very low-quality evidence) (Analysis 1.1).

\section{Major bleeding within $\mathbf{2 4}$ hours and within seven days}

Two studies reported major bleeding at 24 hours (Müller 2015; Veelo 2012).

In the study comparing FFP transfusion versus no transfusion (Müller 2015), we are very uncertain whether there is any difference in major bleeding between the two arms ( 1 trial, 76 participants; RR $0.33,95 \% \mathrm{Cl} 0.01$ to 7.93 ; very low-quality evidence) (Analysis 1.2). A single bleeding event (haemothorax) was reported following the invasive procedure (chest drain insertion) in a participant assigned to the non-transfusion group, which fulfilled the above criteria of major bleeding (requiring 3 units of red blood cells (RBCs), 3 units of FFP, and 1 unit of platelets). Haemostasis was achieved following this treatment.

In the study comparing FFP or platelet transfusion or both versus neither FFP nor platelet transfusion, we are very uncertain whether there is any difference in major bleeding between the two groups (1 trial, 72 participants; RR $1.59,95 \% \mathrm{Cl} 0.28$ to 8.93 ; very low-quality evidence) (Analysis 1.2) (Veelo 2012).

No study reported bleeding within seven days.

\section{Secondary outcomes}

\section{Transfusion requirements within seven days}

One study reported the number of participants requiring transfusion (Veelo 2012), although the time scale for this outcome was not reported. No RBC transfusion occurred in either group (1 trial, 72 participants; very low-quality evidence).

One study reported transfusion requirements as median number (and interquartile range (IQR)) of transfusions per participant in the first 24 hours only (Müller 2015; Table 1). Study authors reported no difference in RBCs, FFP, or platelet transfusion between groups (1 trial, 76 participants; RBC $P=0.91$, FFP $P=0.06$, platelets (PLTs) $P=$ 0.43 ; very low-quality evidence).

\section{Use of haemostatic agents within seven days}

No study reported this outcome.

\section{Volume of blood loss within seven days}

One study reported this outcome as median (grams) blood loss (with IQR) per participant, although the duration of assessment for this outcome is unclear from the report (Veelo 2012). Study authors reported no difference between the two study arms (1 trial, 72 participants; median 3.0 grams, IQR 1.0 to 6.0 in the intervention group vs median 3.0 grams, IQR 2.0 to 6.0 in the no intervention group $(P=0.96$; Table 1$)$. 
Serious adverse events from plasma transfusion (within 24 hours) or from surgery/intervention (within 30 days)

One study reported a lung injury score at 24 hours after randomisation as median and IQR (Müller 2015). Study authors reported that a difference between the two groups "did not reach statistical significance" (1 trial, 76 participants; median 2, IQR 0.8 to 2.5 in the intervention arm vs median 1.25 , IQR 0.4 to 2.4 in the no intervention $\operatorname{arm}(P=0.28$; Table 1$)$. Study authors did not provide quantitative data regarding baseline lung injury scores for the two arms nor changes in score between baseline and 24 hours post procedure. They qualitatively describe in the report's narrative that baseline scores were increased in both groups.

No study reported surgical or procedure-related adverse events (excluding bleeding).

\section{Resource use}

\section{Hospital length of stay}

No study reported this outcome.

\section{ICU length of stay}

Two studies reported ICU length of stay as median and IQR (Table 1) (Müller 2015; Veelo 2012). Study authors from both studies showed no evidence of a difference between the two groups (Müller 2015: 76 participants, $\mathrm{P}=0.13$; Veelo 2012: 72 participants, $\mathrm{P}=0.21$ ).

\section{Duration of operation/intervention}

No study reported this outcome.

\section{Return to theatre/intervention room}

One study reported a single major bleeding event within 24 hours (Müller 2015). However study authors did not report the need to transfer/return to the theatre/intervention room for intervention to achieve haemostasis, although the follow-up period was not described in the study report. Therefore no events were reported for this outcome (1 trial, 76 participants).

\section{Venous and arterial thromboembolism within $\mathbf{3 0}$ days and 90 days}

No study reported this outcome.

\section{Coagulation test abnormalities}

One study reported this outcome as median international normalised ratio (INR) and IQR before and after transfusion (Table 1) (Müller 2015). Study authors reported a reduction in INR following FFP transfusion (1 trial, 38 participants; median 1.8, IQR 1.5 to 2.5 before FFP transfusion vs median 1.4, IQR 1.3 to 1.63 after FFP transfusion; $\mathrm{P}<0.001)$. However only 21 of 38 participants $(54 \%)$ demonstrated a reduction in INR $<1.5$ after transfusion.

\section{Quality of life}

No study reported this outcome.

\section{Studies comparing FFP vs other pro-haemostatic alternatives}

Only one study compared FFP versus factors II, IX, X, and VII (Mannucci 1976).

\section{Primary outcomes}

\section{Mortality up to 24 hours and up to 30 days}

\section{Major bleeding within $\mathbf{2 4}$ hours and within seven days}

Mannucci 1976 reported this outcome, although the time span for its measurement is unclear from the report. The study followed up with participants for 12 months. No major bleeding events were reported in either group ( 1 trial, 21 participants; very low-quality evidence). We are very uncertain of the significance of this result, given the very low quality of evidence for this outcome.

\section{Secondary outcomes}

Transfusion requirements within seven days

Mannucci 1976 did not report this outcome.

Use of haemostatic agents within seven days

Mannucci 1976 did not report this outcome.

Volume of blood loss within seven days

Mannucci 1976 did not report this outcome.

Serious adverse events from plasma transfusion (within $\mathbf{2 4}$ hours) or from surgery/intervention (within 30 days)

Mannucci 1976 reported serious adverse events from plasma transfusion (defined by the criteria in Appendix 2). We are very uncertain whether there was any difference in these events between the two groups ( 1 trial, 21 participants; RR 9.82, 95\% Cl 0.59 to 162.24 ; very low-quality evidence) (Analysis 2.1). During the study period, following four events in the FFP group, there was a practice change whereby all subsequent participants in the FFP group received prophylactic steroids, which may have reduced the rate of, or clinical detection of, future events, and may have masked their true incidence. The study was also likely underpowered to detect a difference between groups.

Mannucci 1976 did not report serious adverse events related to the procedure within 30 days.

\section{Resource use}

Mannucci 1976 did not report hospital length of stay, ICU length of stay, procedure duration, or need to return to the theatre/ procedure room.

\section{Venous and arterial thromboembolism within 30 days and 90 days}

We are very uncertain whether there is a difference in thromboembolism with the use of FFP. Mannucci 1976 reported no cases of thromboembolism in the groups, although the timing of assessment of this outcome was not reported ( 1 trial, 21 participants; very low-quality evidence). Participants were followed up for 12 months.

\section{Coagulation test abnormalities}

Mannucci 1976 reported the number of participants with corrected indices of coagulation. We are very uncertain whether there is a difference between the groups of participants with persistently deranged indices ( 1 trial, 21 participants; aPTT: RR $2.75,95 \% \mathrm{Cl} 0.68$ to 11.13 ; PT: RR $0.44,95 \%$ Cl 0.2 to 0.96 ; Normotest: RR $0.43,95 \% \mathrm{Cl}$ 0.21 to 0.88 ; very low-quality evidence) (Analysis 2.2).

\section{Quality of life}

Mannucci 1976 did not report this outcome.

Mannucci 1976 did not report this outcome.

Prophylactic plasma transfusion for patients without inherited bleeding disorders or anticoagulant use undergoing non-cardiac surgery 


\section{Studies comparing different transfusion thresholds}

Two studies compared different transfusion thresholds. One study compared a liberal (to keep INR < 1.6) versus restrictive (INR 1.6 to 3) FFP transfusion regimen (NCT00953901). However this study ceased recruitment after enrolling two participants and reported no outcomes.

Another study compared FFP transfusion using a thromboelastography R-time threshold $>40$ minutes as a transfusion trigger vs a transfusion trigger with INR $>1.8$ (De Pietri 2016).

\section{Primary outcomes}

\section{Mortality up to 24 hours and up to $\mathbf{3 0}$ days}

No study reported 24-hour or 30-day mortality. One study reported all-cause mortality up to 90 days (De Pietri 2016). We are very uncertain whether there is a difference between groups ( 1 trial, 60 participants; RR $1.14,95 \% \mathrm{Cl} 0.47$ to 2.75 ; very low-quality evidence) (Analysis 3.1).

\section{Major bleeding within 24 hours and within seven days}

One study reported data for this outcome (De Pietri 2016). One participant $(1 / 30)$ developed major bleeding according to the above criteria on the day following the invasive procedure (paracentesis), requiring packed red cells and FFP transfusions. This participant had been randomised to the standard of care group and had received FFP before undergoing the procedure. There were no events in the thromboelastography (TEG) group $(0 / 30)$. We are very uncertain whether there is a difference between the groups (1 trial, 60 participants; RR $0.33,95 \% \mathrm{Cl} 0.01$ to 7.87 ; very low-quality evidence) (Analysis 3.2).

\section{Secondary outcomes}

\section{Transfusion requirements within seven days}

One study reported data for this outcome (De Pietri 2016). The number of individuals requiring overall blood products in the TEG group may be reduced versus the number in the standard of care (SOC) group (1 trial, 60 participants; RR $0.18,95 \% \mathrm{Cl} 0.08$ to 0.39 ; low-quality evidence). However we are very uncertain whether there was a reduction in participants within the TEG group requiring either FFP alone ( 1 trial, 60 participants; RR $0.03,95 \% \mathrm{Cl} 0.00$ to 0.48 ; very low-quality evidence), PLTs alone (1 trial, 60 participants; RR $0.20,95 \% \mathrm{Cl} 0.05$ to 0.84 ; very low-quality evidence), or both FFP and PLTs ( 1 trial, 60 participants; RR $0.75,95 \% \mathrm{Cl} 0.18$ to 3.07 ; very low-quality evidence) (Analysis 3.3).

No study reported the mean number of transfusions per patient.

\section{Use of haemostatic agents within seven days}

No study reported this outcome.

\section{Volume of blood loss within seven days}

No study reported this outcome.

Serious adverse events from plasma transfusion (within $\mathbf{2 4}$ hours) or from surgery/intervention (within 30 days)

One study reported an allergic reaction during FFP transfusion in the SOC group (1/30) compared to none in the TEG group (0/30) (De Pietri 2016). We are very uncertain whether there was a difference between groups ( 1 trial, 60 participants; RR $0.33,95 \% \mathrm{Cl} 0.01$ to 7.87 ; very low-quality evidence) (Analysis 3.4).

No study reported surgical or procedure-related serious adverse events (excluding bleeding, as already described above and in Analysis 3.2).

\section{Resource use}

No study reported hospital length of stay, ICU length of stay, or procedure duration. Studies did not describe the necessity to return to the theatre or the intervention room to control bleeding in the participant with a post-procedural bleeding event.

Venous and arterial thromboembolism within 30 days and within 90 days

No study reported this outcome.

Coagulation test abnormalities

No study reported this outcome.

\section{Quality of life}

No study reported this outcome.

\section{DISCUSSION}

The objective of this review was to determine the clinical effectiveness and safety of prophylactic plasma transfusion for people with confirmed or presumed coagulopathy requiring noncardiac surgery or invasive procedures.

Five trials were eligible for inclusion in this systematic review (De Pietri 2016; Mannucci 1976; Müller 2015; NCT00953901; Veelo 2012). One study was terminated after recruiting only two patients; this study report has not been published (NCT00953901). No results can be ascertained from this study.

Of the four completed studies, all were carried out in high-income countries between the years 1976 and 2016; they involved a total of 234 participants, all of whom were $>18$ years of age.

Two trials studied participants in an intensive care unit (ICU) setting (153 participants) (Müller 2015; Veelo 2012). Two trials studied only procedures carried out by participants' bedside (153 participants; in Müller 2015 and Veelo 2012, although seven participants in Veelo 2012 subsequently underwent surgical tracheotomy as opposed to percutaneous dilatational tracheotomy due to landmark recognition difficulties). Only one trial studied participants who underwent surgical procedures in an operating theatre such as hepatic resection, other abdominal surgery, or thoracotomy (10 participants out of a total of 60) (De Pietri 2016).

Only one trial studied fresh frozen plasma (FFP) as a single intervention compared with no intervention (Müller 2015). Another study compared FFP or platelet transfusion or both versus neither FFP nor platelet transfusion (Veelo 2012). A single trial studied the use of FFP versus the use of a combination of alternative pro-haemostatic agents - Prothromplex (containing factors II, IX, and X), followed by factor VII concentrate (Mannucci 1976). A single study compared different haemostatic thresholds for FFP transfusion, reporting a thromboelastography (TEG)-measured Rtime $>40$ minutes compared with an international normalised ratio (INR) >1.8 (De Pietri 2016). 


\section{Summary of main results}

Given the very low-quality evidence, we are very uncertain whether there is a difference in:

- mortality with the use of prophylactic FFP versus no FFP. No trials studying other eligible comparators reported mortality up to 30 days;

- major bleeding within 24 hours, whether compared with no FFP or alternative pro-haemostatic agents, or using different transfusion thresholds. Few or no events in the studies and small study sample sizes have contributed to this uncertainty;

- transfusion requirements, in the form of number of transfusions per participant (one study comparing FFP transfusion vs no transfusion) or number of participants requiring a transfusion (one study comparing FFP or platelet transfusion or both vs neither FFP nor platelet transfusion);

- serious adverse events from FFP transfusion versus no transfusion or other pro-haemostatic agents, or using different thresholds for transfusion;

- surgical/interventional adverse events in participants transfused with FFP using a TEG-guided transfusion trigger versus standard tests for coagulation. No trials comparing FFP use versus no FFP use or use of an alternative pro-haemostatic product reported this outcome; or

- arterial or thromboembolism rate. Only one study, which compared participants receiving FFP versus factors II, IX, X, and VII, reported this outcome (Mannucci 1976).

For participants receiving FFP transfusion via a TEG-guided transfusion trigger versus standard tests of coagulation (INR), the number of participants subsequently requiring transfusion (for overall blood products) via a TEG-guided trigger may have been reduced. Our confidence is limited due to the low quality of evidence for this outcome. However we are very uncertain whether there is a difference in individual product use (FFP, platelets, or both), given that the evidence is of very low quality.

No studies reported the outcome looking at quality of life.

\section{Overall completeness and applicability of evidence}

This review aimed to provide the most up-to-date and comprehensive assessment of the clinical effectiveness and safety of prophylactic plasma transfusion for people with confirmed or presumed coagulopathy requiring non-cardiac surgical or invasive procedures.

To facilitate development of wide and comprehensive criteria for inclusion, we used a broad definition of surgical or surgically interventional procedures. We used the definition of the American College of Surgeons, which includes consideration of the use of probes or needles. We also used the classification system from the International Classification of Diseases, Ninth Revision, Clinical Modification (ICD9-CM 2011), which codifies various surgically oriented or interventional procedures within its operative classification.

Our search in any language has resulted in consideration of a foreign language trial, resulting in collaboration with Cochrane Russia for its translation (Tseĭmakh 2008).
We sought contact with the author of one study for further information required to determine its eligibility for inclusion based on our criteria (Boyd 1996). A second study awaiting classification is still ongoing (NCT02777424). Both studies compare use of FFP versus an alternative pro-haemostatic agent. Two ongoing studies are comparing FFP use versus no transfusion (NCT02561026; NCT02637427), and one ongoing study is comparing transfusion of blood products guided by thromboelastometry versus conventional coagulation tests (Smart 2017).

Several limitations may affect the strength and applicability of our review.

- Two studies (153 participants) of the four included in this review (234 participants) were set in the intensive care unit (Müller 2015; Veelo 2012). Given that these studies represent $65 \%$ of participants included in this review on which review findings are based, and given that individuals in the ICU represent more severe acute illness or physiological derangement compared with other individuals with a coagulopathy requiring surgery or interventional procedures, caution is required in extrapolating or generalising these findings to other person cohorts.

- Of the four included studies, two trials studied bedside interventional procedures (Müller 2015; Veelo 2012), and one trial studied percutaneous needle liver biopsy (likely carried out in an interventional area or theatre, although the locality is not clear from the report) (Mannucci 1976). Only a single trial included major operative surgery such as solid organ (liver) resection, other abdominal surgery, or thoracotomy (together with other invasive procedures) (De Pietri 2016). Major general surgery was therefore under-represented by the included studies, and no included studies represented major head and neck, neurological, or orthopaedic surgery.

- No trials eligible for inclusion in the review studied children.

- Significant variability is evident in methods used by the four studies for assessing, grading, and reporting bleeding. One study did not report its approach to assessment of bleeding (Mannucci 1976), another study - Müller 2015 - used the HEmorrhage MEasurement (HEME) tool (Arnold 2007), another study - De Pietri 2016 - used the World Health Organization (WHO) bleeding score (Miller 1981), and the authors of still another study generated a scale of clinically irrelevant bleeding, minor bleeding, and major bleeding (Veelo 2012).

- The dose of FFP given to individuals in the four studies was variable. In two studies, $12 \mathrm{~mL} / \mathrm{kg}$ was used (Mannucci 1976; Müller 2015); in one study, $10 \mathrm{~mL} / \mathrm{kg}$ ideal body weight was used (De Pietri 2016); and in another study, 300 to $600 \mathrm{~mL}$ was used (dependent on prothrombin time (PT) result), equating to 4.3 to $8.6 \mathrm{~mL} / \mathrm{kg}$ for a $70-\mathrm{kg}$ individual (Veelo 2012).

- It is questionable whether the sample size for important primary outcomes including mortality and bleeding, as well as other important outcomes such as adverse events or thromboembolism, was sufficiently large to detect a difference. Lack of any events for some outcomes precluded meaningful analysis. Two of the four studies terminated recruitment early due to a slow rate of recruitment (81 participants; Müller 2015), or due to increasing resistance to FFP transfusion by physicians owing to observations of a low bleeding rate in an open-label trial (72 participants; Veelo 2012). One trial recruited 21 patients (Mannucci 1976). 
- Although three of the four studies were conducted over the last decade, one study was conducted in 1976 (Mannucci 1976). Given the development of strategies to reduce adverse events from plasma transfusion, including pathogen reduction techniques or use of male donors (Rogers 2015), how applicable these historical data remain to modern clinical practice remains questionable.

- Although one of the included studies utilised thromboelastography (De Pietri 2016), accessibility of such a modality for use in global clinical practice will depend on availability at individual institutions, which requires significant cost, equipment maintenance, quality control procedures, and user training (Subramanian 2018). Furthermore, there may be intra-operator and inter-operator variability in generated results (Quarterman 2014), although newer systems that utilise an automated process for sample and reagent mixing and activation may reduce the scope for operator-dependent variability and the requirement for training (Hartmann 2018).

\section{Quality of the evidence}

All five included studies were parallel-group randomised controlled trials (RCTs). Two were open-label trials, with high risk of both performance bias and detection bias (De Pietri 2016; Veelo 2012). One study used an open-label blinded-endpoint design with high risk of performance bias (Müller 2015). In one study, details of study design were unclear (Mannucci 1976). Another study, which ceased recruitment after enrolling two participants, was a double-blinded trial (NCT00953901). No published report is available for review of its methods or analysis of its methodological quality.

Our judgements for risk of bias of included studies can be found in the risk of bias tables in the Characteristics of included studies section of the review, and they are summarised in Figure 2 and Figure 3

- We judged three studies to be at high risk of bias in three out of seven domains (De Pietri 2016; Müller 2015; Veelo 2012).

- We judged one study to be at low risk of bias in three out of seven domains (De Pietri 2016).

- We judged two studies to be at low risk of bias in four out of seven domains (Müller 2015; Veelo 2012).

We assessed the quality of evidence using the GRADE approach, finding that quality ranged from low to very low.

With regard to the main outcomes of this review, we assessed the quality of evidence as low for the following.

- Number of individuals requiring transfusion, overall use of products within seven days, and comparison of FFP use versus different transfusion thresholds: we downgraded the evidence due to serious risk of bias and indirectness in these studies.

We assessed the quality of evidence as very low for the following.

- ICU mortality at 30 days: we downgraded the evidence for very serious risk of indirectness due to inclusion of a single trial of 76 participants, conducted in an intensive treatment unit (ITU) setting in patients undergoing a single procedure (percutaneous tracheostomy), which reported this outcome, thereby limiting applicability of study findings to the review's wider surgical setting. We also downgraded the evidence for serious risk of imprecision, given the wide confidence interval that crossed the line of no difference.

- Major bleeding within 24 hours: we downgraded the evidence due to serious risk of bias in some trials, serious or very serious risk of indirectness in some trials, and serious risk of imprecision in all trials.

- Number of transfusions per participant within seven days: we downgraded the evidence due to serious risk of bias, indirectness, and imprecision in some trials.

- Number of individuals requiring a transfusion within seven days in the setting of FFP transfusion versus no transfusion: we downgraded the evidence due to the trial's serious risk of bias, indirectness, and imprecision.

- Serious adverse events measured by plasma transfusion-related complications within 24 hours: we downgraded the evidence due to serious risk of bias in some trials, serious risk of indirectness in all trials, and serious or very serious risk of imprecision in all trials.

- Serious adverse events measured by surgery-related complications within 30 days in the setting of FFP use based on different transfusion thresholds: we downgraded the evidence due to serious risk of bias, indirectness, and imprecision in these trials.

\section{Potential biases in the review process}

This review reflects no obvious bias. A wide and comprehensive search was performed with no restriction on language, country, or publication status. We are grateful to Cochrane Russia for translation of a Russian text (Tselmakh 2008). We contacted authors of two trials for further information and are currently awaiting response (Boyd 1996; De Pietri 2016).

We used Covidence software to permit two of three review authors $(\mathrm{JH}, \mathrm{LE}, \mathrm{KW})$ to independently and carefully screen each reference, abstract, or full text for relevance. Given the large number of references initially found through the wide search (7032 results), three review authors carried out this task. Two review authors $(\mathrm{JH}$ and LE) independently extracted and agreed on data from included studies. Two review authors (PF and $\mathrm{JH}$ ) independently extracted and checked for agreement of data from ongoing studies or studies awaiting classification. Given the small number of included trials, construction of a funnel plot for identification of publication bias was not possible. In one included trial (NCT00953901), which was terminated after two participants were enrolled, no result data were published, thereby precluding assessment. Given early termination and the small patient sample size (two participants), publication bias is unlikely.

We pre-specified all reported outcomes and subgroups for analysis in advance; these were published in the review protocol before the search was conducted.

\section{Agreements and disagreements with other studies or reviews}

This review has shown lack of clear evidence demonstrating benefit for the use of prophylactic FFP before non-cardiac surgical or invasive procedures across a range of outcomes. This is consistent with two previous systematic reviews, which indicated lack of effectiveness within a range of different clinical settings (Stanworth 2004; Yang 2012). 
However this review has highlighted the uncertainty of our confidence in the evidence and in the results due to low- or predominantly very low-quality evidence for important patientcentred outcomes. This is also consistent with findings of the aforementioned systematic reviews, which found limited evidence for most clinical situations - Stanworth 2004 - and noted little improvement in methodological quality over the years - Yang 2012.

Moreover, the small number of trials pertaining to non-cardiac surgery, the large number of trials in which only a small number of participants were enrolled, and the low incidence of certain outcome events (such as bleeding or adverse events), as highlighted by previous reviews, represent a consistent theme over the past 15 years and within this review.

The limited quality of evidence in this review is also consistent with that of a recent Cochrane Review on plasma use in the alternative setting of cardiovascular surgery (Desborough 2015), which judged the quality of evidence as moderate or low for most outcomes.

\section{AUTHORS' CONCLUSIONS}

\section{Implications for practice}

Given available RCT evidence, we can neither support nor oppose the prophylactic use of FFP in non-cardiac surgery or invasive procedures over a range of clinically important outcomes. This is due to lack of confidence in the certainty of evidence and in general applicability of review findings due to an absence of highquality evidence, together with a paucity of evidence in settings such as major body cavity surgery, extensive soft tissue surgery, orthopaedic surgery, or neurosurgery.

Recent data show that inappropriate prophylactic transfusion of plasma is still commonplace (Desborough 2016b; Görlinger 2015; Lu 2017), despite more than a decade of reviews and narratives highlighting lack of evidence for this approach (Desborough 2012; Desborough 2016b; Holland 2006b; Stanworth 2004; Stanworth 2007; Verghese 2008; Weeder 2014; Yang 2012; Zakeri 2017).

Furthermore, given the global challenges of providing safe transfusion, particularly in areas of the world where testing for one or more markers of transfusion-transmissible diseases in donors is not available, and given the seven-fold difference in blood donation rates between low-income (4.6 per 1000 population per year) and high-income (32.1 per 1000 population per year) countries (WHO 2017), it is necessary to ensure that this limited resource is used effectively and appropriately.

By highlighting both the lack of high-quality evidence and the paucity of available data for the utility of prophylactic FFP transfusion, this review demonstrates uncertainty regarding its prophylactic role in clinical practice in the setting of non-cardiac surgery or invasive procedures in the absence of inherited bleeding disorders or use of anticoagulants.

\section{Implications for research}

High-quality multi-centre trials examining the safety and effectiveness of prophylactic FFP are required. These should involve participants in common major surgical operative settings, given that major body surgery was under-represented (10 participants in a single study; De Pietri 2016), and that extensive soft tissue, orthopaedic, and neurological surgeries were not represented by the available evidence. Furthermore, studies investigating paediatric participants are needed; lack of available trials for this cohort for inclusion within this review highlights the deficiency of data for this group.

Such studies would require sample sizes large enough to be adequately powered to determine differences in important patientcentred outcomes including 30-day survival, risk of major bleeding, requirement for transfusion, and risk of adverse events. Given the low incidence of bleeding and adverse events reported in this review, larger estimated sample sizes are likely required than were previously envisaged by study authors (Veelo 2012).

Furthermore, issues that may impact clinical utility, including type of plasma used (such as pathogen inactivated), post-thaw duration before infusion, and plasma dosage, would need to be considered in a study protocol. This challenge would likely be compounded by difficulties associated with recruiting large numbers of individuals owing to challenges of consent, randomisation, and enrolment of participants within a limited clinical window of opportunity, participant refusal for enrolment, or resistance of clinicians to include participants for fear of randomisation to study arms contrary to their strongly upheld beliefs (Müller 2015; Veelo 2012). Variability in FFP doses reported, together with variability in bleeding definitions and in tools used for assessment and reporting of bleeding, reflects issues that have potential implications for the ability to detect a difference between study arms and to limit the degree to which meaningful comparison of studies and meta-analysis can occur. Consistency of these issues would be valuable in future research. Future studies may also consider examining measures of quality of life. Furthermore, although research comparing FFP versus other pro-haemostatic agents has utility, these comparisons were positioned on a backdrop of poorquality evidence when FFP was compared with no FFP.

\section{ACK N O WLEDGEMENTS}

We thank the following study authors for their response following correspondence for additional information: G Boyd, D Veelo.

We thank the editorial base of the Cochrane Haematological Malignancies Review Group.

We thank peer and consumer reviewers of this protocol.

We thank NHS Blood and Transplant.

We thank the National Institute of Health Research (NIHR). This review is part of a series of reviews that have been funded by the NIHR Cochrane Programme Grant - Safe and Appropriate Use of Blood Components. This research was also supported by the NIHR Oxford Biomedical Research Centre Programme. The views expressed are those of the authors and not necessarily those of the NHS, the NIHR, or the Department of Health. 


\section{R E F E R E N C E S}

\section{References to studies included in this review}

De Pietri 2016 \{published data only\}

De Pietri L. Thromboelastographic guide for blood products in cirrhotics. clinicaltrials.gov/ct2/show/NCT02362178 (first received February 2015). [NCT02362178]

* De Pietri L, Bianchini M, Montalti R, De Maria N, Di Maira T, Begliomin B, et al. Thrombelastography-guided blood product use before invasive procedures in cirrhosis with severe coagulopathy: a randomized, controlled trial. Hepatology 2016;63(2):566-73. [DOI: https://dx.doi.org/10.1002/hep.28148]

De Pietri L, Bianchini M, Montalti R, Maria N, Maira T, Begliomini B, et al. Thrombelastography (TEG) decreases blood products requirement before invasive procedures in cirrhotic patients with coagulation tests derangement. A randomized controlled trial. Digestive and Liver Disease 2014; Vol. 46:e5-6.

\section{Mannucci 1976 \{published data only\}}

Mannucci PM, Franchi F, Dioguardi N. Correction of abnormal coagulation in chronic liver disease by combined use of freshfrozen plasma and prothrombin complex concentrates. Lancet 1976;2 (7985):542-5.

\section{Müller 2015 \{published data only\}}

Juffermans NP. Transfusion of fresh frozen plasma in nonbleeding intensive care unit (ICU) patients. clinicaltrials.gov/ show/NCT01143909\%202010 (first received June 2010). [NCT01143909]

* Muller MC, Arbous MS, Spoelstra-de Man AM, Vink R, Karakus A, Straat M, et al. Transfusion of fresh-frozen plasma in critically ill patients with a coagulopathy before invasive procedures: a randomized clinical trial (CME). Transfusion 2015;55(1):26-35; quiz 25. [DOI: 10.1111/trf.12750]

Muller MC, Juffermans NP. Transfusion of plasma to prevent bleeding in ICU patients [Transfusion of fresh frozen plasma in non-bleeding ICU patients]. trialregister.nl/trialreg/admin/ rctview.asp?TC=2262 (first received March 2010). [NTR2262]

Müller MC, de Jonge E, Arbous MS, Spoelstra-de Man AM, Karakus A, Vroom MB, et al. Transfusion of fresh frozen plasma in non-bleeding ICU patients - TOPIC trial: study protocol for a randomized controlled trial. Trials 2011;12:266. [DOI: 10.1186/1745-6215-12-266]

Straat M, Müller MC, Meijers JC, Arbous MS, Spoelstra-de Man AM, Beurskens CJ, et al. Effect of transfusion of fresh frozen plasma on parameters of endothelial condition and inflammatory status in non-bleeding critically ill patients: a prospective substudy of a randomized trial. Critical Care 2015;19(1):163. [DOI: 10.1186/s13054-015-0828-6]

\section{NCT00953901 \{published data only\}}

Mayo Clinic. Is plasma transfusion beneficial prior to low-risk procedures in hospitalized patients with blood clotting abnormalities?. clinicaltrials.gov/ct2/show/record/ NCT00953901 (first received August 2009). [NCT00953901]
Veelo 2012 \{published data only\}

Veelo DP, Vlaar AP, Dongelmans DA, Binnekade JM, Levi M, Paulus F, et al. Correction of subclinical coagulation disorders before percutaneous dilatational tracheotomy. Blood Transfusion 2012;10(2):213-20. [DOI: 10.2450/2012.0086-11]

\section{References to studies excluded from this review}

Bauer 1986 \{published data only\}

Bauer HW, Schmiedt E. Prophylaxis of complications after urological abdominal surgery by substitution of plasma proteins. Journal of Surgical Oncology 1986;31(3):174-7.

\section{Cao 2016 \{published data only\}}

Cao X, Zhang X, Li Q. [Efficacy of thromboelastography to monitor the clinical massive transfusion in scoliosis: a randomized controlled trial]. Zhonghua Wai Ke Za Zh 2016;54(2):137-41. [DOI: 10.3760/ cma.j.issn.0529-5815.2016.02.013]

\section{ChiCTR-INR-17013901 \{published data only\}}

ChiCTR-INR-17013901. The effect of low-dose, early FFP transfusion on early complications and long-term outcomes of patients with severe trauma brain injury. chictr.org.cn/ showproj.aspx?proj=23901 (first received December 2017).

\section{Freeman 1998 \{published data only\}}

Freeman JW, Williamson LM, Llewelyn C, Fisher N, Allain JP, Bellamy $\mathrm{M}$, et al. A randomized trial of solvent/detergent and standard fresh frozen plasma in the treatment of the coagulopathy seen during orthotopic liver transplantation. Vox Sanguinis 1998;74(Suppl 1):2.

* Freeman JW, Williamson LM, Llewelyn C, Fisher N, Allain JP, Bellamy $\mathrm{M}$, et al. A randomized trial of solvent/detergent and standard fresh frozen plasma in the treatment of the coagulopathy seen during orthotopic liver transplantation. Vox Sanguinis 1998;74(Suppl 1):225-9.

Galganski 2017 \{published data only\}

* Galganski LA, Greenhalgh DG, Sen S, Palmieri TL. Randomized comparison of packed red blood cell-to-fresh frozen plasma transfusion ratio of $4: 1$ vs $1: 1$ during acute massive burn excision. Journal of Burn Care \& Research 2017;38(3):194-201. [DOI: 10.1097/bcr.0000000000000468]

Galganski LA, Sen S, Greenhalgh DG, Palmieri TI. Randomized comparison of packed red blood cell-to-fresh frozen plasma transfusion ratio of $4: 1$ versus $1: 1$ during acute massive burn excision. Journal of Burn Care \& Research 2016;37:S109.

Gazzard 1975 \{published data only\}

Gazzard BG, Henderson JM, Williams R. The use of fresh frozen plasma or a concentrate of factor IX as replacement therapy before liver biopsy. Gut 1975;16(8):621-5.

\section{Hedstrand 1987 \{published data only\}}

Hedstrand U, Högman C, Zarén B, Lundkvist B. Postoperative complications after blood replacement with or without

Prophylactic plasma transfusion for patients without inherited bleeding disorders or anticoagulant use undergoing non-cardiac surgery 
plasma. A trial in elective surgery. Acta Chirurgica Scandinavica 1987;153(9):501-5.

\section{Hildebrandt 2007 \{published data only\}}

Hildebrandt B, Machotta A, Riess H, Kerner S, Ahlers O, Haberl H, et al. Intraoperative fresh-frozen plasma versus human albumin in craniofacial surgery - a pilot study comparing coagulation profiles in infants younger than 12 months. Thrombosis and Haemostasis 2007;98(1):172-7.

\section{Jilma-Stohlawetz 2011 \{published data only\}}

Jilma P, Horvath M, Leitner G, List J, Derhaschnig U, Kursten FW, et al. Safety of a universal plasma: a randomized, double blind, active controlled clinical trial. Transfusion 2010;50(Suppl 2):61A.

Jilma P, Horvath M, Leitner G, List J, Walasek C, Kursten F. Safety of a universal plasma (UNIPLAS): a randomized, double-blind, active controlled clinical trial. Vox Sanguinis 2010;99(Suppl S1):235-6.

* Jilma-Stohlawetz P, Kursten FW, Walasek C, Horvath M, Leitner G, List J, et al. Safety of a universal, virus-inactivated and prion-depleted, pharmaceutical-quality plasma: a randomized, double-blind, clinical trial in healthy volunteers. Transfusion 2011;51(6):1228-40.

\section{Kerner 2008 \{published data only\}}

Kerner T, Machotta A, Kerner S, Ahlers O, Haberl H, Riess H, et al. A clinical pilot study of fresh frozen plasma versus human albumin in paediatric craniofacial repair. Journal of International Medical Research 2008;36(1):171-7.

\section{Laine 2003 \{published data only\}}

Laine E, Steadman R, Calhoun L, Blackall D, Levin P, Braunfeld $\mathrm{M}$, et al. Comparison of RBCs and FFP with whole blood during liver transplant surgery. Transfusion 2003;43(3):322-7.

\section{Lance 2012 \{published data only\}}

Lancé MD, Ninivaggi M, Hamulyak K, van Pampus ECM, ten Cate $\mathrm{H}$, Heemskerk JWM. Early transfusion with FFP or FFP plus fibrinogen concentrate in massive hemorrhage: a randomized intervention trial: 6AP5-5. European Journal of Anaesthesiology 2011;28:92.

Lancé MD, Ninivaggi M, Marcus MAE, Hamulyak K, van Pampus ECM, ten Cate $\mathrm{H}$, et al. Partial prohemostatic effect of transfusion of fibrinogen concentrate replacing fresh frozen plasma in massive hemorrhage. Journal of Thrombosis and Haemostasis 2011;9:229. [DOI: 10.1111/ j.1538-7836.2011.04380_1.x]

* Lancé MD, Ninivaggi M, Schols SEM, Feijge MA, Oehrl SK, Kuiper GJAJM. Perioperative dilutional coagulopathy treated with fresh frozen plasma and fibrinogen concentrate: a prospective randomized intervention trial. Vox Sanguinis 2012;103(1):25-34. [DOI: 10.1111/j.1423-0410.2011.01575.x]

\section{Lerner 1997 \{published data only\}}

Lerner R, Scorcia E, Pehta J, Zarou C, Bes J, et al. Solvent detergent-treated plasma versus FFP in patients with prolonged prothrombin time. Transfusion 1997;37(9S):22S.

\section{Liu 1994 \{published data only\}}

Liu YC, Ho WM, Kang HM, Tso HS. Observation of coagulation change during induced hypotensive anesthesia and autologous plasma transfusion. Acta Anaesthesiologica Sinica 1994;32(3):159-64.

\section{Mintz 2006 \{published data only\}}

Corash L, Bass N, Petz L, Steadman R, Streif M, McCullough J, et al. Fresh frozen plasma for management of hemostasis during minor and major surgical procedures associated with coagulopathy. Vox Sanguinis 2006;91(Suppl 3):185.

Mintz P, Steadman R, Blackall D, Petz L, McCullough J, Bass N, et al. INTERCEPT plasma using Helinx(R) technology is well tolerated in the treatment of end-stage liver disease patients - the STEP AC trial. 7th Annual Meeting of the European Haematology Association. 2002:87-92.

* Mintz PD, Bass NM, Petz LD, Steadman R, Streiff M, McCullough J, et al. Photochemically treated fresh frozen plasma for transfusion of patients with acquired coagulopathy of liver disease. Blood 2006;107(9):3753-60. [DOI: 10.1182/ blood-2004-03-0930]

Ramies DA, Mintz P, Steadman R, Blackall D, Petz L, Bass N, et al. INTERCEPT plasma using Helinx technology is well tolerated in the treatment of end-stage liver disease patients the STEP AC trial. Haematologica 2002;87(Suppl 1):118.

\section{NCT00233246 \{published data only\}}

NCT00233246. Fresh frozen plasma infusions to reduce risk of bleeding related to invasive procedures. clinicaltrials.gov/ct2/ show/NCT00233246 (first received October 2005).

\section{NCT00235183 \{published data only\}}

NCT00235183. Plasma transfusion in liver transplantation. clinicaltrials.gov/ct2/show/NCT00235183 (first received October 2005).

\section{NCT00302965 \{published data only\}}

NCT00302965. Effectiveness of plasma transfusions in critical care patients. clinicaltrials.gov/beta/show/NCT00302965? term=NCT00302965\&rank=1 (first received March 2006).

\section{NCT00656396 \{published data only\}}

NCT00656396. Point of care coagulation testing in patients undergoing major surgery. clinicaltrials.gov/ct2/show/record/ NCT00656396 (first received April 2008).

\section{NCT00994045 \{published data only\}}

NCT00994045. Fibrinogen as an alternative to FFP in aortic surgery. clinicaltrials.gov/ct2/show/record/NCT00994045 (first received October 2009). [EudraCT Number: 2009-016709-41]

\section{NCT02352181 \{published data only\}}

NCT02352181. Management of coagulopathy during orthotopic liver transplantation. Comparison between ROTEM-based management and standard biological assessment. clinicaltrials.gov/ct2/show/NCT02352181? term=NCT02352181\&rank=1 (first received January 2015). 


\section{NCT03700723 \{published data only\}}

NCT03700723. Comparison of the efficacy and safety of Resusix with FP24 in patients with acquired coagulopathy. clinicaltrials.gov/ct2/show/NCT03700723. Study Status: Recruiting: Prospectively registered? Yes, (first received October 2018).

\section{Palmieri 2013 \{published data only\}}

Palmieri TL, Greenhalgh DG, Sen S. Prospective comparison of packed red blood cell-to-fresh frozen plasma transfusion ratio of 4:1 versus $1: 1$ during acute massive burn excision. Journal of Trauma and Acute Care Surgery 2013;74(1):76-83. [DOI: 10.1097/ TA.0b013e31827891b7]

\section{Pieters 2015 \{published data only\}}

Pieters BJ, Conley L, Weiford J, Hamilton M, Wicklund B, Booser $A$, et al. Prophylactic versus reactive transfusion of thawed plasma in patients undergoing surgical repair of craniosynostosis: a randomized clinical trial. Pediatric Anesthesia 2015;25(3):279-87. [DOI: 10.1111/pan.12571]

\section{Ramies 2002 \{published data only\}}

Ramies D, Benjamin R, Wages D, Valentine M, Ziomek Z, Corash $\mathrm{L}$. The STEP trials demonstrate the safety of INTERCEPT treated fresh frozen plasma. Vox Sanguinis 2002;83(Suppl 2):118.

\section{Rocha 2017 \{published data only\}}

Rocha L, Pessoa CM, Neto AS, do Prado RR, Silva E, de Almeida MD, et al. Thromboelastometry versus standard coagulation tests versus restrictive protocol to guide blood transfusion prior to central venous catheterization in cirrhosis: study protocol for a randomized controlled trial. Trials 2017;18(1):85. [DOI: 10.1186/s13063-017-1835-5]

\section{Sommoggy 1990 \{published data only\}}

Sommoggy ST, Fraunhofer J, Jelen-Esselborn Stemberger SA. Coagulation changes during aortofemoral bifurcation bypass operations: is plasma substitution possible with hydroxyethyl starch (HES) only?. Anaesthesist 1990;39(7):353-360.

\section{Tinmouth 2008 \{published data only\}}

Tinmouth A, Chatelain E, Fergusson, McIntyre L, Giulivi A, Hubert. A randomized controlled trial of high and standard dose frozen plasma transfusions in critically ill patients. Transfusion 2008;48(Suppl 2):26A-27A.

\section{Urwyler 2009 \{published data only\}}

Urwyler N1, Trelle S, Theiler L, Jüni P, Staub LP, Luyet C, et al. Does point of care prothrombin time measurement reduce the transfusion of fresh frozen plasma in patients undergoing major surgery? The POC-OP randomized-controlled trial. Trials 2009;23(10):107. [DOI: 10.1186/1745-6215-10-107]

\section{Wang 2010 \{published data only\}}

Wang SC, Shieh JF, Chang KY, Chu YC, Liu CS, Loong CC, et al. Thromboelastography-guided transfusion decreases intraoperative blood transfusion during orthotopic liver transplantation: randomized clinical trial. Transplantation Proceedings 2010;42(7):2590-3. [DOI: 10.1016/ j.transproceed.2010.05.144]

\section{Wieding 1999 \{published data only\}}

Wieding J, Rathgebe J, Zenker D, Pohl U, Koehler M. Prospective, randomized and controlled study on solvent/ detergent versus methylene blue/light virus-inactivated plasma. Transfusion 1999;39(S10):23S.

Williamson 1999 \{published data only\} Williamson LM, Llewelyn CA, Fisher NC, Allain JP, Bellamy MC, Baglin TP, et al. A randomized trial of solvent/detergenttreated and standard fresh-frozen plasma in the coagulopathy of liver disease and liver transplantation. Transfusion 1999;39(11-12):1227-34.

\section{References to studies awaiting assessment}

Boyd 1996 \{published data only\}

Boyd GL, Diethelm AG, Gelman S, Langner R, Laskow D, Deierhoi $\mathrm{M}$, et al. Correcting prolonged bleeding during renal transplantation with estrogen or plasma. Archives of Surgery 1996;131:160-5.

\section{NCT02777424 \{published data only\}}

NCT02777424. Prothrombin complex concentrate versus fresh frozen plasma to correct coagulation disorders in adult neurosurgical patients (CLOT-CRANE). clinicaltrials.gov/ ct2/show/study/NCT02777424 (first received May 2016). [AGR_2014-22]

\section{Tseĭmakh 2008 \{published data only\}}

Tsermakh EA, Bombizo VA, Buldakov PN, Siniavin AV, Osipov ES, Meliksetian AD, et al. [The use of cryosupernatant plasma in complex treatment of pancreonecrosis]. Khirurgiia 2008;8:32-7.

\section{References to ongoing studies}

NCT02561026 \{published data only\}

NCT02561026. Transfusion of plasma prior to invasive procedures pilot trial (TOPPIT). clinicaltrials.gov/ct2/show/ study/NCT02561026 (first received September 2015).

\section{NCT02637427 \{published data only\}}

NCT02637427. Does plasma reduce bleeding in patients undergoing invasive procedures. clinicaltrials.gov/ct2/ show/NCT02637427 (first received December 2015). [1R34HL125804-01A1 (U.S. NIH Grant/Contract)]

\section{Smart 2017 \{published data only\}}

Smart L, Wellner M, Gray N, Michaels A, Kirkpatrick R, Conteh L, et al. A prospective, randomised clinical trial comparing blood product use, bleeding events, and cost during and after endoscopic procedures in patients with cirrhosis and coagulopathy: rotational thromboelastometry (ROTEM) versus conventional therapy. Hepatology 2017;66(Suppl 1):243A-4A.

Prophylactic plasma transfusion for patients without inherited bleeding disorders or anticoagulant use undergoing non-cardiac surgery 


\section{Additional references}

\section{Abdel-Wahab 2006}

Abdel-Wahab OI, Healy B, Dzik WH. Effect of fresh-frozen plasma transfusion on prothrombin time and bleeding in patients with mild coagulation abnormalities. Transfusion 2006;46(8):1279-85.

\section{Abolghasemi 2010}

Abolghasemi H, Hosseini-Divkalayi NS, Seighali F. Blood donor incentives: a step forward or backward. Asian Journal of Transfusion Science 2010;4(1):9-13.

\section{ACS 2007}

American College of Surgeons. Statement on surgery using lasers, pulsed light, radiofrequency devices, or other techniques. Bulletin of the American College of Surgeons April 2007;92(4):37-8.

\section{American Society of Anesthesiologists 2006}

American Society of Anesthesiologists. Practice guidelines for perioperative blood transfusion and adjuvant therapies: an updated report by the American Society of Anesthesiologists Task Force on Perioperative Blood Transfusion and Adjuvant Therapies. Anesthesiology 2006;105(1):198-208.

\section{American Society of Anesthesiologists 2015}

American Society of Anesthesiologists. Practice guidelines for perioperative blood management: an updated report by the American Society of Anesthesiologists Task Force on Perioperative Blood Management. Anesthesiology 2015;122(2):241-75.

\section{Arnold 2007}

Arnold DM, Donahoe L, Clarke FJ, Tkaczyk AJ, Heels-Ansdell D, Zytaruk N, et al. Bleeding during critical illness: a prospective cohort study using a new measurement tool. Clinical and Investigative Medicine 2007;30(2):E93-E102.

\section{Benjamin 2012}

Benjamin RJ, McLaughlin LS. Plasma components: properties, differences, and uses. Transfusion 2012;52 Suppl 1:9s-19s.

\section{Chee 2008}

Chee YL, Crawford JC, Watson HG, Greaves M. Guidelines on the assessment of bleeding risk prior to surgery or invasive procedures. British Committee for Standards in Haematology. British Journal of Haematology 2008;140(5):496-504.

\section{Chou 2010}

Chou R, Aronson N, Atkins D, Ismaila AS, Santaguida P, Smith $\mathrm{DH}$, et al. AHRQ series paper 4: assessing harms when comparing medical interventions: AHRQ and the effective health-care program. Journal of Clinical Epidemiology 2010; Vol. 63, issue 5:502-12.

\section{Covidence 2016 [Computer program]}

Veritas Health Innovation. Covidence systematic review software. Version accessed 5 February 2017. Melbourne: Veritas Health Innovation, 2016.

\section{Darcy 1996}

Darcy MD, Kanterman RY, Kleinhoffer MA, Vesely TM, Picus D, Hicks ME, et al. Evaluation of coagulation tests as predictors of angiographic bleeding complications. Radiology 1996;198(3):741-4

\section{Deeks 2011}

Deeks JJ, Higgins JPT, Altman DG (editors). Chapter 9. Analysing data and undertaking meta-analyses. In: Higgins J P T, Green S, editor(s). Cochrane Handbook for Systematic Reviews of Interventions Version 5.1.0 (updated March 2011). The Cochrane Collaboration, 2011. Available from www.handbook.cochrane.org.

\section{Deitcher 2002}

Deitcher SR. Interpretation of the international normalised ratio in patients with liver disease. Lancet 2002;359(9300):47-8.

\section{Desborough 2012}

Desborough M, Stanworth SJ. Plasma transfusion for bedside, radiologically guided, and operating room invasive procedures. Transfusion 2012;52 Suppl 1:20s-9s.

\section{Desborough 2015}

Desborough M, Sandu R, Brunskill SJ, Doree C, Trivella M, Montedori A, et al. Fresh frozen plasma for cardiovascular surgery. Cochrane Database of Systematic Reviews 2015, Issue 7. [DOI: 10.1002/14651858.CD007614.pub2]

\section{Desborough 2016a}

Desborough M, Hadjinicolaou AV, Chaimani A, Trivella M, Vyas $\mathrm{P}$, Doree $\mathrm{C}$, et al. Alternative agents to prophylactic platelet transfusion for preventing bleeding in people with thrombocytopenia due to chronic bone marrow failure: a meta-analysis and systematic review. Cochrane Database of Systematic Reviews 2016, Issue 10. [DOI: 10.1002/14651858.CD012055.pub2]

\section{Desborough 2016b}

Desborough MJ, Hockley B, Sekhar M, Burroughs AK, Stanworth SJ, Jairath V, et al. Patterns of blood component use in cirrhosis: a nationwide study. Liver International 2016;36(4):522-9.

\section{Dhingra 2013}

Dhingra N. International challenges of self-sufficiency in blood products. Transfusion Clinique et Biologique 2013;20(2):148-52.

\section{Dzik 2004}

Dzik WH. Predicting hemorrhage using preoperative coagulation screening assays. Current Hematology Reports 2004;3(5):324-30.

\section{Eder 2007}

Eder AF, Herron R, Strupp A, Dy B, Notari EP, Chambers LA, et al. Transfusion-related acute lung injury surveillance (2003-2005) and the potential impact of the selective use of plasma from male donors in the American Red Cross. Transfusion 2007;47(4):599-607. 


\section{Ejaz 2015}

Ejaz A, Frank SM, Spolverato G, Kim Y, Pawlik TM. Defining transfusion triggers and utilization of fresh frozen plasma and platelets among patients undergoing hepatopancreaticobiliary and colorectal surgery. Annals of Surgery 2015;262(6):1079-85.

\section{Glance 2011}

Glance LG, Dick AW, Mukamel DB, Fleming FJ, Zollo RA, Wissler R, et al. Association between intraoperative blood transfusion and mortality and morbidity in patients undergoing noncardiac surgery. Anesthesiology 2011;114(2):283-92.

\section{GRADEpro 2015 [Computer program]}

McMaster University (developed by Evidence Prime, Inc). GRADEproGDT: GRADEpro Guideline Development Tool. Version accessed 5 February 2017. McMaster University (developed by Evidence Prime, Inc), 2015.

\section{Görlinger 2015}

Görlinger K, Saner FH. Prophylactic plasma and platelet transfusion in the critically ill patient: just useless and expensive or even harmful?. BMC Anesthesiology 2015;15:86. [DOI: 10.1186/s12871-015-0074-0]

\section{Hall 2016}

Hall DP, Estcourt LJ, Doree C, Hopewell S, Trivella M, Walsh TS. Plasma transfusions prior to insertion of central lines for people with abnormal coagulation. Cochrane Database of Systematic Reviews 2016, Issue 9. [DOI: 10.1002/14651858.CD011756.pub2]

\section{Hartmann 2018}

Hartmann J, Mason D, Achneck H. Thromboelastography (TEG) point-of-pare diagnostic for hemostasis management. Point of Care 2018;17(1):15-22.

\section{Higgins 2011a}

Higgins JPT, Deeks JJ, editors. Chapter 7. Selecting studies and collecting data. In: Higgins JPT, Green S, editors. Cochrane Handbook for Systematic Reviews of Interventions Version 5.1.0 (updated March 2011). The Cochrane Collaboration, 2011. Available from www.handbook.cochrane.org.

\section{Higgins 2011b}

Higgins JPT, Altman DG, Sterne JAC, editors. Chapter 8. Assessing risk of bias in included studies. In: Higgins JPT, Green S, editors. Cochrane Handbook for Systematic Reviews of Interventions Version 5.1.0 (updated March 2011). The Cochrane Collaboration, 2011. Available from www.handbook.cochrane.org.

\section{Higgins 2011c}

Higgins JPT, Deeks JJ, Altman DG, editors. Chapter 16. Special topics in statistics. In: Higgins JPT, Green S, editors. Cochrane Handbook for Systematic Reviews of Interventions Version 5.1.0 (updated March 2011). The Cochrane Collaboration, 2011. Available from www.handbook.cochrane.org.

\section{Holland 2006a}

Holland LL, Brooks JP. Toward rational fresh frozen plasma transfusion: the effect of plasma transfusion on coagulation test results. American Journal of Clinical Pathology 2006;126(1):133-9.

\section{Holland 2006b}

Holland LL, Sarode R. Should plasma be transfused prophylactically before invasive procedures?. Current Opinion in Hematology 2006;13(6):447-51.

\section{Holness 2004}

Holness L, Knippen MA, Simmons L, Lachenbruch PA. Fatalities caused by TRALI. Transfusion Medicine Reviews 2004;18(3):184-8.

\section{HPRA 2018}

Health Products Regulatory Authority (HPRA), Ireland. hpra.ie/img/uploaded/swedocuments/Product\%20Leaflet \%20Approved-2143463-03112014151211-635506243323775000.pdf (accessed 13 April 2018).

\section{Hunt 2014}

Hunt BJ. Bleeding and coagulopathies in critical care. New England Journal of Medicine 2014;370(22):2153.

\section{ICD9-CM 2011}

National Center for Health Statistics (NCHS) and Centers for Disease Control and Prevention (CDC). International Classification of Diseases, ninth revision, clinical modification (ICD-9-CM). www.cdc.gov/nchs/icd/icd9cm.htm. 2011 (accessed 27 December 2017).

\section{ICH 1994}

International Conference on Harmonisation (ICH) of Technical Requirements for Registration of Pharmaceuticals for Human Use. ICH Harmonised Tripartite Guideline. Clinical Data Safety Management: Definitions and Standards for Expedited Reporting E2A, 1994. Available from www.ich.org/fileadmin/ Public_Web_Site/ICH_Products/Guidelines/Efficacy/E2A/Step4/ E2A_Guideline.pdf (accessed 5 February 2017).

\section{ISBT 2011}

International Society of Blood Transfusion (ISBT) Working Party on Haemovigilance. Proposed standard definitions for surveillance of non infectious adverse transfusion reactions, 2011. Available from inn-org.com/wp-content/ uploads/2011/06/ISBT-definitions-for-non-infectioustransfusion-reactions.pdf (accessed 5 February 2017).

\section{Karam 2013}

Karam O, Lacroix J, Robitaille N, Rimensberger PC, Tucci M. Association between plasma transfusions and clinical outcome in critically ill children: a prospective observational study. Vox Sanguinis 2013;104(4):342-9.

\section{Khan 2007}

Khan H, Belsher J, Yilmaz M, Afessa B, Winters JL, Moore SB, et al. Fresh-frozen plasma and platelet transfusions are associated with development of acute lung injury in critically ill medical patients. Chest 2007;131(5):1308-14. 


\section{Kinard 2013}

Kinard NT, Christie A, Greilich PE, Sarode R. Comparison of thromboelastography (TEG) with rotational thromboelastometry (ROTEM) in surgical patients. Blood 2013;122(21):3659.

\section{Kozar 2011}

Kozar RA, Peng Z, Zhang R, Holcomb JB, Pati S, Park P, et al. Plasma restoration of endothelial glycocalyx in a rodent model of hemorrhagic shock. Anesthesia \& Analgesia 2011;112(6):1289-95.

\section{Liberati 2009}

Liberati A, Altman DG, Tetzlaff J, Mulrow C, Gotzsche PC, loannidis JP, et al. The PRISMA statement for reporting systematic reviews and meta-analyses of studies that evaluate health care interventions: explanation and elaboration. PLOS Medicine 2009;6(7):e1000100.

\section{Lira 2014}

Lira A, Pinsky MR. Choices in fluid type and volume during resuscitation: impact on patient outcomes. Annals of Intensive Care 2014;4:38.

\section{Liumbruno 2009}

Liumbruno G, Bennardello F, Lattanzio A, Piccoli P, Rossetti G. Recommendations for the transfusion of plasma and platelets. Blood Transfusion 2009;7(2):132-50.

\section{Lu 2017}

Lu Y, Sun G, Liu X, Liu Z, Tan J, Hao Y, et al. Plasma transfusion in patients with cirrhosis in China: a retrospective multicenter cohort study. Transfusion Medicine Reviews 2017;31(2):107-12.

\section{Luk 2002}

Luk C, Eckert KM, Barr RM, Chin-Yee IH. Prospective audit of the use of fresh-frozen plasma, based on Canadian Medical Association transfusion guidelines. Canadian Medical Association Journal 2002;166(12):1539-40.

\section{MacLennan 2006}

MacLennan S, Williamson LM. Risks of fresh frozen plasma and platelets. Journal of Trauma Injury Infection and Critical Care 2006;60(6 Suppl):S46-50.

\section{Martlew 2000}

Martlew VJ. Peri-operative management of patients with coagulation disorders. British Journal of Anaesthesia 2000;85(3):446-55.

\section{McGill 1990}

McGill DB, Rakela J, Zinsmeister AR, Ott BJ. A 21-year experience with major hemorrhage after percutaneous liver biopsy. Gastroenterology 1990;99(5):1396-400.

\section{McGilvray 2001}

McGilvray ID, Rotstein OD. Assessment of coagulation in surgical critical care patients. In: Holzheimer RG, Mannick JA editor(s). Surgical Treatment: Evidence-Based and Problem-Oriented. Munich: Zuckschwerdt, 2001.

\section{McVay 1990}

McVay PA, Toy PT. Lack of increased bleeding after liver biopsy in patients with mild hemostatic abnormalities. American Journal of Clinical Pathology 1990;94(6):747-53.

\section{McVay 1991}

McVay PA, Toy PT. Lack of increased bleeding after paracentesis and thoracentesis in patients with mild coagulation abnormalities. Transfusion 1991;31(2):164-71.

\section{Miller 1981}

Miller AB, Hoogstraten B, Staquet M, Winkler A. Reporting results of cancer treatment. Cancer 1981;47(1):207-14.

\section{Moylan 2008}

Moylan S, Szabo F, Scott H, Kwok G. Use of fresh-frozen plasma at Royal Darwin Hospital: a retrospective audit. Internal Medicine Journal 2008;38(9):686-91.

\section{Narick 2012}

Narick C, Triulzi DJ, Yazer MH. Transfusion-associated circulatory overload after plasma transfusion. Transfusion 2012;52(1):160-5.

\section{National Blood Authority 2012}

National Blood Authority. Patient Blood Management Guidelines: Module 2 - Perioperative. Canberra ACT: National Blood Authority, 2012.

\section{NICE 2015}

National Institute for Health and Care Excellence (NICE). Blood Transfusion: NICE Guideline [NG24]. London: National Institute for Health and Care Excellence (UK), 2015.

\section{Norfolk 2013}

Norfolk D. Handbook of Transfusion Medicine. Norwich: TSO, 2013.

\section{O'Shaughnessy 2004}

O'Shaughnessy DF, Atterbury C, Bolton Maggs P, Murphy M, Thomas D, Yates $S$, et al. Guidelines for the use of fresh-frozen plasma, cryoprecipitate and cryosupernatant. British Journal of Haematology 2004;126(1):11-28.

\section{Padhi 2015}

Padhi S, Kemmis-Betty S, Rajesh S, Hill J, Murphy MF. Blood transfusion: summary of NICE guidance. BMJ 2015;351:h5832.

\section{Pahuja 2012}

Pahuja S, Sethi N, Singh S, Sharma S, Jain M, Kushwaha S. Concurrent audit of fresh frozen plasma: experience of a tertiary care hospital. Hematology 2012;17(5):306-10.

\section{Palo 2006}

Palo R, Capraro L, Hovilehto S, Koivuranta M, Krusius T, Loponen E, et al. Population-based audit of fresh-frozen plasma transfusion practices. Transfusion 2006;46(11):1921-5.

\section{Pamphilon 2000}

Pamphilon D. Viral inactivation of fresh frozen plasma. British Journal of Haematology 2000;109(4):680-93. 


\section{Parmar 1998}

Parmar MKB, Torri V, Stewart L. Extracting summary statistics to perform meta-analyses of the published literature for survival endpoints. Statistics in Medicine 1998;17:2815-34.

\section{Peng 2013}

Peng Z, Pati S, Potter D, Brown R, Holcomb JB, Grill R, et al. Fresh frozen plasma lessens pulmonary endothelial inflammation and hyperpermeability after hemorrhagic shock and is associated with loss of syndecan 1. Shock 2013;40(3):195-202.

\section{Potter 2015}

Potter DR, Baimukanova G, Keating SM, Deng X, Chu JA, Gibb SL, et al. Fresh frozen plasma and spray-dried plasma mitigate pulmonary vascular permeability and inflammation in hemorrhagic shock. Journal of Trauma and Acute Care Surgery 2015;78(6 Suppl 1):S7-s17.

\section{Prathiba 2001}

Prathiba R, Jayaranee S, Ramesh JC, Lopez CG, Vasanthi N. An audit of fresh frozen plasma usage in a tertiary referral centre in a developing country. Malaysian Journal of Pathology 2001;23(1):41-6.

\section{Puchalski 2013}

Puchalski JT, Argento AC, Murphy TE, Araujo KL, Pisani MA. The safety of thoracentesis in patients with uncorrected bleeding risk. Annals of the American Thoracic Society 2013;10(4):336-41.

\section{Quarterman 2014}

Quarterman C, Shaw M, Johnson I, Agarwal S. Intra- and intercentre standardisation of thromboelastography (TEG(R)). Anaesthesia 2014;69(8):883-90.

\section{Rahbar 2015}

Rahbar E, Cardenas JC, Baimukanova G, Usadi B, Bruhn R, Pati $S$, et al. Endothelial glycocalyx shedding and vascular permeability in severely injured trauma patients. Journal of Translational Medicine 2015;13:117.

\section{Rand 2005}

Rand JH, Senzel L. Laboratory evaluation of hemostatic disorders. In: Hoffman R, Benz EJ, Shattil SJ, Furie B, Cohen HJ, Silberstein LE, McGlave P editor(s). Hematology: Basic Principles and Practice. Philadelphia: Elsevier Churchill Livingstone, 2005.

\section{RevMan 2014 [Computer program]}

The Cochrane Collaboration. Review Manager 5 (RevMan 5). Version 5.3. Copenhagen: Nordic Cochrane Centre, The Cochrane Collaboration, 2014.

\section{Roback 2010}

Roback JD, Caldwell S, Carson J, Davenport R, Drew MJ, Eder A, et al. Evidence-based practice guidelines for plasma transfusion. Transfusion 2010;50(6):1227-39.

\section{Rogers 2015}

Rogers TS, Fung MK, Harm SK. Recent advances in preventing adverse reactions to transfusion. F1000Research 2015;4(F1000 Faculty Reviews):1469. [DOI: 10.12688/f1000research.7048.1]

\section{Rutherford 2008}

Rutherford E, Brecher M, Fakhry S, Sheldon G. Hematologic principles in surgery. In: Townsend CM, Beauchamp RD, Evers BM, Mattox KL editor(s). Sabiston Textbook of Surgery: The Biological Basis of Modern Surgical Practice. Philadelphia: Saunders/Elsevier, 2008.

\section{Sarani 2008}

Sarani B, Dunkman WJ, Dean L, Sonnad S, Rohrbach JI, Gracias VH. Transfusion of fresh frozen plasma in critically ill surgical patients is associated with an increased risk of infection. Critical Care Medicine 2008;36(4):1114-8.

\section{Schott 2016}

Schott U, Solomon C, Fries D, Bentzer P. The endothelial glycocalyx and its disruption, protection and regeneration: a narrative review. Scandinavian Journal of Trauma Resuscitation \& Emergency Medicine 2016;24:48.

\section{Schramm 2001}

Schramm B, Leslie K, Myles PS, Hogan CJ. Coagulation studies in preoperative neurosurgical patients. Anaesthesia and Intensive Care 2001;29(4):388-92.

\section{Schulman 2010}

Schulman S, Angeras U, Bergqvist D, Eriksson B, Lassen MR, Fisher W. Definition of major bleeding in clinical investigations of antihemostatic medicinal products in surgical patients. Journal of Thrombosis and Haemostasis 2010;8(1):202-4.

\section{Schünemann 2011a}

Schünemann HJ, Oxman AD, Higgins JPT, Vist GE, Glasziou P, Guyatt GH. Chapter 11. Presenting results and 'Summary of findings' tables. In: Higgins JPT, Green S, editor(s). Cochrane Handbook for Systematic Reviews of Interventions Version 5.1.0 (updated March 2011). The Cochrane Collaboration, 2011. Available from www.handbook.cochrane.org.

\section{Schünemann 2011b}

Schünemann HJ, Oxman AD, Vist GE, Higgins JPT, Deeks JJ, Glasziou P, et al. Chapter 12. Interpreting results and drawing conclusions. In: Higgins JPT, Green S, editor(s). Cochrane Handbook for Systematic Reviews of Interventions Version 5.1.0 (updated March 2011). The Cochrane Collaboration, 2011. Available from www.handbook.cochrane.org.

\section{Segal 2005}

Segal JB, Dzik WH. Paucity of studies to support that abnormal coagulation test results predict bleeding in the setting of invasive procedures: an evidence-based review. Transfusion 2005;45(9):1413-25.

\section{Shander 2007}

Shander A. Financial and clinical outcomes associated with surgical bleeding complications. Surgery 2007;142(4 Suppl):S20-5. 


\section{Shander 2016}

Shander A, Ozawa S, Hofmann A. Activity-based costs of plasma transfusions in medical and surgical inpatients at a US hospital. Vox Sanguinis 2016;111(1):55-61.

\section{Stanworth 2004}

Stanworth SJ, Brunskill SJ, Hyde CJ, McClelland DB, Murphy MF. Is fresh frozen plasma clinically effective? A systematic review of randomized controlled trials. British Journal of Haematology 2004;126(1):139-52.

\section{Stanworth 2007}

Stanworth SJ. The evidence-based use of FFP and cryoprecipitate for abnormalities of coagulation tests and clinical coagulopathy. Hematology, American Society of Hematology Education Program 2007;2007:179-86.

\section{Stanworth 2011a}

Stanworth SJ, Grant-Casey J, Lowe D, Laffan M, New H, Murphy MF, et al. The use of fresh-frozen plasma in England: high levels of inappropriate use in adults and children. Transfusion 2011;51(1):62-70.

\section{Stanworth 2011b}

Stanworth SJ, Walsh TS, Prescott RJ, Lee RJ, Watson DM, Wyncoll D. A national study of plasma use in critical care: clinical indications, dose and effect on prothrombin time. Critical Care 2011;15(2):R108.

\section{Sterne 2011}

Sterne JAC, Egger M, Moher D (editors). Chapter 10. Addressing reporting biases. In: Higgins JPT, Green S, editor(s). Cochrane Handbook for Systematic Reviews of Interventions Version 5.1.0 (updated March 2011). The Cochrane Collaboration, 2011. Available from www.handbook.cochrane.org.

\section{Stokes 2018}

Stokes EA, Wordsworth S, Staves J, Mundy N, Skelly J, Radford K, Stanworth SJ. Accurate costs of blood transfusion: a microcosting of administering blood products in the United Kingdom National Health Service. Transfusion 2018;58(4):846-53.

\section{Subramanian 2018}

Subramanian M. The role of thromboelastography in the emergency medicine, trauma center, and critical care environments. Current Emergency and Hospital Medicine Reports 2018;6:24-30.

\section{Szczepiorkowski 2013}

Szczepiorkowski ZM, Dunbar NM. Transfusion guidelines: when to transfuse. Hematology, American Society of Hematology Education Program 2013;2013:638-44.

\section{Tierney 2007}

Tierney JF, Stewart LA, Ghersi D, Burdett S, Sydes MR. Practical methods for incorporating summary time-to-event data into meta-analysis. Trials 2007;8:16.

\section{Tinmouth 2013}

Tinmouth A, Thompson T, Arnold DM, Callum JL, Gagliardi K, Lauzon $D$, et al. Utilization of frozen plasma in Ontario: a provincewide audit reveals a high rate of inappropriate transfusions. Transfusion 2013;53(10):2222-9.

\section{Toumi 2015}

Toumi M, Urbinati D, EAHC-EU Commission. An EU-wide overview of the market of blood, blood components and plasma derivatives focusing on their availability for patients. Creative Ceutical Report, revised by the Commission to include stakeholders' comments, 2015. Available from ec.europa.eu/health//sites/health/files/blood_tissues_organs/ docs/20150408_cc_report_en.pdf. EU Commission, (accessed 5 February 2017).

\section{Triulzi 2015}

Triulzi D, Gottschall J, Murphy E, Wu Y, Ness P, Kor D, et al. A multicenter study of plasma use in the United States. Transfusion 2015;55(6):1313-9; quiz 1312.

\section{U.S. Government 2016}

U.S. Government. Postmarketing reporting of adverse drug experiences. In: U.S. Government. Code of federal regulations: Title 21 - Food and Drugs. 21 CFR 314.80. U.S. Government Publishing Office, 2016. Available from www.gpo.gov/fdsys/pkg/ CFR-2016-title21-vol5/pdf/CFR-2016-title21-vol5-part314.pdf (accessed 5 February 2017).

\section{van Veen 2011}

van Veen JJ, Spahn DR, Makris M. Routine preoperative coagulation tests: an outdated practice?. British Journal of Anaesthesia 2011;106(1):1-3.

\section{Verghese 2008}

Verghese SG. Elective fresh frozen plasma in the critically ill: what is the evidence?. Critical Care and Resuscitation 2008;10(3):264-8.

\section{Volkow 2005}

Volkow P, Del Rio C. Paid donation and plasma trade: unrecognized forces that drive the AIDS epidemic in developing countries. International Journal of STD \& AIDS 2005;16(1):5-8.

\section{Watson 2009}

Watson GA, Sperry JL, Rosengart MR, Minei JP, Harbrecht BG, Moore EE, et al. Fresh frozen plasma is independently associated with a higher risk of multiple organ failure and acute respiratory distress syndrome. Journal of Trauma Injury Infection and Critical Care 2009;67(2):221-30.

\section{WB 2017}

The World Bank. World bank country and lending groups. datahelpdesk.worldbank.org/knowledgebase/articles/906519world-bank-country-and-lending-groups (accessed 27 December 2017)

\section{Weeder 2014}

Weeder PD, Porte RJ, Lisman T. Hemostasis in liver disease: implications of new concepts for perioperative management.. Transfusion Medicine Reviews 2014;28(3):107-13. 


\section{Whitaker 2016}

Whitaker B, Rajbhandary S, Kleinman S, Harris A, Kamani N. Trends in United States blood collection and transfusion: results from the 2013 AABB Blood Collection, Utilization, and Patient Blood Management Survey. Transfusion 2016;56(9):2173-83.

\section{Whiting 2014}

Whiting D, DiNardo JA. TEG and ROTEM: technology and clinical applications. American Journal of Hematology 2014;89(2):228-32.

\section{WHO 2008}

World Health Organization. Universal Access to Safe Blood Transfusion. Geneva: World Health Organization, 2008.

\section{WHO 2017}

World Health Organization. Global Status Report on Blood Safety and Availability 2016. Geneva: World Health Organization, 2017.

\section{Wong 2007}

Wong MP, Droubatchevskaia N, Chipperfield KM, Wadsworth LD, Ferguson DJ. Guidelines for frozen plasma transfusion. British Columbia Medical Journal 2007;49(6):311-9.

\section{Wu 2001}

Wu Z, Rou K, Detels R. Prevalence of HIV infection among former commercial plasma donors in rural eastern China. Health Policy and Planning 2001;16(1):41-6.

\section{CHARACTERISTICS OFSTUDIES}

Characteristics of included studies [ordered by study ID]

\section{Yaddanapudi 2014}

Yaddanapudi S, Yaddanapudi LN. Indications for blood and blood product transfusion. Indian Journal of Anaesthesia 2014;58(5):538-42.

\section{Yang 2012}

Yang L, Stanworth SJ, Hopewell S, Doree C, Murphy M. Is fresh-frozen plasma clinically effective? An update of a systematic review of randomized controlled trials. Transfusion 2012;52(8):1673-86; quiz 1673.

\section{Zakeri 2017}

Zakeri N, Tsochatzis EA. Bleeding risk with invasive procedures in patients with cirrhosis and coagulopathy. Current Gastroenterology Reports 2017;19(9):45.

\section{References to other published versions of this review} Huber 2017

Huber J, Stanworth SJ, Doree C, Trivella M, Brunskill SJ, Hopewell S, et al. Prophylactic plasma transfusion for patients undergoing non-cardiac surgery. Cochrane Database of Systematic Reviews 2017, Issue 8. [DOI: 10.1002/14651858.CD012745]

* Indicates the major publication for the study

De Pietri 2016

Study design: randomised controlled trial
Study grouping: parallel group
Number of study centres: 1
Power calculation: reduction in blood product transfusion was used as a primary outcome to calcu-
late sample size. Assuming a $20 \%$ difference in the average transfusion requirement (1000 $\mathrm{mL}$ in the
SOC group and $800 \mathrm{~mL}$ in the TEG group) with a $5 \%$ alpha error and a $20 \%$ beta error, 30 patients in
each group were required

Length of follow-up: 90 days from procedure

Study duration for participants: 90 days

Stopping rules: not reported

Baseline characteristics
Thromboelastography threshold
- Age: $57.8 \pm 9.4$
- Sex: male $16(53.3 \%)$
- Type of surgery: paracentesis $(n=12)$; central vein cannulation $(n=1)$; variceal band ligation $(n=6) ;$
hepatic resection $(n=3)$; abdominal surgery $(n=2)$; endoscopic polypectomy $(n=3)$; radiofrequency
ablation $(n=2) ;$ thoracotomy $(n=1)$

Prophylactic plasma transfusion for patients without inherited bleeding disorders or anticoagulant use undergoing non-cardiac surgery 35 or invasive procedures (Review)

Copyright @ 2019 The Cochrane Collaboration. Published by John Wiley \& Sons, Ltd. 
- $P T: 44.3 \pm 16.6(\%)$

- INR: $1.87 \pm 0.55$

- $a P T T: 1.35 \pm 0.26$

- TEG/ROTEM: $\mathrm{R}$ (minutes) $19.4 \pm 11 \mathrm{~K}$, (minutes) $11.8 \pm 60 \mathrm{MA}$, (mm) $21.5 \pm 12.6$, $\alpha$-angle $35.5 \pm 9.30 \mathrm{CL} 30$, (\%) $96.9 \pm 12.2 \mathrm{LY} 30,(\%) 0.15 \pm 0.37$

- $H b: 109 \pm 19$

- Platelet count: $56.5 \pm 32.5$

- Number with a coagulopathy: 12

- Underlying diagnosis: liver cirrhosis

- Ethnic group: not reported

Standard of care (laboratory parameters)

- Age: $58.6 \pm 12.1$

- Sex: male 22 (73.3\%)

- Type of surgery: paracentesis $(n=7)$; thoracocentesis $(n=5)$; central vein cannulation $(n=2)$; transjugular intrahepatic portosystemic shunt procedure $(n=1)$; variceal band ligation $(n=4)$; hepatic resection ( $n=2)$; abdominal surgery $(n=2)$; radiofrequency ablation $(n=1)$; liver biopsy $(n=3)$; biopsy of sites other than liver $(n=1)$; abdominal drainage $(n=1)$; endoscopic retrograde cholangiopancreatography $(E R C P)$ with sphincterotomy $(n=1)$

- $P T: 40.4 \pm 14.3(5)$

- INR: $2.01 \pm 0.69$

- $a P T T: 1.34 \pm 0.20$

- TEG/ROTEM: R (minutes) $18.7 \pm 12.8 \mathrm{~K}$, (minutes) $10.9 \pm 7.90 \mathrm{MA}$, $(\mathrm{mm}) 25.0 \pm 16.8$, $\alpha$-angle $36.7 \pm 10.8$ CL30, (\%) $99.3 \pm 1.20$ LY30, (\%) $0.12 \pm 0.37$

- $H b: 103 \pm 13$

- Platelet count: $61.3 \pm 41.9$

- Number with a coagulopathy: 16

- Underlying diagnosis: liver cirrhosis

- Ethnic group: not reported

Included criteria: cirrhosis (histologically or imaging proven); age 18 to 80 years; undergoing an invasive procedure; INR $>1.8$, and/or platelet count $50 \times 10^{9} / \mathrm{L}$

Excluded criteria: ongoing bleeding; previous or current thrombotic events defined as any documented blood clot in a venous or arterial vessel; anti-platelet or anticoagulant therapy at the time of enrolment or that had been discontinued less than 7 days before evaluation for the study; presence of documented infection or sepsis according to ACCP-SCCM criteria; haemodialysis in the previous 7 days

Pretreatment: no statistical imbalance between arms in baseline characteristics. More men than women in standard of care group

Number screened: 153

Number recruited: 60

Type of surgery: 28 participants underwent procedures with risk of bleeding lower than $3 \%$ according to the literature: paracentesis $(n=19)$; thoracocentesis $(n=5)$; central vein cannulation $(n=3)$; transjugular intrahepatic portosystemic shunt procedure $(n=1)$. 32 participants underwent procedures with bleeding risk exceeding $3 \%$ : variceal band ligation $(n=10)$; hepatic resection $(n=5)$; abdominal surgery $(n=4)$; endoscopic polypectomy $(n=3)$; radiofrequency ablation $(n=3)$; liver biopsy $(n=$ $3)$; biopsy of sites other than liver $(n=1)$; abdominal drainage $(n=1)$; endoscopic retrograde cholangiopancreatography (ERCP) with sphincterotomy $(n=1)$; thoracotomy $(n=1)$

\section{Number excluded: 93}

Thromboelastography threshold 
De Pietri 2016 (Continued)

\section{- Type of plasma: FFP}

- Dose of plasma: $10 \mathrm{~mL} / \mathrm{kg}$ ideal body weight

- Timing of transfusion: before procedure, if R-time $>40$ minutes

Standard of care (laboratory parameters)

- Type of plasma: FFP

- Dose of plasma: $10 \mathrm{~mL} / \mathrm{kg}$ ideal body weight

- Timing of transfusion: before procedure, if INR $>1.8$

All-cause mortality
Major bleeding
Number of individuals requiring a transfusion
Serious adverse events due to plasma transfusion
Serious adverse events due to surgery
Return to theatre to treat bleeding

Identification

Sponsorship source: University of Modena and Reggio Emilia

Country: Italy

Setting: liver patients

Comments:

Author's name: Lesley De Pietri

Institution: Azienda Ospedaliero Universitaria Policlinico di Modena

Email:

Address: Division of Anesthesiology and Intensive Care Unit, Department of Surgery, Azienda Ospedaliero Universitaria Policlinico di Modena, Modena, Italy

Trial Registration: NCT02362178

Date trial registered: 23 January 2015

Date trial started recruiting participants: February 2011

Date trial completed recruitment: August 2014

Conflicts of interest of study authors: not reported

\section{Notes}

\section{Risk of bias}

\begin{tabular}{lll}
\hline Bias & Authors' judgement & Support for judgement \\
\hline $\begin{array}{l}\text { Random sequence genera- } \\
\text { tion (selection bias) }\end{array}$ & Low risk & $\begin{array}{l}\text { Quote: "randomization was performed electronically by block of } 4 \text { in a 1:1 } \\
\text { rate" }\end{array}$ \\
\hline $\begin{array}{l}\text { Allocation concealment } \\
\text { (selection bias) }\end{array}$ & Unclear risk & Comment: not reported \\
\hline
\end{tabular}

Prophylactic plasma transfusion for patients without inherited bleeding disorders or anticoagulant use undergoing non-cardiac surgery 
De Pietri 2016 (Continued)

Blinding of participants and personnel (performance bias)

All outcomes
High risk Quote: "study design: in this randomised, controlled, open-label, intention-totreat (ITT) trial..."

Comment: apart from all-cause mortality, the study is at risk of bias

\section{Blinding of outcome as- High risk} sessment (detection bias) All outcomes
Quote: "in this randomised, controlled, open-label...trial"

Quote: "no transfusion side effects were reported in the TEG group, whereas an allergic reaction during FFP infusion occurred in the SOC group"

Comment: blinding of assessors is not described. Potential risk of detection bias in all outcomes apart from all-cause mortality is due to knowledge of allocated interventions by outcome assessors

Incomplete outcome data Low risk Comment: all randomised participants included in ITT analysis
(attrition bias)

All outcomes

Selective reporting (re- High risk
porting bias)
Comment: study protocol described. Time frame for reporting of bleeding episodes "48 hours from admission". Time span for reporting the primary outcome is not clear from the report's description

Comment: protocol described transfusion-related side effect time scale 48 hours. The report reduced this time scale to 6 hours

Comment: reporting of blood test abnormalities post procedure was not a described outcome in the protocol nor in the report on methods/efficacy assessment. Aside from $\mathrm{Hb}$, INR, and PLT count, other variables were not shown (quote: "none of the other variables showed statistical difference..."), precluding potential for meta-analysis

Comment: comparison of blood product cost between groups was a secondary outcome described in the protocol. This was not described in the report on efficacy assessment nor in the results

Other bias Low risk

Baseline characteristics

Quote: "no significant differences in terms of age, sex, clinical features, cirrhosis prognostic scores, and clotting parameters were present between the two study groups at baseline"

Comment: a table describing demographic, clinical, and biochemical characteristics of patients enrolled, together with $P$ values, is concordant with the above quote

Study design: randomised controlled trial
Study grouping: parallel group
Power calculation: not reported
Stopping rules: not reported
Length of follow-up: 12 months

Study duration for participants: not reported 


\section{Number of study centres: 1}

Participants

\section{Baseline characteristics}

Prophylactic plasma transfusion before surgery

- Age: not reported

- Sex: not reported

- Type of surgery: needle biopsy

- PT: not reported

- INR: not reported

- aPTT: not reported

- TEG/ROTEM: not reported

- Hb: not reported

- Platelet count: not reported

- Number with a coagulopathy: 10

- Underlying diagnosis: chronic liver disease

- Ethnic group: not reported

Alternative haemostatic agents

- Age: not reported

- Sex: not reported

- Type of surgery: needle biopsy

- $P T$ : not reported

- INR: not reported

- aPTT: not reported

- TEG/ROTEM: not reported

- $H b$ : not reported

- Platelet count: not reported

- Number with a coagulopathy: 11

- Underlying diagnosis: chronic liver disease

- Ethnic group: not reported

Included criteria: chronic liver disease; undergoing needle biopsy; prothrombin time, kaolin-activated partial thromboplastin time, and 'Normotest' results all above the upper normal limit

Excluded criteria: bleeding time 7 minutes or longer

Pretreatment: not reported

Number screened: 30

Number recruited: 21

Number excluded: 9

Type of surgery: needle biopsy $(n=30)$

\section{Intervention characteristics}

Prophylactic plasma transfusion before surgery

- Type of plasma/alternative agent: FFP

- Dose of plasma/alternative agent: $12 \mathrm{~mL} / \mathrm{kg}$

- Timing of transfusion: before biopsy, over 45 to 60 minutes

Alternative haemostatic agents 
Mannucci 1976 (Continued)

- Type of plasma/alternative agent: Prothromplex (Immuno, Vienna) containing factors II, IX, and X, followed by factor VII concentrate (Immuno, Vienna)

- Dose of plasma/alternative agent: Prothromplex 25 units/kg; factor VII concentrate 25 units/kg

- Timing of transfusion: before biopsy, over 15 minutes

\begin{tabular}{ll}
\hline Outcomes & Major bleeding \\
& Serious adverse events due to plasma transfusion \\
& Venous and arterial thromboembolism \\
Coagulation test abnormalities
\end{tabular}

Identification

Sponsorship source: not reported

Country: Italy

Setting: liver patients

Comments:

Author's name: P.M. Mannucci

Institution: University of Milan and Haemophilia and Thrombosis Centre

Email: not reported

Address: 3rd Department of Clinical Medicine, University of Milan and Haemophilia and Thrombosis Centre, Angelo Bianchi Bonomi, Via Pace 15, 20122 Milan, Italy

Trial registration: not reported

Date trial registered: not reported

Date trial completed recruitment: not reported

Date trial started recruiting participants: not reported

Conflicts of interest of study authors: not reported

\section{Notes}

\section{Risk of bias}

\begin{tabular}{|c|c|c|}
\hline Bias & Authors' judgement & Support for judgement \\
\hline \multirow[t]{2}{*}{$\begin{array}{l}\text { Random sequence genera- } \\
\text { tion (selection bias) }\end{array}$} & Unclear risk & $\begin{array}{l}\text { Quote: "21 consecutive patients were randomly allocated by a system of } \\
\text { sealed envelopes to treatment with either FFP or concentrates" }\end{array}$ \\
\hline & & Comment: system of random sequence generation unclear \\
\hline \multirow[t]{2}{*}{$\begin{array}{l}\text { Allocation concealment } \\
\text { (selection bias) }\end{array}$} & Unclear risk & $\begin{array}{l}\text { Quote: "21 consecutive patients were randomly allocated by a system of } \\
\text { sealed envelopes to treatment with either FFP...or concentrates" }\end{array}$ \\
\hline & & $\begin{array}{l}\text { Comment: it is unclear from the report whether the envelopes were opaque } \\
\text { and sequentially numbered to reduce risk of allocation bias }\end{array}$ \\
\hline $\begin{array}{l}\text { Blinding of participants } \\
\text { and personnel (perfor- } \\
\text { mance bias) } \\
\text { All outcomes }\end{array}$ & Unclear risk & Comment: not reported whether participants and personnel were blinded \\
\hline
\end{tabular}

Prophylactic plasma transfusion for patients without inherited bleeding disorders or anticoagulant use undergoing non-cardiac surgery 
Mannucci 1976 (Continued)

Blinding of outcome as- Unclear risk Comment: not reported whether outcome assessors were blinded to the intersessment (detection bias) vention

All outcomes

\begin{tabular}{lll}
$\begin{array}{l}\text { Incomplete outcome data } \\
\text { (attrition bias) } \\
\text { All outcomes }\end{array}$ & Unclear risk & $\begin{array}{l}\text { Comment: attrition rate/number analysed/number lost to follow-up is not re- } \\
\text { ported. Therefore information is insufficient to permit a judgement }\end{array}$ \\
\hline $\begin{array}{l}\text { Selective reporting (re- } \\
\text { porting bias) }\end{array}$ & Unclear risk & $\begin{array}{l}\text { Comment: no protocol or trial registration to assess whether all planned out- } \\
\text { comes were reported }\end{array}$
\end{tabular}

Other bias Unclear risk Comment: baseline characteristics were not reported

Müller 2015

Study design: randomised controlled trial
Study grouping: parallel group
Power calculation: based on the assumption that the occurrence of major bleeding in patients with a
coagulopathy undergoing invasive procedures was less than $1 \%$. Group size calculation was focused on
demonstrating non-inferiority. With a sample size in each group of 198, a 1-sided Z test with continuity
correction (pooled) achieved $80 \%$ power to reject the null hypothesis that the proportion of bleeding
patients in the experimental group (no FFP transfusion) was higher than, that is, inferior to, the propor-
tion in the control group (FFP transfusion) with a margin of 0.03. It was assumed that the expected dif-
ference in proportions is zero and the proportion in the control group is 0.01 . The 1-sided significance
level of the test was targeted at 0.05 . Therefore, study authors intended to enrol 200 patients per treat-
ment arm, 400 in total. Owing to slow inclusion, the trial was stopped before the pre-defined target en-
rolment was reached

\section{Stopping rules: not reported}

Length of follow-up: 28 days (according to clinicaltrials.gov database and protocol. Not described in report)

\section{Study duration for participants: 28 days}

Number of study centres: 4

Participants

\section{Baseline characteristics}

Prophylactic plasma transfusion before surgery

- Age: median 64 years (IQR 54 to 70 )

- Sex: male 26; female 12

- Type of surgery: central venous catheter $(n=29)$; chest tube $(n=4)$; tracheotomy $(n=2)$; abdominal drain $(\mathrm{n}=3)$

- PT: not reported

- INR: median 1.8 (IQR 1.5 to 2.2 )

- aPTT: median 43 (IQR 38 to 52)

- TEG/ROTEM: not reported

- Hb: median 93 (IQR 84 to 102 )

- Platelet count: median $92 \times 10^{9} / \mathrm{L}$ (IQR 52 to 180$)$

- Number with a coagulopathy: DIC 17

- Underlying characteristic: pulmonary disease $(n=5)$; liver disease $(n=6)$; cardiac failure $(n=6)$; mechanical ventilation within 24 hours $(n=32)$; sepsis within 24 hours $(n=18)$; pneumonia within 24 hours $(n=10)$ 
- Ethnic group: not reported

No prophylactic plasma transfusion before surgery (colloid, crystalloid, placebo, or no treatment)

- Age: median 66 years (IQR 62 to 72 )

- Sex: male 18; female 20

- Type of surgery: central venous catheter $(n=29)$; chest tube $(n=3)$; tracheotomy $(n=2)$; abdominal drain $(n=4)$

- PT: not reported

- INR: median 1.9 (IQR 1.6 to 2.2)

- aPTT: median 41 (IQR 36 to 49)

- TEG/ROTEM: not reported

- $H b$ : median 93 (IQR 86 to 104)

- Platelet count: median $110 \times 10^{9} / \mathrm{L}$ (IQR 52 to 183 )

- Number with a coagulopathy: DIC 14

- Underlying characteristic: pulmonary disease $(n=4)$; liver disease $(n=17)$; cardiac failure $(n=6)$; mechanical ventilation within 24 hours $(n=29)$; sepsis within 24 hours $(n=18)$; pneumonia within 24 hours $(n=9)$

- Ethnic group: not reported

Included criteria: adults in intensive care (aged 18 years and older) with INR $\geq 1.5$ undergoing insertion of a central venous catheter, thoracocentesis, percutaneous tracheotomy, or drainage of abscess or fluid collection $(n=81)$

Excluded criteria: people with clinically overt bleeding (defined as decrease in haemoglobin $(\mathrm{Hb})>$ $160 \mathrm{~g} / \mathrm{L}$ or need for transfusion or haemodynamic instability due to bleeding at the time of the procedure); thrombocytopenia $<30 \times 10^{9} / \mathrm{L}$; patients treated with vitamin $\mathrm{K}$ antagonists, activated protein $\mathrm{C}$, abciximab, tirofiban, ticlopidine, or prothrombin complex concentrates; patients with a history of congenital or acquired coagulation factor deficiency or bleeding diathesis; use of heparin $<1$ hour before the procedure; use of therapeutic doses of low molecular weight heparin $<12$ hours before the procedure

Pretreatment: more men than women in the FFP transfusion group $(P=0.09)$. Greater number of patients with liver disease in the 'no FFP transfusion' group, reaching statistical significance $(P=0.006)$

Number screened: a total of 1478 patients had an INR $\geq 1.5$ and $\leq 3.0$

Number recruited: 81 participants. Five did not undergo an intervention and were therefore excluded from further analysis (2 FFP; 3 no FFP)

Number excluded: 615 patients did not fulfil inclusion criteria, leaving 263 patients with an INR $\geq 1.5$ and $\leq 3.0$ scheduled to undergo a pre-defined intervention. Of these, 65 patients declined informed consent. An additional 83 patients were missed and 34 patients did not participate for other reasons, including refusal of treating physicians to include a specific patient $(3.8 \%)$

Type of surgery: central venous catheter insertion $(n=58)$; chest tube insertion $(n=7)$; tracheotomy ( $n$ $=4)$; abdominal drain insertion $(n=7)$

\section{Intervention characteristics}

Prophylactic plasma transfusion before surgery

- Type of plasma: FFP (manufactured by Sanquin, the Dutch National Bloodbank)

- Dose of plasma: $12 \mathrm{~mL} / \mathrm{kg}$

- Timing of transfusion: before invasive procedure

No prophylactic plasma transfusion before surgery (no treatment)

\begin{tabular}{ll}
\hline Outcomes & All-cause mortality \\
& Major bleeding
\end{tabular}

Prophylactic plasma transfusion for patients without inherited bleeding disorders or anticoagulant use undergoing non-cardiac surgery 
Müller 2015 (Continued)

Number of transfusions per participant

Serious adverse events due to plasma transfusion

ITU length of stay

Return to theatre to treat bleeding

Coagulation test abnormalities

Identification

Sponsorship source: Academisch Medisch Centrum - Universiteit van Amsterdam (AMC-UvA)

Country: Netherlands

Setting: patients in intensive care units undergoing invasive procedures

Comments:

Author's name: Marcella C.A. Müller

Institution: Department of Intensive Care Medicine, Academic Medical Center, University of Amsterdam

Email: m.c.muller@amc.uva.nl.

Address: Department of Intensive Care Medicine, Academic Medical Center, University of Amsterdam, Meibergdreef 9, 1105 AZ Amsterdam, the Netherlands

Trial registration: Dutch trial registry NTR2262NCT01143909

Date trial registered: 26 March 2010

Date trial completed recruitment: June 2013

Date trial started recruiting participants: May 2010

Conflicts of interest of study authors: NPJ reports grants from Netherlands Organisation for Scientific Research (NWO) during the conduct of the study. The other study authors disclose no conflicts of interest

\section{Notes}

\section{Risk of bias}

Bias Authors' judgement Support for judgement

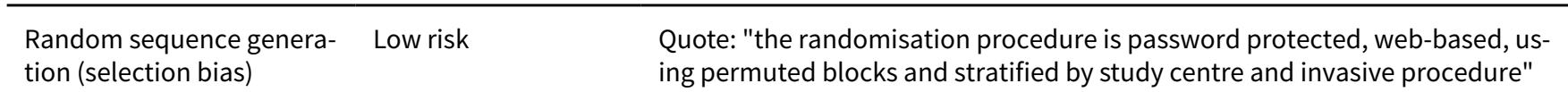

\begin{tabular}{lll}
\hline $\begin{array}{l}\text { Allocation concealment } \\
\text { (selection bias) }\end{array}$ & Low risk & $\begin{array}{l}\text { Quote: "the randomisation procedure is password protected, web-based, us- } \\
\text { ing permuted blocks and stratified by study centre and invasive procedure" }\end{array}$ \\
\hline $\begin{array}{l}\text { Blinding of participants } \\
\text { and personnel (perfor- } \\
\text { mance bias) }\end{array}$ & High risk & $\begin{array}{l}\text { Quote: "since manufacturing a completely matched placebo in full compliance } \\
\text { with the current good manufacturing practice standards was considered not } \\
\text { possible, a prospective, randomised, open-label, blinded endpoint evaluation } \\
\text { (PROBE) design was chosen" }\end{array}$ \\
& $\begin{array}{l}\text { Comment: investigators and clinicians were unblinded to the intervention. It is } \\
\text { unclear how many participants were sedated and were not aware of the inter- } \\
\text { vention }\end{array}$ \\
\hline
\end{tabular}

Blinding of outcome as- Low risk sessment (detection bias)
Quote: "the potential bleeding site was assessed by a physician blinded to the intervention who filled out a predefined bleeding score form consisting

Prophylactic plasma transfusion for patients without inherited bleeding disorders or anticoagulant use undergoing non-cardiac surgery 
Müller 2015 (Continued)

All outcomes of blood pressure, heart rate, $\mathrm{Hb}$ level, and occurrence of procedure-related bleeding with or without the need for intervention or transfusion. Subsequently this blinded physician assigned a score of major bleeding, minor bleeding, or no bleeding at 1 and 24 hours after the intervention"

\begin{tabular}{|c|c|c|}
\hline $\begin{array}{l}\text { Incomplete outcome data } \\
\text { (attrition bias) }\end{array}$ & Low risk & $\begin{array}{l}\text { Comment: all participants who were randomised and underwent an invasive } \\
\text { procedure were included in the analysis }\end{array}$ \\
\hline
\end{tabular}

Comment: all participants who were randomised and underwent an invasive

\section{Selective reporting (re- High risk} porting bias) Quote (from protocol): regarding primary outcomes: "relevant bleeding is de-
fined using a validated tool (HEME) in the critically ill"

Quote (from report): "the HEME tool used to assess procedure-related relevant bleeding in our trial has been validated for ICU patients, with a high interrater agreement"

Comment: primary outcome was reported using prescribed measurement method

Quote (from report): "... the World Health Organization bleeding scale...is validated for patients with cancer but not in the critically ill. A possible disadvantage of the HEME tool is that some items, such as decrease in systolic blood pressure or increase in heart rate, may also occur in the absence of bleeding. However, assessors were asked to consider such physiologic changes only if they occurred in the absence of other causes"

Comment: limitations and method of primary outcome assessment described. Risk of under-reporting in the presence of concomitant pathologies (e.g. sepsis, cardiogenic shock, hepato-renal syndrome), causing physiological dysfunction

Quote (from protocol): "the occurrence of relevant bleedings (using a binary variable), expressed in a relative risk estimate and absolute risk increase"

Quote (from report): "the primary endpoint of procedure-related major bleeding only occurred once, rendering planned non-inferiority analysis impossible. Therefore, a post hoc non-inferiority analysis for all types of bleeding complications (major and minor) was performed according to the method of Dunnett and Gent"

Quote (from report): "post hoc analysis showed a trend toward non-inferiority with a $5 \%$ higher bleeding rate in the transfused group compared to non-transfused patients"

Comment: post hoc analysis (due to low incidence of primary outcome measure on a background of patient under-recruitment) was used, which was not pre-defined in the protocol. Adverse event outcome measure (predefined in protocol) was not reported. Therefore potential risk of reporting bias was due to selective outcome reporting
High risk
Comment: the major limitation of this trial is that it was stopped early due to slow inclusion. Despite the addition of extra recruitment sites, the study was able to randomise only $20 \%$ of the targeted participant number. There was an imbalance in the number of participants with a history of liver disease between treatment arms. 45\% (17/38) of participants had liver disease in the no FFP arm, whereas only 16\% (6/38) had liver disease in the FFP arm

Methods Study design: randomised trial

Prophylactic plasma transfusion for patients without inherited bleeding disorders or anticoagulant use undergoing non-cardiac surgery or invasive procedures (Review)

Copyright @ 2019 The Cochrane Collaboration. Published by John Wiley \& Sons, Ltd. 
NCT00953901 (Continued)

\section{Study grouping: parallel group}

Masking: double-blinded

Included criteria:
Participants $\quad$ Consenting hospitalised adult
- F 18 years old
- Patients with abnormal INR (INR 1.6 to 3 ) due to vitamin K depletion or antagonism (Coumadin and/
or broad-spectrum antibiotics) or liver insufficiency
- Patients who are about to undergo 1 of the 3 common minimally invasive procedures (thoracocente-
sis, abdominal paracentesis, and central vein cannulation)
Excluded criteria: not stated
Drug: fresh frozen plasma transfusion
Primary outcome measures:
New-onset pulmonary edema
Secondary outcome measures:
Postprocedural bleeding complications
Number of blood product transfusions
Hospital mortality
Length of intensive care unit and hospital stay

Identification

Sponsorship source: Mayo Clinic

Country: United States of America

Setting: patients undergoing commonly performed invasive procedures

Institution: Mayo Clinic, Rochester.

Address: Mayo Clinic, Rochester, Minnesota, United States, 55905

Trial registration: NCT00953901

Date trial registered: 6 August 2009

Date trial started recruiting participants: July 2006

Date trial completed recruitment: December 2007

\begin{tabular}{lll}
\hline Notes & $\begin{array}{l}\text { Study completion: December } 2008 \\
\text { Recruitment: } \mathrm{N}=2\end{array}$ \\
\hline Risk of bias & Authors' judgement & Support for judgement \\
\hline Bias & Unclear risk & $\begin{array}{l}\text { Comment: trial recruitment completed in } 2008 \text { with } 2 \text { participants. Given no } \\
\text { trial report was published, and based on available information in the trial reg- } \\
\text { istry, available information is insufficient to permit a judgement }\end{array}$ \\
\hline $\begin{array}{l}\text { Random sequence genera- } \\
\text { tion (selection bias) }\end{array}$ &
\end{tabular}

Prophylactic plasma transfusion for patients without inherited bleeding disorders or anticoagulant use undergoing non-cardiac surgery 


\section{NCT00953901 (Continued)}

Allocation concealment (selection bias)

Unclear risk

Comment: trial recruitment completed in 2008 with 2 participants. Given no trial report was published, and based on available information in the trial registry, available information is insufficient to permit a judgement

\begin{tabular}{|c|c|c|}
\hline $\begin{array}{l}\text { Blinding of participants } \\
\text { and personnel (perfor- } \\
\text { mance bias) }\end{array}$ & Unclear risk & $\begin{array}{l}\text { Comment: trial recruitment completed in } 2008 \text { with } 2 \text { participants. Given no } \\
\text { trial report was published, and based on available information in the trial reg- } \\
\text { istry, available information is insufficient to permit a judgment }\end{array}$ \\
\hline
\end{tabular}

Blinding of outcome as- Unclear risk sessment (detection bias) All outcomes
Comment: trial recruitment completed in 2008 with 2 participants. Given no trial report was published, and based on available information in the trial registry, available information is insufficient to permit a judgement

\begin{tabular}{|c|c|c|}
\hline $\begin{array}{l}\text { Incomplete outcome data } \\
\text { (attrition bias) } \\
\text { All outcomes }\end{array}$ & Unclear risk & $\begin{array}{l}\text { Comment: trial recruitment completed in } 2008 \text { with } 2 \text { participants. Given no } \\
\text { trial report was published, and based on available information in the trial reg } \\
\text { istry, available information is insufficient to permit a judgement }\end{array}$ \\
\hline
\end{tabular}

\begin{tabular}{lll}
\hline $\begin{array}{l}\text { Selective reporting (re- } \\
\text { porting bias) }\end{array}$ & Unclear risk & $\begin{array}{l}\text { Comment: trial recruitment completed in 2008 with 2 participants. Given no } \\
\text { trial report was published, and based on available information in the trial reg- } \\
\text { istry, available information is insufficient to permit a judgement }\end{array}$ \\
\hline Other bias & Unclear risk & $\begin{array}{l}\text { Comment: trial recruitment completed in 2008 with 2 participants. No trial re- } \\
\text { port was published on assessment of other bias such as differences in baseline } \\
\text { characteristics }\end{array}$ \\
\hline
\end{tabular}

Study design: randomised controlled trial
Study grouping: parallel group
Power calculation: reduction in bleeding was used as a primary outcome to calculate sample size. As-
suming a $15 \%$ absolute decrease in bleeding following correction of subclinical bleeding disorders with
a $5 \%$ alpha error and a $20 \%$ beta error, 76 patients in each group were required

Stopping rules: not reported

Length of follow-up: unclear; outcomes up to 12 hours post procedure

Study duration for participants: unclear; outcomes at up to 12 hours post procedure

Number of study centres: 1

\section{Participants Baseline characteristics}

Prophylactic plasma or platelet transfusion or both before percutaneous dilatational tracheotomy

- Age: median (IQR) 64 (56 to 72 )

- Sex (male/female): 22/13

- Type of surgery: percutaneous dilatational tracheotomy

- PT: mean (SD) 16.0 (1.2)

- INR: not reported

- aPTT: not reported

- TEG/ROTEM: not reported

- $H b$ : not reported

- Platelet count: median (IQR) 81 (63 to 85)

- Number with a coagulopathy: 19/35

Prophylactic plasma transfusion for patients without inherited bleeding disorders or anticoagulant use undergoing non-cardiac surgery 
- Underlying diagnosis: post surgery $(n=5)$; coma $(n=3)$; cardiac arrest $(n=4)$; acute respiratory failure $(n=19)$; trauma $(n=2)$; other reason for mechanical ventilation $(n=2)$; complicated weaning $(n=10)$; expected prolonged duration of mechanical ventilation $(n=12)$; need for frequent airway suctioning $(n=3)$; low GCS $(n=5)$; critical illness polyneuromyopathy $(n=4)$; other reason for tracheotomy $(n=$ $1)$; acute renal failure $(n=9)$; chronic renal failure $(n=2)$; sepsis $(n=7)$; massive transfusion $(n=5)$; haematological malignancy $(\mathrm{n}=2)$

- Ethnic group: not reported

No prophylactic plasma or platelet transfusion or both before percutaneous dilatational tracheotomy

- Age: median (IQR) 68 (60 to 76 )

- Sex (male/female): $16 / 21$

- Type of surgery: percutaneous dilatational tracheotomy

- PT: mean (SD) 16.6 (1.1)

- INR: not reported

- aPTT: not reported

- TEG/ROTEM: not reported

- $H b$ : not reported

- Platelet count: median (IQR) 56 (47 to 70)

- Number with a coagulopathy: 22/37

- Underlying diagnosis: post surgery $(n=9)$; coma $(n=4)$; cardiac arrest $(n=4)$; acute respiratory failure $(n=18)$; trauma $(n=1)$; other reason for mechanical ventilation $(n=1)$; complicated weaning $(n=17)$; expected prolonged duration of mechanical ventilation $(n=4)$; need for frequent airway suctioning $(n=2)$; low GCS $(n=6)$; critical illness polyneuromyopathy $(n=5)$; other reason for tracheotomy $(n=$ $3)$; acute renal failure $(n=3)$; chronic renal failure $(n=2)$; sepsis $(n=9)$; massive transfusion $(n=5)$; haematological malignancy $(n=3)$

- Ethnic group: NR

Included criteria: patients planned for bedside PDT with mild coagulation disorders (prothrombin time (PT) 14.7 to 20.0 seconds and/or platelet count 40 to $100 \times 10^{9} / \mathrm{L}$ ) and/or active treatment with acetylsalicylic acid at any dose were eligible

Excluded criteria: age < 18 years; need for surgical tracheotomy; contraindications to transfusion of blood products; use of clopidogrel. A patient would also be excluded from participation in this trial if the attending physician insisted on the need for transfusion of FFP and/or platelets

Pretreatment: no difference with regard to gender, age (years), APACHE II score. Time from admission to tracheotomy (days), reason for mechanical ventilation, post-surgical, coma, cardiac arrest, acute respiratory failure, trauma, reason for tracheotomy, complicated weaning, need for frequent airway suctioning, low GCS score; possible reasons for coagulation disorders, acute renal failure, chronic renal failure, sepsis, massive transfusion, liver cirrhosis, haematological, duration of mechanical ventilation, length of stay in ICU, ICU mortality, hospital mortality. Two significant differences were expected prolonged duration of mechanical ventilation AND platelet count more in men than in women (not statistically significant)

\section{Number screened: 355}

Number recruited: randomised 72; 4 in each arm did not undergo PDT*

Number excluded: 283 (refused consent 27, surgical procedure 53, clopidogrel 13, PT > 20 seconds 11, normal coagulation 179)

Type of surgery: percutaneous dilatational tracheotomy

Prophylactic plasma or platelet transfusion or both before percutaneous dilatational tracheotomy

- Type of plasma: FFP

- Dose of plasma: participants with prolonged PT (normal values are between 11.0 and 14.7 seconds) assigned to the "correction group" received 1 or 2 units of FFP ( 1 unit contains $300 \mathrm{~mL}$ of FFP: if the

Prophylactic plasma transfusion for patients without inherited bleeding disorders or anticoagulant use undergoing non-cardiac surgery or invasive procedures (Review)

Copyright (c) 2019 The Cochrane Collaboration. Published by John Wiley \& Sons, Ltd. 
PT was between 14.7 and 18.0 seconds, the patient received 1 unit of FFP; if the PT was between 18.0 and 20.0 seconds, the patient received 2 units of FFP)

- Type of platelets: platelet concentrates prepared from 5 pooled buffy coats

- Dose of platelets: participants with a low platelet count and/or active use of acetylsalicylic acid received 5 units of platelet concentrates

- Timing of transfusion: before procedure

No prophylactic plasma or platelet transfusion or both before percutaneous dilatational tracheotomy

- Type of plasma/platelets: NA

- Dose of plasma/platelets: participants assigned to the "no correction" group received neither plasma nor platelets. However, FFP and/or platelets were made available for immediate transfusion in case bleeding occurred during or after PDT

- Timing of transfusion: NA

\begin{tabular}{ll}
\hline Outcomes & All-cause mortality \\
& Major bleeding \\
Number of individuals requiring a transfusion \\
Use of haemostatic agents \\
Volume of blood loss per participant \\
ITU length of stay
\end{tabular}

Identification

Sponsorship source: Academic Medical Centre (AMC) (The Netherlands) - Department of Intensive Care

Country: The Netherlands

Setting: University hospital in The Netherlands/1 centre ICU

Comments:

Author's name: Denise P. Veelo

Institution: Department of Intensive Care Medicine and Department of Anaesthesiology, Academic Medical Centre, University of Amsterdam

Email: d.p.veelo@amc.uva.nl

Address: Department of Intensive Care Medicine, Academic Medical Centre, University of Amsterdam, Meibergdreef 91105 AZ Amsterdam, The Netherlands

Trial registration: NTR694 ISRCTN31808827

Date trial registered: NTR: 29 May 2006; ISRCTN: 19 July 2006

Date trial completed recruitment: NTR/ISRCTN: 01 July 2009; Study report: October 2009

Date trial started recruiting participants: 1 July 2006

Conflicts of interest of study authors: study authors declare no conflicts of interest

Notes 
Veelo 2012 (Continued)

Risk of bias

\begin{tabular}{lll}
\hline Bias & Authors' judgement & Support for judgement \\
\hline $\begin{array}{l}\text { Random sequence genera- } \\
\text { tion (selection bias) }\end{array}$ & Low risk & $\begin{array}{l}\text { Quote: "a computer-generated randomisation scheme was used. Each assign- } \\
\text { ment ("correction" or "no correction") was recorded on a piece of paper fold- } \\
\text { ed three times and enclosed in a consecutively numbered, opaque, sealed en- } \\
\text { velope" }\end{array}$ \\
\hline $\begin{array}{l}\text { Allocation concealment } \\
\text { (selection bias) }\end{array}$ & Low risk & $\begin{array}{l}\text { Quote: "each assignment ("correction" or "no correction") was recorded on a } \\
\text { piece of paper folded three times and enclosed in a consecutively numbered, } \\
\text { opaque, sealed envelope" }\end{array}$ \\
& Comment: appropriate concealment
\end{tabular}

Blinding of participants

High risk and personnel (perfor-

Comment: open-label design study. At high risk of bias apart from all-cause mance bias)

All outcomes mortality

\begin{tabular}{lll}
\hline Blinding of outcome as- & High risk & $\begin{array}{l}\text { Comment: open-label design study. Outcomes at high risk of bias apart from } \\
\text { sessment (detection bias) }\end{array}$
\end{tabular}

All outcomes

\begin{tabular}{|c|c|c|}
\hline $\begin{array}{l}\text { Incomplete outcome data } \\
\text { (attrition bias) } \\
\text { All outcomes }\end{array}$ & Low risk & $\begin{array}{l}\text { Comment: clear CONSORT flow diagram. Equal numbers of patients not under- } \\
\text { going PDT }(N=4) \text { after randomisation present in each study arm. Attrition bias } \\
\text { likely to be low }\end{array}$ \\
\hline
\end{tabular}

Selective reporting (re- High risk porting bias)

\author{
Comment: no protocol available, but registered ISRCTN31808827 \\ Primary outcome measures: \\ Volume of blood loss during PDT \\ Intensity of intra-tracheal bleeding \\ Time until no blood is visible in tracheal aspirates \\ These were all reported
}

Secondary outcome measures:

Number of blood products used during and after tracheotomy - not clearly reported

Other bias Unclear risk

Baseline characteristics:

Quote: "expected prolonged duration of mechanical ventilation, $\mathrm{N}=12$ [Correction group] $\mathrm{N}=4$ [No correction group]; $\mathrm{P}=0.02 "$

Comment: differences in baseline characteristics have not been commented on by the study authors. The risk of material bias of this difference is unclear

Quote: "although a sample size of 152 patients was considered necessary to find a difference of $15 \%$ in bleeding between groups, the study was prematurely terminated. Recognition of the small amount of observed blood loss even in the "no correction" group resulted in an increasing resistance of the physicians to transfuse FFP and/or platelets in the "correction" group"

Comment: trial was stopped early due to increased resistance to recruitment and low rate of bleeding in either arm of the study 
ACCP: American College of Chest Physicians.

aPTT: activated partial thromboplastin time.

DIC: disseminated intravascular coagulation.

ERCP: endoscopic retrograde cholangiography.

FFP: fresh frozen plasma.

GCS: Glasgow Coma Scale.

$\mathrm{Hb}$ : haemoglobin.

INR: international normalised ratio.

ISRCTN: International Standard Randomized Controlled Trials Number.

ITU: intensive treatment unit.

NR: not reported.

NTR: Netherlands Trial Register

PDT: percutaneous dilatational tracheotomy

PLT: platelet.

PT: prothrombin time.

ROTEM: rotational thromboelastometry.

SCCM: Society of Critical Care Medicine.

SD: standard deviation.

SOC: standard of care.

TEG: thromboelastography.

Characteristics of excluded studies [ordered by study ID]

\begin{tabular}{|c|c|}
\hline Study & Reason for exclusion \\
\hline Bauer 1986 & The intervention did not meet our eligibility criteria (plasma transfusion to prevent bleeding) \\
\hline Cao 2016 & The participant population did not meet our eligibility criteria \\
\hline ChiCTR-INR-17013901 & The comparator did not meet our eligibility criteria \\
\hline Freeman 1998 & The comparator did not meet our eligibility criteria \\
\hline Galganski 2017 & The comparator did not meet our eligibility criteria \\
\hline Gazzard 1975 & The study design did not meet our eligibility criteria \\
\hline Hedstrand 1987 & $\begin{array}{l}\text { Timing of the intervention did not meet our eligibility criteria (before surgery or invasive proce- } \\
\text { dure) }\end{array}$ \\
\hline Hildebrandt 2007 & The study design did not meet our eligibility criteria \\
\hline Jilma-Stohlawetz 2011 & The participant population did not meet our eligibility criteria \\
\hline Kerner 2008 & The study design did not meet our eligibility criteria \\
\hline Laine 2003 & The comparator did not meet our eligibility criteria \\
\hline Lance 2012 & The participant population did not meet our eligibility criteria \\
\hline Lerner 1997 & The comparator did not meet our eligibility criteria \\
\hline Liu 1994 & $\begin{array}{l}\text { Timing of the intervention did not meet our eligibility criteria (before surgery or invasive proce- } \\
\text { dure) }\end{array}$ \\
\hline Mintz 2006 & The comparator did not meet our eligibility criteria \\
\hline NCT00233246 & The study was withdrawn before enrolment \\
\hline
\end{tabular}

Prophylactic plasma transfusion for patients without inherited bleeding disorders or anticoagulant use undergoing non-cardiac surgery 50 or invasive procedures (Review)

Copyright $\odot 2019$ The Cochrane Collaboration. Published by John Wiley \& Sons, Ltd. 


\begin{tabular}{|c|c|}
\hline Study & Reason for exclusion \\
\hline NCT00235183 & The comparator did not meet our eligibility criteria \\
\hline NCT00302965 & The participant population did not meet our eligibility criteria \\
\hline NCT00656396 & The intervention did not meet our eligibility criteria (plasma transfusion to prevent bleeding) \\
\hline NCT00994045 & $\begin{array}{l}\text { Timing of the intervention did not meet our eligibility criteria (before surgery or invasive proce- } \\
\text { dure) }\end{array}$ \\
\hline NCT02352181 & $\begin{array}{l}\text { Timing of the intervention did not meet our eligibility criteria (before surgery or invasive proce- } \\
\text { dure) }\end{array}$ \\
\hline NCT03700723 & The comparator did not meet our eligibility criteria \\
\hline Palmieri 2013 & The comparator did not meet our eligibility criteria \\
\hline Pieters 2015 & $\begin{array}{l}\text { Timing of the intervention did not meet our eligibility criteria (before surgery or invasive proce- } \\
\text { dure) }\end{array}$ \\
\hline Ramies 2002 & The comparator did not meet our eligibility criteria \\
\hline Rocha 2017 & The indication for the intervention did not meet our eligibility criteria \\
\hline Sommoggy 1990 & $\begin{array}{l}\text { Timing of the intervention did not meet our eligibility criteria (before surgery or invasive proce- } \\
\text { dure) }\end{array}$ \\
\hline Tinmouth 2008 & The comparator did not meet our eligibility criteria \\
\hline Urwyler 2009 & $\begin{array}{l}\text { Timing of the intervention did not meet our eligibility criteria (before surgery or invasive proce- } \\
\text { dure) }\end{array}$ \\
\hline Wang 2010 & $\begin{array}{l}\text { Timing of the intervention did not meet our eligibility criteria (before surgery or invasive proce- } \\
\text { dure) }\end{array}$ \\
\hline Wieding 1999 & The comparator did not meet our eligibility criteria \\
\hline Williamson 1999 & The comparator did not meet our eligibility criteria \\
\hline
\end{tabular}

\section{Characteristics of studies awaiting assessment [ordered by study ID]}

Boyd 1996

\begin{tabular}{ll} 
Study design: randomised controlled trial \\
Study grouping: parallel group \\
Masking: not reported \\
\hline Enrolment: number not reported \\
Included criteria: \\
Participants \\
- Over 18 years of age \\
- Bleeding time > 10 minutes (both FFP and conjugated oestrogen groups)
\end{tabular}


Boyd 1996 (Continued)

\section{Excluded criteria:}

- Patients receiving oestrogen treatment

- Patients receiving non-steroidal anti-inflammatory drugs

Interventions

\section{Intervention characteristics}

Conjugated oestrogen group

- Type of biological agent: conjugated oestrogen (Premarin, Wyeth Ayerst Laboratories, Philadelphia, PA)

- Dose of intervention: $50 \mathrm{mg}$

- Timing of intervention: following induction of anaesthesia*

FFP group

- Type of biological agent: FFP

- Dose of intervention: 2 units

- Timing of intervention: following induction of anaesthesia*

Control group

- Type of biological agent: none

Outcomes

\section{Main outcome measures:}

- Bleeding time measurements [time frame: end of surgery, at 24 hours, and at 48 hours]

- Other laboratory tests (creatinine, blood urea nitrogen, platelet count, prothrombin time, partial thromboplastin time, fibrinogen, and TEG values) [time frame: end of surgery, at 24 hours, and at 48 hours]

Notes

Sponsorship source: not reported

Country: United States of America

Setting: university regional referral centre for renal transplantation

Author's name: Gwendolyn L. Boyd

Institution: University of Alabama at Birmingham

Address: Department of Anesthesiology, University of Alabama at Birmingham, 619 19th St S, JT 845, Birmingham, AL 35233-6810

Trial registration: not reported

Date trial registered: not reported

Date trial started recruiting participants: not reported

Date trial completed recruitment: not reported

*It is unclear from the report whether the intervention was completed prior to the start of surgery. Inclusion or exclusion of this study is dependent on this information, which has been sought from the primary author (personal communication)"

Methods
Study design: randomised trial
Study grouping: parallel group

Prophylactic plasma transfusion for patients without inherited bleeding disorders or anticoagulant use undergoing non-cardiac surgery 52 or invasive procedures (Review)

Copyright $\odot 2019$ The Cochrane Collaboration. Published by John Wiley \& Sons, Ltd. 
Masking: open-label; blinded assessment of primary endpoint

Number of study centres: 2

Estimated $\mathbf{N}=\mathbf{4 6}$
Included criteria:
- Patient with spontaneous intracranial haemorrhage or traumatic intracranial
- tient requiring neurological surgery
- 18 years and older (adult, senior)
- Female or male
Excluded criteria:
- Concomitant use with oral anticoagulant drugs
- Acquired deficiency of coagulation factors whose treatment is established
- Hypersensitivity to a PCC
- History of thrombocytopenia induced by heparin
- Disseminated intravascular coagulation
- Extracranial active bleeding
- Hypersensitivity to vitamin $\mathrm{K}$

Interventions

Intervention characteristics

Experimental group

- Type of intervention: prothrombin complex concentrate

- Dose of intervention: administration of a single dose of prothrombin complex concentrate (25 U/ kg equivalent factor IX)

Active comparator

- Type of comparator: FFP

- Dose of comparator: administration of a single dose of fresh frozen plasma of $15 \mathrm{~mL} / \mathrm{kg}$ intervention

\section{Outcomes}

Primary outcomes: proportion of patients with correction of prothrombin time (PT > 60\%) [time frame: end of treatment administration (an average of 1 hour)]

Secondary outcomes: not described

\section{Country: France}

Setting: adult neurological patients with coagulopathy

Author's name: Laurence Salomon

Institution: Fondation Ophtalmologique Adolphe de Rothschild

Email: Isalomon@for.paris

Address: Fondation Ophtalmologique Adolphe de Rothschild, Paris, France 75019

Trial registration: NCT02777424

Date trial registered: 19 May 2016

Date trial started recruiting participants: January 2016

Prophylactic plasma transfusion for patients without inherited bleeding disorders or anticoagulant use undergoing non-cardiac surgery 
Tseĭmakh 2008

\begin{tabular}{ll}
\hline Methods & Comparative study \\
\hline Participants & Participants with pancreonecrosis, $\mathrm{N}=60$ \\
\hline Interventions & Cryosupernatant plasma vs fresh frozen plasma \\
\hline Outcomes & Laboratory indices, lethality, other outcomes \\
\hline Notes & $\begin{array}{l}\text { English translation of study abstract by study authors is available from study report, from which } \\
\text { the above information is taken. Full-text report in Russian. Currently awaiting translation of report } \\
\text { by Cochrane Russia }\end{array}$ \\
\hline
\end{tabular}

FFP: fresh frozen plasma.

PCC: Prothrombin complex concentrate

PT: prothrombin time.

TEG: thromboelastography.

Characteristics of ongoing studies [ordered by study ID]

\section{NCT02561026}

Trial name or title Transfusion of plasma prior to invasive procedures pilot trial (TOPPIT)

\begin{tabular}{|c|c|}
\hline Methods & $\begin{array}{l}\text { Study design: randomised controlled trial in } 3 \text { Canadian hospitals } \\
\text { Study grouping: parallel group } \\
\text { Masking: open-label }\end{array}$ \\
\hline Participants & $\begin{array}{l}\text { Estimated enrolment: } \mathrm{N}=80 \\
\text { Included criteria: } \\
\text { - Age } 18 \text { years to } 65 \text { years (adult) } \\
\text { - Admission or planned admission (e.g. patients in emergency department who are being seen by } \\
\text { the ICU team) to an intensive care unit } \\
\text { - Elevated INR between } 1.5 \text { and } 2.5 \\
\text { - Requiring an invasive procedure in the next } 24 \text { hours including central venous line, arterial line, } \\
\text { paracentesis, thoracocentesis, bronchoscopy, endoscopy, and ultrasound-guided biopsy (mass } \\
\text { or organ) or fluid drainage } \\
\text { - Female or male } \\
\text { Excluded criteria: } \\
\text { - Active bleeding, defined as visible or suspected blood loss in last } 48 \text { hours, resulting in a fall in } \\
\text { haemoglobin } \geq 20 \text { g/L, requiring a red cell transfusion or an intervention to control bleeding } \\
\text { - Full-dose therapeutic anticoagulation with warfarin, heparin, low molecular weight heparin, or } \\
\text { other novel oral anticoagulants } \\
\text { - Congenital bleeding disorders including haemophilia, von Willebrand disease, or platelet function } \\
\text { disorders } \\
\text { - Acquired coagulation factor deficiencies } \\
\text { - Frozen plasma transfusion during this ICU admission }\end{array}$ \\
\hline
\end{tabular}


- Use of other haemostatic blood products (recombinant factor VIla, prothrombin complex concentrate, cryoprecipitate, fibrinogen concentrate) during the ICU admission

- Previous enrolment in the study

Patients will not be excluded for thrombocytopenia nor for anti-platelet drugs. As a pilot trial for a pragmatic large randomised controlled trial, both thrombocytopenic patients and patients on anti-platelet agents will be enrolled as they are routinely encountered in clinical practice. Specific therapy (i.e. platelet transfusions) will not be mandated but will be left to local routine practice. Information regarding platelet counts, anti-platelet medications, platelet transfusions, and other haemostatic therapies will be collected

Interventions

\section{Intervention characteristics}

Experimental group:

- Type of intervention: frozen plasma transfusion

- Dose of intervention: not described

- Timing of intervention: before an invasive procedure

Comparator group

- Type of intervention: no FP transfusion

\section{Outcomes}

\section{Primary outcomes:}

- Recruitment feasibility, as measured by the number of participants screened per month at each centre [time frame: monthly, up to 21 months]

\section{Secondary outcomes:}

- Bleeding assessment (changes in haemoglobin and red cell transfusions as measured by a standardised bleeding assessment tool) [time frame: 24 to 48 hours post procedure]

- Ventilator requirement [time frame: 24 to 48 hours post frozen plasma transfusion] Requirement for mechanical ventilation as it pertains to the feasibility of study procedures

- Overall length of stay [time frame: length of stay will be measured as the number of days elapsed between hospital admission and hospital discharge dates up to 21 months]

- ICU length of stay [time frame: ICU length of stay will be measured as the number of days elapsed between intensive care unit admission and discharge dates up to 21 months]

\section{Starting date}

Contact information
January 2016

Sponsorship source: Ottawa Hospital Research Institute

\section{Country: Canada}

Setting: ICU non-bleeding patients with a coagulopathy requiring an invasive procedure

Authors name: Alan Tinmouth

Institution: Ottawa Hospital

Email: echatelain@ohri.ca

Address: The Ottawa Hospital, Ottawa Hospital Research Institute, Ottawa, Ontario, Canada, K1H8L6

Trial registration: NCT02561026 ID20150215-01H

Date trial registered: 25 September 2015

Date trial started recruiting participants: January 2016 


\begin{tabular}{|c|c|}
\hline Methods & $\begin{array}{l}\text { Study design: randomised controlled trial } \\
\text { Study grouping: parallel group } \\
\text { Masking: single-blinded (outcomes assessor) }\end{array}$ \\
\hline Participants & $\begin{array}{l}\text { Estimated enrolment: } \mathrm{N}=110 \\
\text { Included criteria: } \\
\text { - INR level between } 1.50 \text { and } 2.50 \text { inclusive } \\
\text { - Undergoing an invasive procedure at the bedside, at an endoscopy laboratory, or in radiology } \\
\text { - } 21 \text { years and older (adult, senior) } \\
\text { - Female or male } \\
\text { Excluded criteria: } \\
\text { - Undergoing a surgical procedure in the operating room } \\
\text { - Active bleeding } \\
\text { - Undergoing a procedure involving or proximal to the central nervous system or spinal cord } \\
\text { - Cardiac catheterisation } \\
\text { - Using } 4 \text { factor plasma concentrates } \\
\text { - Using systemic heparin/heparinoid therapy, direct factor } \mathrm{X} \text { inhibitors, and other anticoagulants } \\
\text { - Por which plasma will not correct prolonged INR } \\
\text { - Congenital coagulation disorders } \\
\text { - Acquired disorders (i.e. lupus anticoagulant) for which plasma will not correct the disorder } \\
\text { - Women who are pregnant } \\
\text { - Unwillingness to consider blood transfusion }\end{array}$ \\
\hline
\end{tabular}

\section{Intervention characteristics}

Experimental group

- Type of intervention: FFP transfusion

- Dose of intervention: $15 \mathrm{cc} / \mathrm{kg}$, range 10 to $20 \mathrm{cc} / \mathrm{kg}$, to a maximum of 5 units

- Timing of intervention: pre-procedure

Comparator group

- Type of intervention: no transfusions before the procedure

\section{Primary outcomes:}

- Change in haemoglobin level [time frame: within 2 days post procedure] (change from pre-procedure haemoglobin)

- Trial feasibility (rates of eligible and enrolled study participants) [time frame: 16 months study enrolment] 


\section{Secondary outcomes:}

- Red blood cell transfusion [time frame: within 2 days post procedure] (differences in number of units of red blood cell transfusions between the 2 study arms)

- Transfusion-associated cardiac overload (TACO) [time frame: within 2 days post procedure] (difference in rates between the 2 study arms)

- Transfusion-related acute lung injury (TRALI) [time frame: within 2 days post procedure] (difference in rates between the 2 study arms)

- Major bleed [time frame: within 2 days post procedure] ( $2 \mathrm{~g} / \mathrm{dL}$ or greater fall in haemoglobin level)

- Change in INR level post procedure [time frame: day of procedure] (change from pre-procedure INR level)

- Change in INR level day 1 [time frame: day 1 post procedure] (change from pre-procedure INR level)

- Change in INR level day 2 [time frame: day 2 post procedure] (change from pre-procedure INR level)

- Mortality [time frame: in-hospital up to 30 days] (death)

- Infection [time frame: within 2 days post procedure] (pneumonia or bloodstream infection)

- ICU admission [time frame: within 2 days post procedure] (new admission to the intensive care unit)

Starting date January 2016

Contact information

Sponsorship source: Rutgers, The State University of New Jersey

Country:

Setting:

Author's name: Jeffrey L Carson, Helaine Noveck

Institution: Johns Hopkins University, The Johns Hopkins Hospital. Robert Wood Johnson University Hospital

Email: jeffrey.carson@rutgers.edu.helaine.noveck@rutgers.edu

Address: Johns Hopkins University, The Johns Hopkins Hospital, Baltimore, Maryland, United States, 21287

Trial registration: NCT02637427 Pro20150001801 U.S. NIH Grant/Contract: 1R34HL125804-01A1

Date trial registered: 22 December 2015

Date trial started recruiting participants: January 2016

Estimated date trial completes recruitment: April 2020

Notes

\section{Smart 2017}

Trial name or title A prospective, randomised clinical trial comparing blood product use, bleeding events, and cost during and after endoscopic procedures in patients with cirrhosis and coagulopathy: rotational thromboelastometry (ROTEM) versus conventional therapy

Methods

Study design: randomised controlled trial

Study grouping: parallel group

Prophylactic plasma transfusion for patients without inherited bleeding disorders or anticoagulant use undergoing non-cardiac surgery 
Smart 2017 (Continued)

Participants
Enrolment: $\mathrm{N}=41$

\section{Included criteria:}

- Cirrhosis

- $\quad$ INR $>1.5$ and/or platelet count $<50,000 / \mu \mathrm{L}$

- Undergoing endoscopic procedure
Interventions

\section{Intervention characteristics}

Experimental group

- Type of intervention: ROTEM guided transfusion

- Dose of intervention: platelet, cryoprecipitate, and FFP transfusion determined by ROTEM tests

- Timing of intervention: before and after endoscopy

Comparator group

- Type of intervention: conventional coagulation-guided transfusion

- Dose of intervention: platelet, cryoprecipitate, and FFP transfusion determined by aPTT, PT, INR, platelet count, and fibrinogen tests

- Timing of intervention: before and after endoscopy

Outcomes included:

- Intra-procedural and post-procedural blood loss

- Types and numbers of blood products transfused

- Complications

- Cost

\section{Results}

- ROTEM group participants were transfused significantly lower volumes of blood products compared to conventional group participants ( $309 \mathrm{~mL}$ vs $461 \mathrm{~mL} ; \mathrm{P}=0.049$ )

- No statistically significant difference in blood loss and bleeding events between 2 groups

- More participants in the ROTEM group received cryoprecipitate compared with the conventional group ( $65 \%$ vs $19 \%$; $P=0.003$ )

- More participants in the conventional group received FFP than in the ROTEM group (62\% vs $10 \%$; $P=0.001)$

- Platelet use was equivalent between groups

- Total transfusion costs were higher in the ROTEM group than in the conventional group $(576.41$ vs 317.68; $P=0.045$ )

Starting date Completed

Contact information

Setting: participants with cirrhosis undergoing endoscopic procedures

Authors' names: Smart L, Wellner M, Gray N, Michaels A, Kirkpatrick R, Conteh L, Mumtaz K, Hanje A

Institution: University of Louisville, Louisville (Smart, Wellner); The Ohio State University Wexner Medical Center, Columbus, America (Gray, Michaels, Kirkpatrick, Conteh, Mumtaz, Hanje)

Notes

Written abstract from 68th Annual Meeting of the American Association for the Study of Liver Diseases, 2017

Citation:

Smart L, Wellner M, Gray N, Michaels A, Kirkpatrick R, Conteh L, et al. A prospective, randomised clinical trial comparing blood product use, bleeding events, and cost during and after endoscopic

Prophylactic plasma transfusion for patients without inherited bleeding disorders or anticoagulant use undergoing non-cardiac surgery 58 or invasive procedures (Review)

Copyright ( 2019 The Cochrane Collaboration. Published by John Wiley \& Sons, Ltd. 
aPTT: activated partial thromboplastin time.

FFP: fresh frozen plasma.

FP: frozen plasma.

ICU: intensive care unit.

INR: international normalised ratio.

PT: prothrombin time.

ROTEM: rotational thromboelastometry.

TACO: transfusion-associated circulatory overload.

TRALI: transfusion-related acute lung injury.

\section{DATA AND ANALYSES}

Comparison 1. Prophylactic plasma transfusion before surgery versus no prophylactic plasma transfusion before surgery (colloid, crystalloid, placebo, or no treatment)

\begin{tabular}{lllll}
\hline Outcome or subgroup title & $\begin{array}{l}\text { No. of } \\
\text { studies }\end{array}$ & $\begin{array}{l}\text { No. of } \\
\text { partici- } \\
\text { pants }\end{array}$ & Statistical method & Effect size \\
\hline 1 All-cause mortality & 2 & Risk Ratio (M-H, Fixed, 95\% Cl) & Totals not selected \\
\hline 1.1 Up to 24 hours & 1 & Risk Ratio (M-H, Fixed, 95\% Cl) & $0.0[0.0,0.0]$ \\
\hline 1.2 Up to 30 days & 1 & Risk Ratio (M-H, Fixed, 95\% Cl) & $0.0[0.0,0.0]$ \\
\hline 2 Major bleeding & 2 & Risk Ratio (M-H, Fixed, 95\% Cl) & Totals not selected \\
\hline 2.1 Within 24 hours & 2 & Risk Ratio (M-H, Fixed, 95\% Cl) & $0.0[0.0,0.0]$
\end{tabular}

Analysis 1.1. Comparison 1 Prophylactic plasma transfusion before surgery versus no prophylactic plasma transfusion before surgery (colloid, crystalloid, placebo, or no treatment), Outcome 1 All-cause mortality.

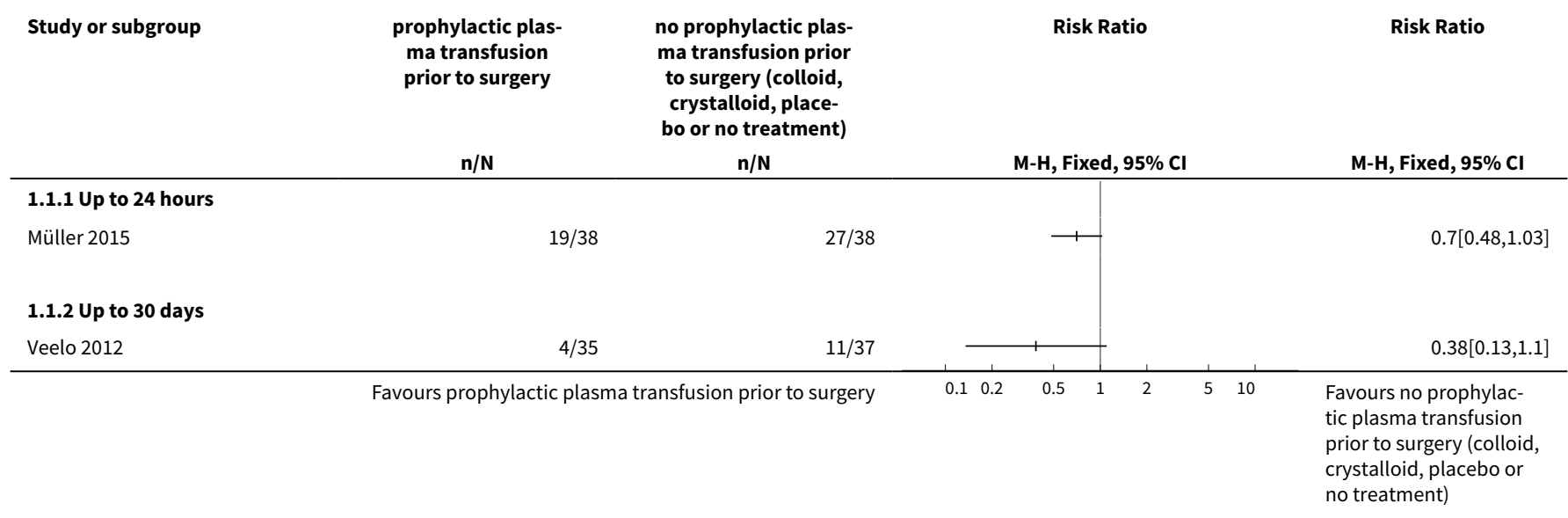

Prophylactic plasma transfusion for patients without inherited bleeding disorders or anticoagulant use undergoing non-cardiac surgery 
Analysis 1.2. Comparison 1 Prophylactic plasma transfusion before surgery versus no prophylactic plasma transfusion before surgery (colloid, crystalloid, placebo, or no treatment), Outcome 2 Major bleeding.

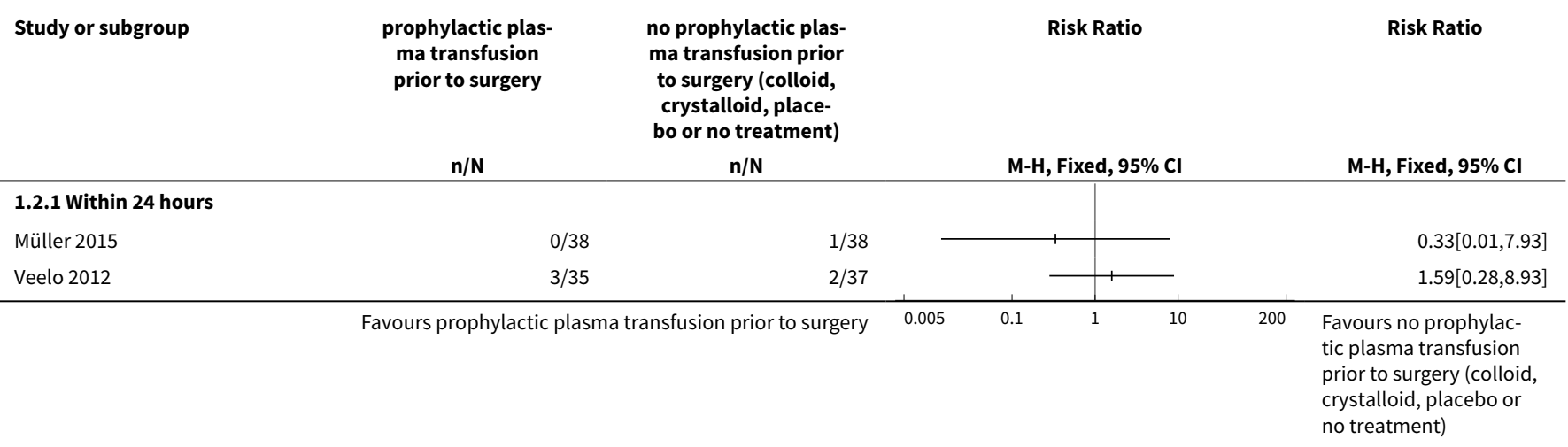

Comparison 2. Prophylactic plasma transfusion before surgery versus alternative haemostatic agents

\begin{tabular}{lllll}
\hline Outcome or subgroup title & $\begin{array}{l}\text { No. of } \\
\text { studies }\end{array}$ & $\begin{array}{l}\text { No. of } \\
\text { partici- } \\
\text { pants }\end{array}$ & Statistical method & Effect size \\
\hline $\begin{array}{l}\text { 1 Serious adverse event due to plasma } \\
\text { transfusion }\end{array}$ & 1 & Risk Ratio (M-H, Fixed, 95\% Cl) & Totals not selected \\
\hline $\begin{array}{l}1.1 \text { Within } 24 \text { hours } \\
2 \text { Coagulation test abnormalities }\end{array}$ & 1 & Risk Ratio (M-H, Fixed, 95\% Cl) & $0.0[0.0,0.0]$ \\
\hline $\begin{array}{l}2.1 \text { Within } 24 \text { hours, correction of par- } \\
\text { tial thromboplastin time }\end{array}$ & 1 & Risk Ratio (M-H, Fixed, 95\% Cl) & Totals not selected \\
\hline $\begin{array}{l}2.2 \text { Within } 24 \text { hours, correction of pro- } \\
\text { thrombin time }\end{array}$ & 1 & Risk Ratio (M-H, Fixed, 95\% Cl) & $0.0[0.0,0.0]$ \\
\hline $\begin{array}{l}2.3 \text { Within } 24 \text { hours, correction of Nor- } \\
\text { motest }\end{array}$ & 1 & Risk Ratio (M-H, Fixed, 95\% Cl) & $0.0[0.0,0.0]$ \\
\hline
\end{tabular}

Analysis 2.1. Comparison 2 Prophylactic plasma transfusion before surgery versus alternative haemostatic agents, Outcome 1 Serious adverse event due to plasma transfusion.

\begin{tabular}{|c|c|c|c|c|c|c|c|}
\hline \multirow{2}{*}{$\begin{array}{l}\text { Study or subgroup } \\
2.1 .1 \text { Within } 24 \text { hours }\end{array}$} & \multirow{2}{*}{$\begin{array}{c}\text { prophylactic plas- } \\
\text { ma transfusion } \\
\text { prior to surgery } \\
\mathrm{n} / \mathrm{N}\end{array}$} & \multirow{2}{*}{$\begin{array}{c}\text { alternative haemo- } \\
\text { static agents } \\
\text { n/N }\end{array}$} & \multicolumn{4}{|c|}{ Risk Ratio } & \multirow{2}{*}{$\begin{array}{c}\text { Risk Ratio } \\
\text { M-H, Fixed, } 95 \% \mathrm{Cl} \\
\end{array}$} \\
\hline & & & \multicolumn{3}{|c|}{ M-H, Fixed, $95 \% \mathrm{Cl}$} & & \\
\hline \multirow[t]{2}{*}{ Mannucci 1976} & $4 / 10$ & $0 / 11$ & & & & & $9.82[0.59,162.24]$ \\
\hline & Favours prophylactic plasn & רsfusion prior to surgery & 0.002 & 0.1 & 10 & 500 & $\begin{array}{l}\text { Favours alternative } \\
\text { haemostatic agents }\end{array}$ \\
\hline
\end{tabular}


Analysis 2.2. Comparison 2 Prophylactic plasma transfusion before surgery versus alternative haemostatic agents, Outcome 2 Coagulation test abnormalities.

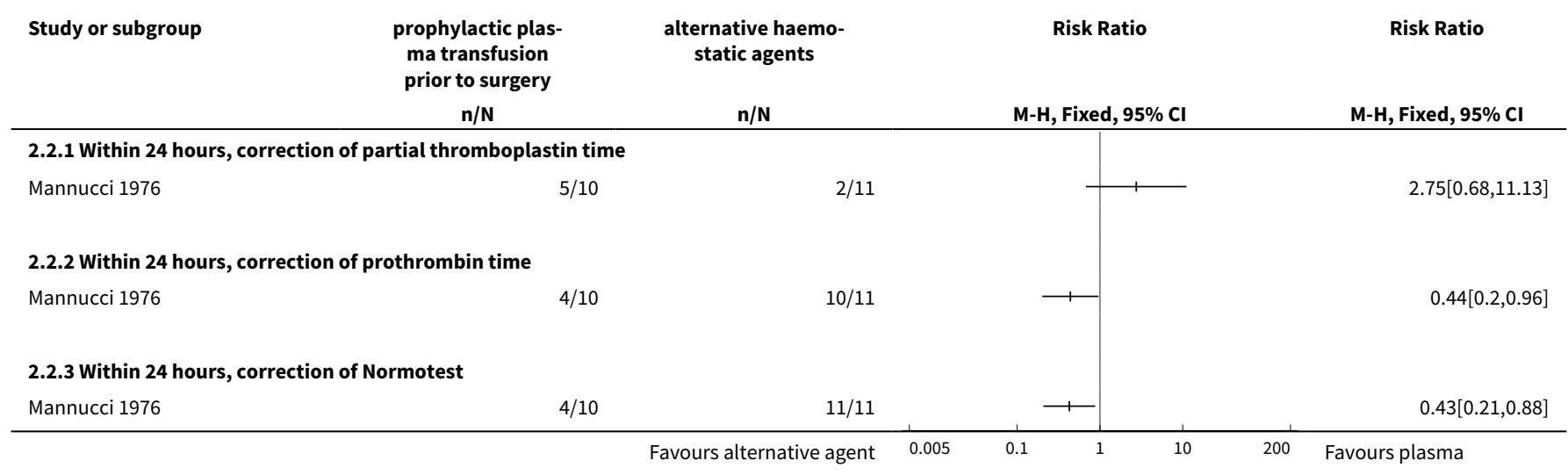

Comparison 3. Thromboelastography threshold versus standard of care (laboratory parameters)

\begin{tabular}{|c|c|c|c|c|}
\hline Outcome or subgroup title & $\begin{array}{l}\text { No. of } \\
\text { studies }\end{array}$ & $\begin{array}{l}\text { No. of } \\
\text { partici- } \\
\text { pants }\end{array}$ & Statistical method & Effect size \\
\hline 1 All-cause mortality & 1 & & $\begin{array}{l}\text { Risk Ratio (M-H, Fixed, 95\% } \\
\mathrm{Cl})\end{array}$ & Totals not selected \\
\hline 1.1 Up to 90 days & 1 & & $\begin{array}{l}\text { Risk Ratio (M-H, Fixed, 95\% } \\
\mathrm{Cl} \text { ) }\end{array}$ & $0.0[0.0,0.0]$ \\
\hline 2 Major bleeding & 1 & & $\begin{array}{l}\text { Risk Ratio (M-H, Fixed, 95\% } \\
\mathrm{Cl} \text { ) }\end{array}$ & Totals not selected \\
\hline 2.1 Within 24 hours & 1 & & $\begin{array}{l}\text { Risk Ratio (M-H, Fixed, 95\% } \\
\mathrm{Cl} \text { ) }\end{array}$ & $0.0[0.0,0.0]$ \\
\hline 2.2 Within 7 days & 1 & & $\begin{array}{l}\text { Risk Ratio (M-H, Fixed, 95\% } \\
\mathrm{Cl} \text { ) }\end{array}$ & $0.0[0.0,0.0]$ \\
\hline 3 Number of individuals requiring a transfusion & 1 & & $\begin{array}{l}\text { Risk Ratio (M-H, Fixed, 95\% } \\
\mathrm{Cl} \text { ) }\end{array}$ & Totals not selected \\
\hline 3.1 Within 7 days, overall blood product use & 1 & & $\begin{array}{l}\text { Risk Ratio (M-H, Fixed, 95\% } \\
\mathrm{Cl} \text { ) }\end{array}$ & $0.0[0.0,0.0]$ \\
\hline 3.2 Within 7 days, FFP use only & 1 & & $\begin{array}{l}\text { Risk Ratio (M-H, Fixed, 95\% } \\
\mathrm{Cl} \text { ) }\end{array}$ & $0.0[0.0,0.0]$ \\
\hline 3.3 Within 7 days, PLT use only & 1 & & $\begin{array}{l}\text { Risk Ratio (M-H, Fixed, 95\% } \\
\mathrm{Cl})\end{array}$ & $0.0[0.0,0.0]$ \\
\hline 3.4 Within 7 days, FFP and PLT use & 1 & & $\begin{array}{l}\text { Risk Ratio (M-H, Fixed, 95\% } \\
\text { Cl) }\end{array}$ & $0.0[0.0,0.0]$ \\
\hline $\begin{array}{l}4 \text { Serious adverse event due to plasma transfu- } \\
\text { sion }\end{array}$ & 1 & & $\begin{array}{l}\text { Risk Ratio (M-H, Fixed, 95\% } \\
\text { Cl) }\end{array}$ & Totals not selected \\
\hline
\end{tabular}

Prophylactic plasma transfusion for patients without inherited bleeding disorders or anticoagulant use undergoing non-cardiac surgery 


\begin{tabular}{lllll}
\hline Outcome or subgroup title & $\begin{array}{l}\text { No. of } \\
\text { studies }\end{array}$ & $\begin{array}{l}\text { No. of } \\
\text { partici- } \\
\text { pants }\end{array}$ & Statistical method & Effect size \\
\hline 4.1 Within 24 hours & 1 & & $\begin{array}{l}\text { Risk Ratio (M-H, Fixed, 95\% } \\
\mathrm{Cl})\end{array}$ & $0.0[0.0,0.0]$ \\
\hline
\end{tabular}

Analysis 3.1. Comparison 3 Thromboelastography threshold versus standard of care (laboratory parameters), Outcome 1 All-cause mortality.

\begin{tabular}{|c|c|c|c|c|c|c|c|}
\hline \multirow[t]{2}{*}{ Study or subgroup } & $\begin{array}{l}\text { thromboelastog- } \\
\text { raphy threshold }\end{array}$ & $\begin{array}{l}\text { standard of care (lab- } \\
\text { oratory parameters) }\end{array}$ & \multicolumn{4}{|c|}{ Risk Ratio } & \multirow{2}{*}{$\begin{array}{c}\text { Risk Ratio } \\
\text { M-H, Fixed, } 95 \% \mathrm{Cl} \\
\end{array}$} \\
\hline & $n / N$ & $n / N$ & \multicolumn{4}{|c|}{ M-H, Fixed, $95 \% \mathrm{Cl}$} & \\
\hline \multirow[t]{2}{*}{ De Pietri 2016} & $8 / 30$ & $7 / 30$ & & & 1 & & $1.14[0.47,2.75]$ \\
\hline & Favours th & boelastography threshold & 0.05 & 0.2 & 1 & 20 & $\begin{array}{l}\text { Favours standard of care } \\
\text { (laboratory parameters) }\end{array}$ \\
\hline
\end{tabular}

\section{Analysis 3.2. Comparison 3 Thromboelastography threshold versus} standard of care (laboratory parameters), Outcome 2 Major bleeding.

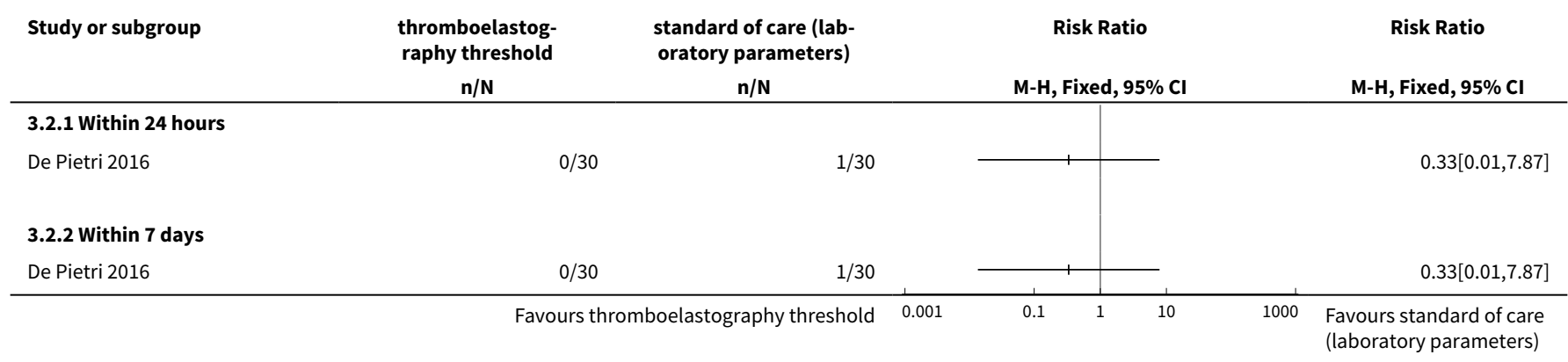

Analysis 3.3. Comparison 3 Thromboelastography threshold versus standard of care (laboratory parameters), Outcome 3 Number of individuals requiring a transfusion.

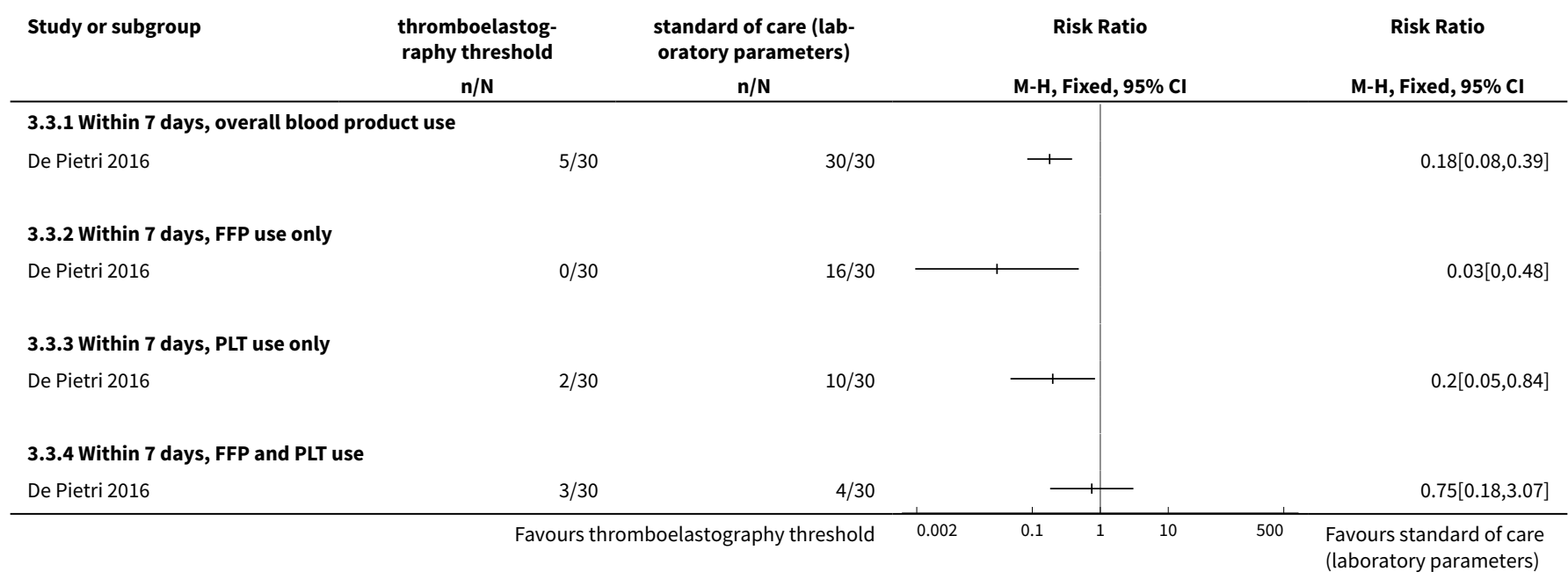

Prophylactic plasma transfusion for patients without inherited bleeding disorders or anticoagulant use undergoing non-cardiac surgery 62 or invasive procedures (Review)

Copyright (C) 2019 The Cochrane Collaboration. Published by John Wiley \& Sons, Ltd. 
Analysis 3.4. Comparison 3 Thromboelastography threshold versus standard of care (laboratory parameters), Outcome 4 Serious adverse event due to plasma transfusion.

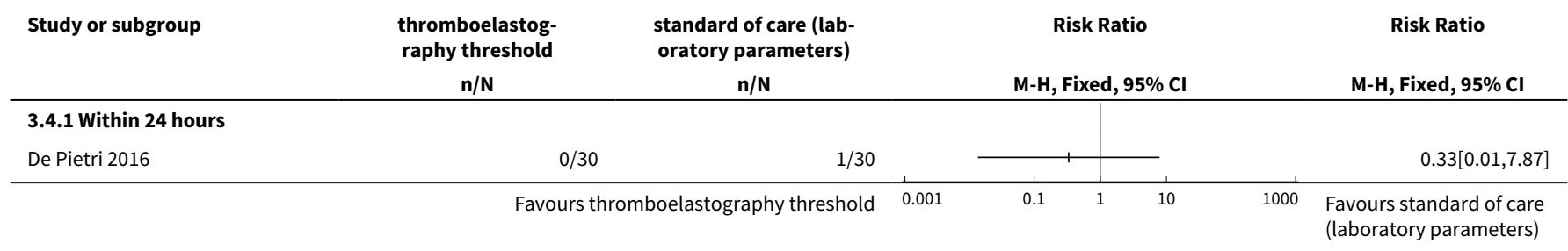

\section{ADDITIONAL TABLES}

Prophylactic plasma transfusion for patients without inherited bleeding disorders or anticoagulant use undergoing non-cardiac surgery 


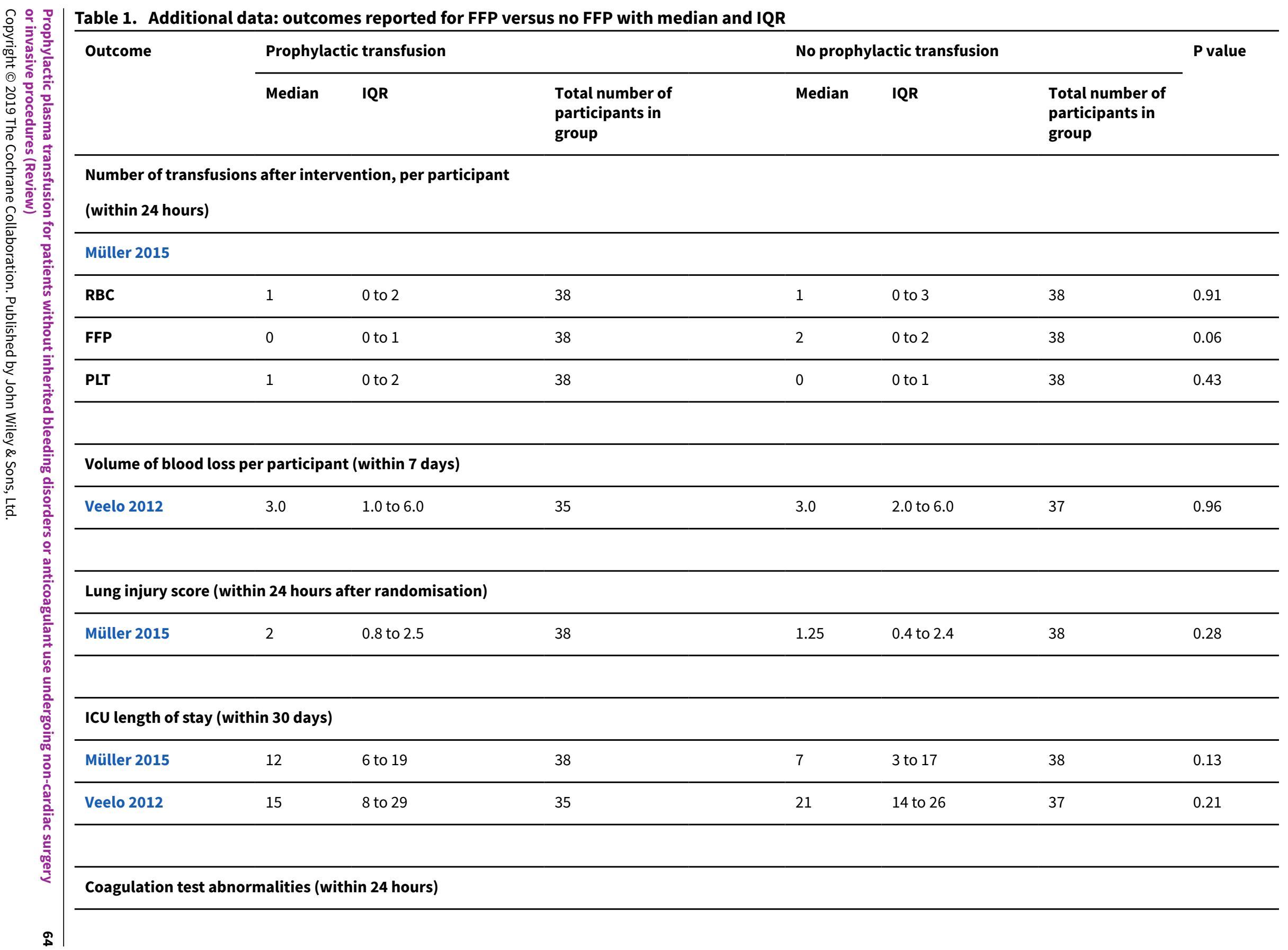




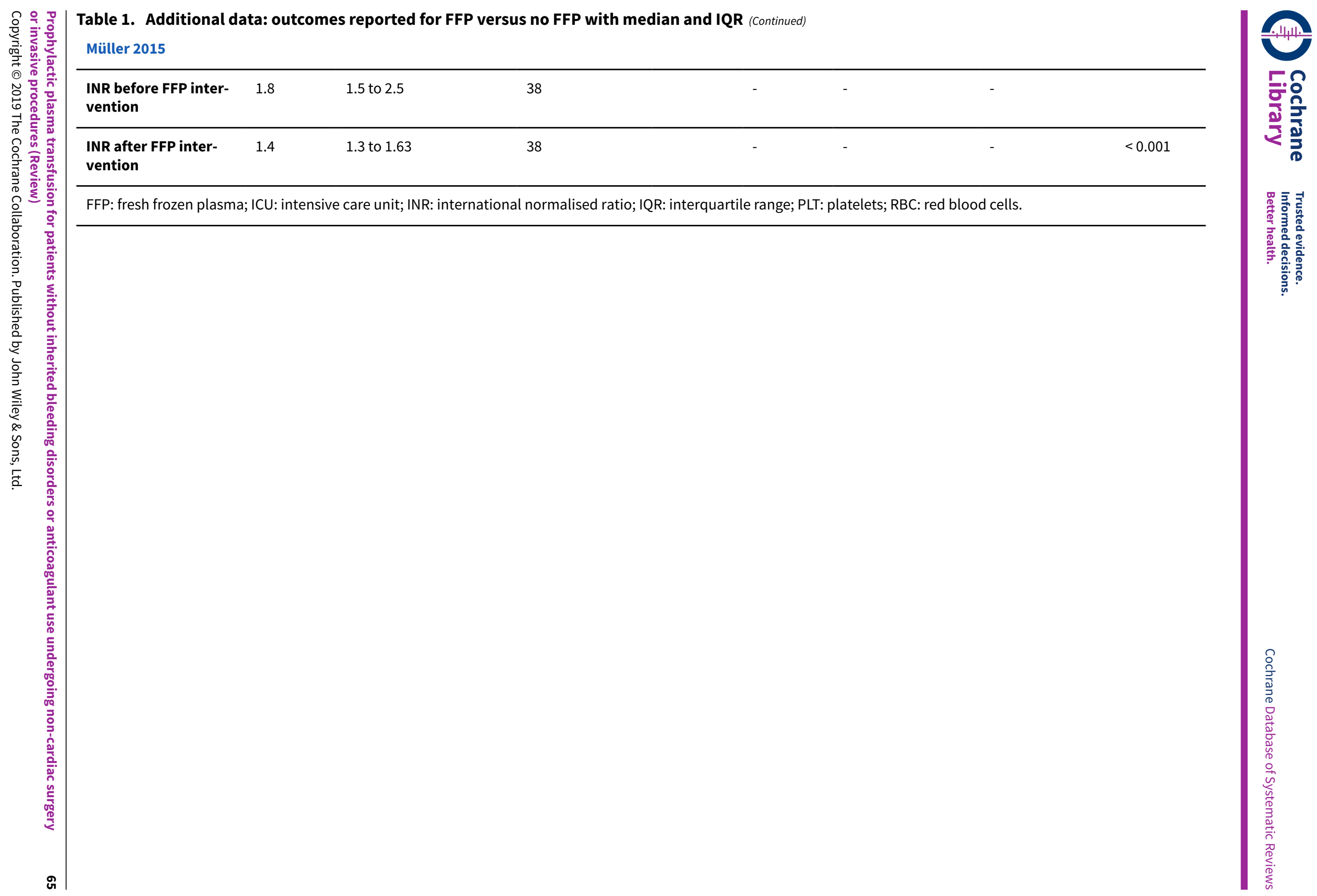




\title{
A P PE N DICES
}

\section{Appendix 1. Definition of 'surgery', American College of Surgeons}

In conjunction with the section "Classification of procedures" in the International Classification of Diseases, Ninth Revision, Clinical Modification (ICD9-CM 2011), we have used the following definition of 'surgery' from the American College of Surgeons (ACS 2007), to assist with determination of studies for inclusion.

"Surgery is performed for the purpose of structurally altering the human body by incision or destruction of tissues and is part of the practice of medicine. Surgery also is the diagnostic or therapeutic treatment of conditions or disease processes by any instruments causing localized alteration or transportation of live human tissue, which include lasers, ultrasound, ionizing radiation, scalpels, probes, and needles. The tissue can be cut, burned, vaporized, frozen, sutured, probed, or manipulated by closed reduction for major dislocations and fractures, or otherwise altered by any mechanical, thermal, light-based, electromagnetic, or chemical means. Injection of diagnostic or therapeutic substances into body cavities, internal organs, joints, sensory organs, and the central nervous system is also considered to be surgery (this does not include administration by nursing personnel of some injections, such as subcutaneous, intramuscular, and intravenous when ordered by a physician). All of these surgical procedures are invasive, including those that are performed with lasers, and the risks of any surgical intervention are not eliminated by using a light knife or laser in place of a metal knife or scalpel. Patient safety and quality of care are paramount, and the College therefore believes that patients should be assured that individuals who perform these types of surgery are licensed physicians (defined as doctors of medicine or osteopathy) who meet appropriate professional standards."

\section{Appendix 2. Definition of serious adverse event}

We used a definition of 'Serious Adverse Event' based on guidance from Chou (Chou 2010), the International Conference on Harmonisation (ICH) of Technical Requirements for Registration of Pharmaceuticals for Human Use (ICH 1994), the International Society of Blood Transfusion (ISBT) (ISBT 2011), and the United States of America Government (U.S. Government 2016).

Any undesirable medical occurrence in an individual administered a pharmaceutical, medical, or biological product, or undergoing an intervention, that:

- results in death;

- is life-threatening;

- requires hospitalisation or prolongation of existing hospitalisation;

- results in persistent or significant disability/incapacity;

- is a congenital anomaly/birth defect; or

- is an important medical event that may not fulfil the criteria above, but may jeopardise the individual and may require medical or surgical intervention to prevent the criteria above.

\section{Appendix 3. CENTRAL search strategy}

\#1 MeSH descriptor: [Plasma] this term only \#2 MeSH descriptor: [Blood Component Transfusion] this term only \#3 plasma

\#4 \#2 and \#3

\#5 plasma:ti,ab near/5 (transfus*:ti,ab or prophyla*:ti,ab or fresh*:ti,ab or frozen:ti,ab or freez*:ti,ab or prefrozen:ti,ab or prefreez*:ti,ab or thaw*:ti,ab or prethaw*:ti,ab or liquid:ti,ab or infus*:ti,ab or treatment*:ti,ab or therap*:ti,ab or administ*:ti,ab or donor ${ }^{\star}: \mathrm{ti}^{\star}, \mathrm{ab}$ or donat $^{\star}: \mathrm{ti}, \mathrm{ab}$ or pasteurized:ti,ab or pasteurised:ti,ab or methylene:ti,ab or solvent:ti,ab or detergent:ti,ab or cryoprecipitate:ti,ab or supernatant:ti,ab or cryosupernatant:ti,ab)

\#6 (homolog* or allogen* or allo-gen*or universal or clinical or human or pooled or versus) next plasma

\#7 (FFP or SDFFP or MBFFP or F24 or FP24 or PF24 or MB-FFP or SD-FFP or uniplas* or octaplas* or lyoplas* or frischplasma or "plasma versus")

\#8 (plasma near/3 (pathogen* or unit* or ratio*))

$9 \# 1$ or \#4 or \#5 or \#6 or \#7 or \#8

\#10 MeSH descriptor: [Specialties, Surgical] explode all trees

\#11 MeSH descriptor: [Surgical Procedures, Operative] explode all trees

$\# 12$ surg $^{\star}$ or presurg ${ }^{\star}$ or postsurg ${ }^{\star}$ or operat ${ }^{\star}$ or preoperat ${ }^{\star}$ or perioperat ${ }^{\star}$ or postoperat ${ }^{\star}$ or transplant ${ }^{\star}$

\#13 \#10 or \#11 or \#12

$\# 14 \# 9$ and \#13

\section{Appendix 4. MEDLINE search strategy}

\author{
1. *Plasma/ \\ 2. Plasma/ and transfus*.mp. \\ 3. Blood Component Transfusion/ and plasma.mp.
}

Prophylactic plasma transfusion for patients without inherited bleeding disorders or anticoagulant use undergoing non-cardiac surgery or invasive procedures (Review)

Copyright (c) 2019 The Cochrane Collaboration. Published by John Wiley \& Sons, Ltd. 
4. (plasma adj5 (transfus* or prophyla* or fresh* or frozen or freez ${ }^{\star}$ or prefrozen or prefreez ${ }^{\star}$ or thaw ${ }^{\star}$ or prethaw ${ }^{\star}$ or liquid or infus ${ }^{\star}$ or treatment ${ }^{\star}$ or therap* or administ* or donor ${ }^{\star}$ or donat* or pasteurized or pasteurised or methylene or solvent or detergent or cryoprecipitate or supernatant or cryosupernatant)).tw,kf.

5. ((homolog* or allogen* or allo-gen*or universal or clinical or human or pooled or versus) adj plasma).tw,kf.

6. (FFP or SDFFP or MBFFP or F24 or FP24 or PF24 or MB-FFP or SD-FFP or uniplas* or octaplas ${ }^{\star}$ or lyoplas ${ }^{\star}$ or frischplasma or plasma versus).tw,kf.

7. (plasma adj3 (pathogen* or unit* or ratio*)).tw,kf.

8. or/1-7

9. exp Specialties, Surgical/

10. exp Surgical Procedures, Operative/

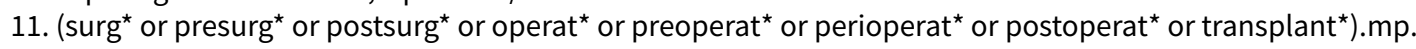

12. 9 or 10 or 11

13. Meta-Analysis.pt.

14. ((meta analy ${ }^{\star}$ or metaanaly $\left.{ }^{\star}\right)$ and (trials or studies)).ab.

15. (meta analy ${ }^{\star}$ or metaanaly* or evidence-based).ti.

16. ((systematic ${ }^{\star}$ or evidence-based) adj2 (review* ${ }^{\star}$ or overview $\left.\left.{ }^{\star}\right)\right)$.tw.

17. (cochrane or medline or pubmed or embase or cinahl or cinhal or lilacs or "web of science" or science citation index or scopus or search terms or literature search or electronic search* or comprehensive search ${ }^{\star}$ or systematic search ${ }^{\star}$ or published articles or search strateg $\left.{ }^{\star}\right) . a b$.

18. Cochrane Database of systematic reviews.jn.

19. (additional adj (papers or articles or sources)).ab.

20. ((electronic* or online) adj (sources or resources or databases)).ab.

21. (relevant adj (journals or articles)).ab.

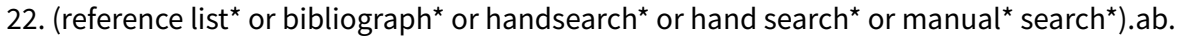

23. or/13-22

24. Review.pt.

25. RANDOMIZED CONTROLLED TRIALS AS TOPIC/

26. selection criteria.ab. or critical appraisal.ti.

27. (data adj (extraction or analys $\left.{ }^{\star}\right)$ ).ab.

28. RANDOMIZED CONTROLLED TRIALS/

29. or $/ 25-28$

30. 24 and 29

31.23 or 30

32. randomized controlled trial.pt.

33. controlled clinical trial.pt.

34. randomi ${ }^{\star}$.tw.

35. (placebo or randomly or groups).ab.

37. trial.tw.

38. or $/ 32-37$

39. 31 or 38

40. (ANIMALS/ or exp ANIMAL EXPERIMENTATION/ or exp MODELS, ANIMAL/) not HUMANS/

41. Editorial.pt.

42. 40 or 41

43.39 not 42

44. 8 and 12 and 43

\section{Appendix 5. Embase search strategy}

1. Plasma Transfusion/

2. Fresh Frozen Plasma/

3. Octaplas/

4. (plasma adj5 (transfus ${ }^{\star}$ or prophyla* or fresh* or frozen or freez ${ }^{\star}$ or prefrozen or prefreez ${ }^{\star}$ or thaw ${ }^{\star}$ or prethaw* or liquid or infus or treatment* or therap* or administ* or donor ${ }^{\star}$ or donat* or pasteurized or pasteurised or methylene or solvent or detergent or cryoprecipitate or supernatant or cryosupernatant)).tw.

5. ((homolog* or allogen* or allo-gen*or universal or human or pooled or clinical or versus) adj plasma).tw.

6. (FFP or SDFFP or MBFFP or F24 or FP24 or PF24 or MB-FFP or SD-FFP or uniplas* or octaplas* or lyoplas* or frischplasma or plasma versus).tw.

7. (plasma adj3 (pathogen ${ }^{\star}$ or unit ${ }^{\star}$ or ratio $\left.{ }^{\star}\right)$ ).tw.

8. or/1-7

9. exp Surgery/

10. surg $^{\star}$ or presurg $^{\star}$ or postsurg ${ }^{\star}$ or operat $^{\star}$ or preoperat $^{\star}$ or perioperat ${ }^{\star}$ or postoperat $^{\star}$ or transplant $\left.{ }^{\star}\right) . \mathrm{mp}$.

11. or/9-10

12. 8 and 11

Prophylactic plasma transfusion for patients without inherited bleeding disorders or anticoagulant use undergoing non-cardiac surgery 
13. Meta Analysis/

14. (meta analy ${ }^{\star}$ or metaanaly* or evidence-based).ti.

15. ((meta analy ${ }^{\star}$ or metaanaly $\left.{ }^{\star}\right)$ and (trials or studies)).ab.

16. Systematic Review/

17. ((systematic ${ }^{\star}$ or evidence-based) adj2 (review* ${ }^{\star}$ or overview*)).tw.

18. (cochrane or medline or pubmed or embase or cinahl or cinhal or lilacs or "web of science" or science citation index or scopus or search terms or literature search or electronic search* or comprehensive search* or systematic search* or published articles or search strateg* or reference list* or bibliograph* or handsearch* or hand search* or manual* search*).ab.

19. ((electronic* or online) adj (sources or resources or databases)).ab.

20. ((additional adj (papers or articles or sources)) or (relevant adj (journals or articles))).ab.

21. Review.pt. and (data extraction or selection criteria).ab.

22. or $/ 13-21$

23. Editorial.pt.

24. 22 not 23

25. crossover-procedure/ or double-blind procedure/ or randomized controlled trial/ or single-blind procedure/

26. (random* or factorial* or crossover* or cross over ${ }^{\star}$ or cross-over* or placebo* or doubl* blind* or singl* blind* or assign* or allocat* or volunteer $\left.{ }^{\star}\right) \cdot \mathrm{mp}$.

27. or $/ 24-26$

28. 12 and 27

29. limit 28 to embase

\section{Appendix 6. CINAHL search strategy}

S1 (MM "Plasma")

S2 (MH "Plasma")

S3 TX transfus*

S4 S2 AND S3

S5 (MH "Blood Component Transfusion")

S6 TX plasma

S7 S5 AND S6

S8 TI ( plasma N5 (transfus* or prophyla* or fresh* or frozen or freez ${ }^{\star}$ or prefrozen or prefreez ${ }^{\star}$ or thaw or prethaw $^{\star}$ or liquid or infus $^{\star}$ or treatment ${ }^{\star}$ or therap ${ }^{\star}$ or administ ${ }^{\star}$ or donor ${ }^{\star}$ or donat $^{\star}$ or pasteurized or pasteurised or methylene or solvent or detergent or cryoprecipitate or supernatant or cryosupernatant) ) OR AB ( plasma N5 (transfus* or prophyla* or fresh* or frozen or freez* or prefrozen or prefreez ${ }^{\star}$ or thaw ${ }^{\star}$ or prethaw ${ }^{\star}$ or infus ${ }^{\star}$ or treatment ${ }^{\star}$ or therap ${ }^{\star}$ or administ ${ }^{\star}$ or donor ${ }^{\star}$ or donat $^{\star}$ or pasteurized or pasteurised or methylene or solvent or detergent or cryoprecipitate or supernatant or cryosupernatant) )

S9 TI ( (homolog* or allogen* or allo-gen*or universal or clinical or human or pooled or versus or unit ${ }^{\star}$ ) N1 plasma ) OR AB ( (homolog ${ }^{\star}$ or allogen* or allo-gen* or universal or clinical or human or pooled or versus) N1 plasma )

S10 TX (FFP or SDFFP or MBFFP or F24 or FP24 or PF24 or MB-FFP or SD-FFP or uniplas* or octaplas* or lyoplas* or frischplasma or "plasma versus")

S11 TI ( plasma N3 (pathogen or unit* or ratio*) ) OR AB ( plasma N3 (pathogen inactivated or pathogen reduced or unit* or ratio*) )

S12 S1 OR S4 OR S7 OR S8 OR S9 OR S10 OR S11

S13 (MH "Specialties, Surgical+")

S14 (MH "Surgery, Operative+")

S15 TX (surg* or presurg ${ }^{\star}$ or postsurg* or operat* or preoperat ${ }^{\star}$ or perioperat ${ }^{\star}$ or postoperat ${ }^{\star}$ or transplant ${ }^{\star}$ )

S16 S13 OR S14 OR S15

S17 S12 AND S16

S18 (MH Clinical Trials+)

S19 PT Clinical Trial

S20 TI ((controlled trial $\left.{ }^{\star}\right)$ or (clinical trial $\left.\left.{ }^{\star}\right)\right)$ OR AB $\left(\left(\right.\right.$ controlled trial $\left.^{\star}\right)$ or (clinical trial $\left.\left.{ }^{\star}\right)\right)$

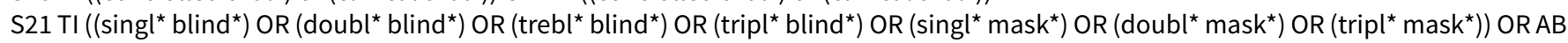

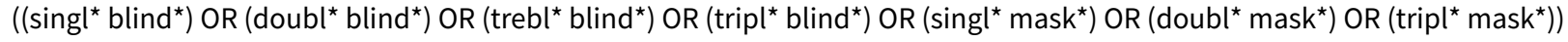

S22 $\mathrm{TI}$ randomi* OR AB randomi ${ }^{\star}$

S23 MH RANDOM ASSIGNMENT

S24 TI ((phase three) or (phase III) or (phase three)) or AB ((phase three) or (phase III) or (phase three))

S25 ( TI (random* N2 (assign* or allocat $\left.\left.{ }^{\star}\right)\right)$ ) OR ( AB (random N2 (assign^ or allocat $\left.\left.{ }^{\star}\right)\right)$ )

S26 MH PLACEBOS

S27 MH META ANALYSIS

S28 MH SYSTEMATIC REVIEW

S29 TI ("meta analys" OR metaanalys* OR "systematic review" OR "systematic overview" OR "systematic search*") OR AB ("meta analys OR metaanalys* OR "systematic review" OR "systematic overview" OR "systematic search^")

S30 TI ("literature review" OR "literature overview" OR "literature search*") OR AB ("literature review" OR "literature overview" OR "literature search*")

Prophylactic plasma transfusion for patients without inherited bleeding disorders or anticoagulant use undergoing non-cardiac surgery 
S31 TI (cochrane OR embase OR cinahl OR cinhal OR lilacs OR BIDS OR science AND citation AND index OR cancerlit) OR AB (cochrane OR embase OR cinahl OR cinhal OR lilacs OR BIDS OR science AND citation AND index OR cancerlit)

S32 TI placebo* OR AB placebo*

S33 MH QUANTITATIVE STUDIES

S34 S18 or S19 or S20 or S21 or S22 or S23 or S24 or S25 or S26 or S27 or S28 or S29 or S30 or S31 or S32 or S33

S35 S17 AND S34

\section{Appendix 7. PubMed search strategy}

\#1 (plasma[TIAB] AND (transfus*[TIAB] OR prophyla*[TIAB]))

\#2 "plasma therapy"[TIAB] OR "plasma treatment"[TIAB] OR "plasma administration"[TIAB] OR "fresh plasma"[TIAB] OR "frozen plasma" [TIAB] OR "prefrozen plasma"[TIAB] OR "thawed plasma"[TIAB] OR "prethawed plasma"[TIAB] OR "liquid plasma"[TIAB] OR "infused plasma"[TIAB] OR "plasma infused"[TIAB] OR "donated plasma"[TIAB] OR "donor plasma"[TIAB] OR "pasteurised plasma"[TIAB] OR "pasteurized plasma" [TIAB] OR "freeze-dried plasma"[TIAB] OR "methylene blue plasma"[TIAB] OR "solvent-detergent plasma"[TIAB] OR "supernatant plasma"[TIAB] OR "cryosupernatant plasma"[TIAB] OR "homologous plasma"[TIAB] OR "allogeneic plasma"[TIAB] OR "allogenic plasma" $[T I A B]$ OR "allo-genic plasma" [TIAB] OR "universal plasma" $[T I A B]$ OR "clinical plasma" $[$ TIAB] OR "human plasma" $[T I A B]$ OR "pooled plasma"[TIAB] OR "versus plasma"[TIAB] OR FFP[TIAB] OR SDFFP[TIAB] OR F24[TIAB] OR FP24[TIAB] OR PF24[TIAB] OR MBFFP[TIAB] OR MB-FFP[TIAB] OR SD-FFP[TIAB] OR uniplas*[TIAB] OR octaplas*[TIAB] OR lyoplas*[TIAB] OR frischplasma[TIAB] OR "plasma versus" $[T I A B]$ OR "pathogen-inactivated plasma"[TIAB] OR "pathogen-reduced plasma"[TIAB] OR "plasma units"[TIAB] OR "units of plasma"[TIAB] OR "plasma ratio"[TIAB]

\#3 \#1 OR \#2

\#4 surgery[TIAB] OR surgical ${ }^{\star}[T I A B]$ OR presurg ${ }^{\star}[T I A B]$ OR postsurg ${ }^{\star}[T I A B]$ OR operate* $[T I A B]$ OR operation[TIAB] OR operations[TIAB] OR operating $[T I A B]$ OR preoperat ${ }^{\star}[T I A B]$ OR perioperat ${ }^{\star}[T I A B]$ OR postoperat ${ }^{\star}[T I A B]$ OR transplant $[T I A B]$ OR transplants[TIAB] OR transplanted $[T I A B]$ OR transplanting $[T I A B]$ OR transplantation* $[T I A B]$

\#5 \#3 AND \#4

\#6 (random* OR blind* OR "control group" OR placebo* OR controlled OR groups OR trial OR systematic[sb]) AND (publisher[sb] OR inprocess[sb] OR pubmednotmedline[sb])

\#7 \#5 AND \#6

\section{Appendix 8. Transfusion Evidence Library search strategy}

Subject Area: Plasma/FFP

\section{Appendix 9. LILACS search strategy}

tw:((plasma OR FFP OR SDFFP OR MBFFP OR F24 OR FP24 OR PF24 OR MB-FFP OR SD-FFP OR octaplas OR uniplas OR lyoplas) AND (surgery OR surgical OR surgically OR presurgery OR presurgical OR postsurgery OR operated OR operation OR operations OR operating OR preoperation OR preoperating OR perioperation OR perioperated OR postoperated OR postoperating OR transplant OR transplants OR transplanted OR transplanting OR transplantation OR transplantations)) AND (instance:"regional") AND (db:("LILACS") AND type_of_study: ("clinical_trials" OR "systematic_reviews"))

\section{Appendix 10. Web of Science: CPCI-S search strategy}

\#1 TS=(plasma NEAR/5 (transfus* OR prophyla* OR fresh* OR frozen OR freez* OR prefrozen OR prefreez* OR thaw* OR prethaw* OR liquid OR infus ${ }^{\star}$ OR treatment ${ }^{\star}$ OR therap* OR administ* OR donor* OR donat* OR pasteurized OR pasteurised OR methylene OR solvent OR detergent OR cryoprecipitate OR supernatant OR cryosupernatant OR pathogen $\left.{ }^{\star}\right)$ )

\#2 TS=((homolog* OR allogen* OR allo-gen* OR universal OR clinical OR human OR pooled OR versus OR unit* OR ratio*) NEAR/1 plasma) \#3 TS=(FFP OR SDFFP OR MBFFP OR F24 OR FP24 OR PF24 OR MB-FFP OR SD-FFP OR uniplas* OR octaplas* OR lyoplas* OR frischplasma OR plasma versus)

\#4 \#1 OR \#2 OR \#3

\#5 TS=(surg* OR presurg* OR postsurg* OR operat* OR preoperat* OR perioperat* OR postoperat* OR transplant*) \#6 \#4 AND \#5

\#7 TS=(systematic* OR random* OR blind* OR trial* OR controlled OR control group* OR groups)

\#8 \#6 AND \#7

\section{Appendix 11. ClinicalTrials.gov search strategy}

Search Terms: randomized OR randomised OR randomly

Interventions: Biological: Plasma OR FFP OR SDFFP OR MBFFP OR F24 OR FP24 OR PF24 OR MB-FFP OR SD-FFP OR uniplas OR octaplas OR lyoplas OR plasma transfusion OR prophylactic plasma OR fresh plasma OR frozen plasma OR liquid plasma OR

Search Terms: randomized OR randomised OR randomly

Interventions: universal plasma OR human plasma OR units plasma OR plasma units OR pathogen-reduced plasma 


\section{Appendix 12. WHO ICTRP search strategy}

Recruitment: ALL

Intervention: FFP OR SDFFP OR MBFFP OR F24 OR FP24 OR PF24 OR MB-FFP OR SD-FFP OR plasma transfusion OR prophylactic plasma OR fresh plasma OR frozen plasma OR uniplas OR octaplas OR lyoplas OR universal plasma OR human plasma OR plasma units OR thawed plasma OR liquid plasma OR pathogen-reduced plasma OR pathogen-inactivated plasma

OR

randomized AND plasma transfusion OR randomized AND FFP OR randomized AND fresh plasma OR randomized AND frozen plasma OR randomized AND freeze dried plasma OR randomized AND prophylactic AND plasma OR randomized AND prophylaxis AND plasma OR randomized AND octaplas OR randomized AND uniplas OR randomized AND lyoplas OR randomized AND clinical plasma OR randomized AND universal plasma OR randomized AND human plasma OR randomized AND plasma units OR randomized AND units plasma OR randomized AND biological plasma OR randomized AND thawed plasma OR randomized AND liquid plasma OR randomized AND pathogenreduced plasma OR randomized AND pathogen-inactivated plasma

OR

randomised AND plasma transfusion OR randomised AND FFP OR randomised AND fresh plasma OR randomised AND frozen plasma OR randomised AND freeze dried plasma OR randomised AND prophylactic AND plasma OR randomised AND prophylaxis AND plasma OR randomised AND octaplas OR randomised AND uniplas OR randomised AND lyoplas OR randomised AND clinical plasma OR randomised AND universal plasma OR randomised AND human plasma OR randomised AND plasma units OR randomised AND units plasma OR randomised AND biological plasma OR randomised AND thawed plasma OR randomised AND liquid plasma OR randomised AND pathogenreduced plasma OR randomised AND pathogen-inactivated plasma

OR

randomly AND plasma transfusion OR randomly AND FFP OR randomly AND fresh plasma OR randomly AND frozen plasma OR randomly AND freeze dried plasma OR randomly AND prophylactic AND plasma OR randomly AND prophylaxis AND plasma OR randomly AND octaplas OR randomly AND uniplas OR randomly AND lyoplas OR randomly AND clinical plasma OR randomly AND universal plasma OR randomly AND human plasma OR randomly AND plasma units OR randomly AND units plasma OR randomly AND biological plasma OR randomly AND thawed plasma OR randomly AND liquid plasma OR randomly AND pathogen-reduced plasma OR randomised AND pathogen-inactivated plasma

\section{CONTRIBUTIONS OF AUTHORS}

- Jonathan Huber: content expert. Undertook screening and selection of trials, data extraction, assessment of risk of bias, analysis of results, and development, drafting, and preparation of the protocol and the final report.

- Simon Stanworth: content expert. Supported development and preparation of the protocol and the final report.

- Carolyn Doree: contributed to protocol development and served as search specialist.

- Patricia Fortin: extracted data.

- Marialena Trivella: contributed to protocol development, served as statistical expert, and supported preparation of the final report.

- Susan Brunskill: contributed to protocol development and served as methodologist.

- Sally Hopewell: contributed to protocol development and served as methodological expert.

- Kirstin Wilkinson: content expert. Supported development of the protocol and the final report. Undertook screening and selection of trials and data extraction.

- Lise Estcourt: content expert. Undertook screening and selection of trials, data extraction, assessment of risk of bias, and analysis of results, and developed and prepared the protocol and the final report.

\section{DECLARATIONS OF INTEREST}

- Jonathan Huber: none to declare.

- Simon Stanworth: none to declare.

- Carolyn Doree: none to declare.

- Patricia Fortin: funded in part by the NIHR Cochrane Programme Grant - Safe and Appropriate Use of Blood Components.

- Marialena Trivella: funded in part by the NIHR Cochrane Programme Grant - Safe and Appropriate Use of Blood Components.

- Susan Brunskill: none to declare.

- Sally Hopewell: funded in part by the NIHR Cochrane Programme Grant - Safe and Appropriate Use of Blood Components.

- Kirstin Wilkinson: none to declare.

- Lise Estcourt: funded in part by the NIHR Cochrane Programme Grant - Safe and Appropriate Use of Blood Components. 


\section{SOURCES OF SUPPORT}

\section{Internal sources}

- NHS Blood and Transplant, Research and Development, UK.

To fund the work of the Systematic Review Initiative (SRI)

\section{External sources}

- National Institute for Health Research (NIHR) Cochrane Programme Grant, UK, Other.

To provide funding for systematic reviewers and methodological support from the Centre for Statistics in Medicine, Oxford

\section{DIFFERENCES BETWEEN PROTOCOL AND REVIEW}

Given that we have used a broad definition of the term 'surgery' (based on the definition created by the American College of Surgeons in 2007 (ACS 2007), together with the classification of procedures from the International Classification of Diseases, Ninth Revision, Clinical Modification (ICD9-CM 2011)), we amended the review title to include non-cardiac surgery or invasive procedures, to reflect more accurately the scope of this review (Huber 2017).

We excluded studies that reported only on central line insertion because these studies are included in Hall 2016.

We amended the typographical time scale error for measurements of surgical adverse event outcomes as described in the protocol section 'Summary of findings table'. We measured this outcome within 30 days, consistent with our protocol description of this outcome in the methods section.

\section{IN DEX TERMS}

\section{Medical Subject Headings (MeSH)}

${ }^{*}$ Surgical Procedures, Operative; Anticoagulants [adverse effects] [*therapeutic use]; Blood Component Transfusion [ ${ }^{\star}$ methods]; Hemorrhage [ ${ }^{*}$ prevention \& control]; Hemostatics [therapeutic use]; Plasma; Preoperative Care; Randomized Controlled Trials as Topic; Thrombelastography

\section{MeSH check words}

Humans 